\title{
A CATALOG OF NEAR-INFRARED SPECTRA FROM TYPE Ia SUPERNOVAE
}

\author{
G. H. Marion ${ }^{1}$, P. Höflich ${ }^{2}$, C. L. Gerardy ${ }^{2}$, W. D. Vacca ${ }^{3}$, J. C. Wheeler ${ }^{1}$, and E. L. Robinson ${ }^{1}$ \\ ${ }^{1}$ Astronomy Department, University of Texas at Austin, Austin, TX 78712, USA \\ ${ }^{2}$ Physics Department, Florida State University, Tallahassee, FL 32306, USA \\ ${ }^{3}$ SOFIA-USRA, NASA Ames Research Center, MS N211-3, Moffett Field, CA 94035-1000, USA \\ Received 2008 May 22; accepted 2009 June 21; published 2009 July 20
}

\begin{abstract}
We present 41 near-infrared (NIR, 0.7-2.5 $\mu \mathrm{m}$ ) spectra from normal Type Ia supernovae (SNe Ia) obtained at epochs ranging from 14 days before to 75 days with respect to the maximum light date in the $V$ band. All data were obtained at the Infrared Telescope Facility using the SpeX instrument. We identify many spectral features, measure the Doppler velocities, and discuss the chemical distribution of explosion products in SNe Ia. We describe procedures for smoothing data, fitting continua, and measuring absorption features to ensure consistency for measurement and analysis. This sample provides the first opportunity to examine and compare a large number of SNe Ia in this wavelength region. NIR data are a rich source of information about explosion products whose signatures are blended or obscured in other spectral regions and NIR observations probe a greater radial depth than optical wavelengths. We analyze similarities and differences in the spectra and we show that the progressive development of spectral features for normal SNe Ia in the NIR is consistent with time. We confirm the presence of O I, Mg II, Ca II, Si II, Fe II, and Co II in these SNe. Possible identifications are made for S I, Si III, Mn II, and Fe III. There is no evidence in these data for $\mathrm{H}$ I, He I, C I, or C II. As the explosion products expand and cool, progressively deeper layers are revealed. Thus, a time sequence of spectra examines the chemical structure and provides direct evidence of the physical properties of SNe Ia from the outer layers to deep inside the SN. Measured Doppler velocities indicate that burning products in SNe Ia are distributed in distinct layers with no large-scale mixing. Carbon is not detected in these data, in agreement with previous results with NIR data establishing very low limits on carbon abundance in SNe Ia. Carbon burning products, $\mathrm{O}$ and $\mathrm{Mg}$, are plentiful in the outer layers suggesting that the entire progenitor is burned in the explosion. The data provide a resource for investigations of cross-correlations with other data libraries that may further constrain SN Ia physics and improve the effectiveness of SNe Ia as cosmological distance indicators.
\end{abstract}

Key words: cosmology: observations - infrared: general - line: identification - supernovae: general

Online-only material: color figures

\section{INTRODUCTION}

This sample of 41 near-infrared (NIR, 0.7-2.5 $\mu \mathrm{m}$ ) spectra from Type Ia supernovae ( $\mathrm{SNe}$ Ia) provides the first opportunity to study NIR spectral evolution in a large number of SNe Ia.

$\mathrm{SNe}$ Ia are intrinsically interesting because they are very powerful explosions $\left(\approx 10^{51} \mathrm{erg}\right)$ involving extreme and exotic physics. SNe Ia are also key contributors to the chemical evolution of the universe. In recent years, the relative uniformity and high luminosity of SNe Ia have made them important as "standard candles" used to make distance estimates at large redshifts. SNe Ia data are a valuable tool for measuring many cosmological parameters. The fact that distant SNe Ia appear slightly dimmer than expected for a flat, coasting universe can be used to show that the expansion rate of the universe is accelerating (Garnavich et al. 1998; Riess et al. 1998; Schmidt et al. 1998; Perlmutter et al. 1999). This important result is combined with information from studies of the cosmic microwave background and large-scale structure to suggest the existence of a "dark energy" in the universe.

$\mathrm{SNe}$ Ia are not precisely standard candles, and variability in SN Ia observables introduces uncertainties that limit their effectiveness as distance indicators. The intrinsic dispersion in the peak brightness of SNe Ia events can be constrained to about 0.2 mag by calibrating the measured luminosity using corrections determined by the shape of the light curve (Phillips 1993; Riess et al. 1995; Hamuy et al. 1996; Jha et al. 2007; Guy et al. 2007; Conley et al. 2008). This accuracy is sufficient for some cosmological analysis, but in order to achieve the level of high-precision cosmology required for dark energy measurements the dispersion must be reduced by an order of magnitude (Weller \& Albrecht 2002; Kowalski et al. 2008).

The NIR is a productive source of information about lightand intermediate-mass elements such as $\mathrm{He}, \mathrm{C}, \mathrm{O}, \mathrm{Mg}$, and $\mathrm{Mn}$ that are undetectable or obscured by line blending at other wavelengths. The progenitor of an SN Ia is expected to be a carbon/oxygen white dwarf star (C/O WD), so that after the explosion a region where $\mathrm{C}$ and $\mathrm{O}$ are detected together is composed of primordial material that has not experienced nuclear burning. The extent of nuclear burning in the SN will consequently be defined by a boundary between a region in which $\mathrm{C}$ or $\mathrm{O}$ or both have been consumed and a region that contains both $\mathrm{C}$ and $\mathrm{O}$. The abundance of $\mathrm{Mn}$ is temperature dependent so Mn can be used to probe the burning temperature in regions of incomplete silicon burning (Höflich et al. 1998, 2002). $\mathrm{Si}, \mathrm{S}, \mathrm{Ca}, \mathrm{Fe}$, and $\mathrm{Co}$ are burning products that also produce strong lines in the NIR, making data from this wavelength region a rich source of information about the physical characteristics of SNe Ia.

Many of these important explosion products are revealed by spectral observations of SNe Ia made near or before the date of maximum brightness in the $V$ band $\left(V_{\max }\right)$. Our sample is an excellent resource for investigation of $\mathrm{SNe}$ Ia because it includes 11 spectra obtained before $V_{\max }$, and 20 spectra obtained within 1 week of maximum brightness.

SNe expand and cool during the first weeks after the explosion which is coincident with the time period covered by these observations. As the envelope thins and the dominance of 
Thomson scattering in the continuum diminishes, progressively deeper regions of the $\mathrm{SN}$ are revealed. The data show that NIR spectral features evolve consistently for normal SNe Ia, so a time sequence of spectra can be used to trace the chemical structure from the outer layers toward the center. NIR observations are particularly effective for this purpose because the optical depth is less in the NIR than at shorter wavelengths so that a greater radial depth can be probed with each spectrum.

With a sample of this size, NIR data begin to realize their potential to further constrain estimates for the intrinsic brightness of individual SNe Ia by providing direct evidence of their physical properties. This is an essential part of the interaction between theoretical modeling of SN physics and observational analysis. NIR spectra help define the chemical structure and constrain abundances of some burning products in $\mathrm{SNe}$ Ia.

Relatively few NIR spectra are found in the literature and they were obtained from even fewer individual SNe Ia. Six spectra from -8 to +8 days were obtained from SN1994D in narrow wavelength bands (Meikle et al. 1996). An excellent time sequence for SN 1999ee from -9 to +42 days was obtained by Hamuy et al. (2002), but without comparison with other objects, the data provide no information about the typical behavior for SNe Ia. A fine set of NIR spectra was obtained by Gerardy from SNe Ia SN 1999by between -4 and +28 days (Höflich et al. 2002), but this object was significantly subluminous and does not contribute to the discussion of normal behavior in SNe Ia. Our program previously published 13 spectra from 12 objects (spectra that are included in the current sample) that provided clues for many of the conclusions derived from the larger sample (Marion et al. 2003).

The spectra in this sample also provide an opportunity to search for secondary relationships between NIR observables and other data libraries that may further constrain SN Ia physics and improve the effectiveness of SNe Ia as cosmological distance indicators. For example, this sample may provide a link to accurate " $K$ corrections" for photometric observations in the $J, H$, and $K$ bands.

Details of the data acquisition and reduction procedures are presented in Section 2, including the use of the Fourier transform (FT) to smooth the data. The spectra are presented in Section 3. We identify many spectral features, measure the Doppler velocities for individual ions, and discuss the radial distribution of explosion products. In Section 4, we discuss some of the physical implications of our results. We examine the evolution of spectral features and compare spectra obtained at similar ages noting the similarities and differences. Generally accepted characteristics of the physical properties and behaviors of SNe Ia are reviewed in Section 5 to provide a basis for analysis of the spectra. Section 6 summarizes the results.

The Appendix provides a detailed discussion of individual features in the spectra. Included with the appendix are Tables 5 and 6 that have estimated line strengths of the strongest lines for many of the ions expected to be present in NIR spectra from SNe Ia. The line-strength estimates are separately computed at two temperatures: $5000 \mathrm{~K}$, which is expected to be reasonable for extended line-forming regions, and $10,000 \mathrm{~K}$, which is expected where lines are formed closer to the photosphere.

\section{ACQUISITION AND REDUCTION OF DATA}

We obtained low- and medium-resolution NIR spectra from $\mathrm{SNe}$ Ia using the $3.0 \mathrm{~m}$ telescope at the NASA Infrared
Telescope Facility (IRTF) with the SpeX medium-resolution spectrograph (Rayner et al. 2003). The SpeX instrument provides single exposure coverage of the wavelength region from 0.8 to $2.5 \mu \mathrm{m}$. For SpeX observations using a grating and prism cross-dispersers (SXD mode), the average instrumental spectral resolution $\left(R_{\lambda}=\lambda / \Delta \lambda\right)$ is $750-2000$ and with a single prism (LRS mode) the resolution is 120-300. Most of our data were obtained using one of the three settings: the 0.'5 slit in LRS $\left(R_{\lambda} \approx 200\right.$, which makes the velocity resolution $\left.R_{\mathrm{vel}}=(\Delta \lambda \times c) / \lambda \approx 750 \mathrm{~km} \mathrm{~s}^{-1}\right)$, the $0^{\prime} .8$ slit in SXD $\left(R_{\lambda} \approx 750, R_{\mathrm{vel}} \approx 400 \mathrm{~km} \mathrm{~s}^{-1}\right)$, and the $0^{\prime} .5$ slit in SXD $\left(R_{\lambda} \approx 1200, R_{\mathrm{vel}} \approx 250 \mathrm{~km} \mathrm{~s}^{-1}\right)$. The velocity resolution estimates are calculated for $1.0 \mu \mathrm{m}$ and assume that for the LRS data we can accurately interpolate midway between data points (see Tables 2 and 3). This resolution in velocity space is sufficient because the features we want to investigate have widths of several thousand $\mathrm{km} \mathrm{s}^{-1}$. The LRS observing mode can extend coverage at the blue end of the NIR to about $0.65 \mu \mathrm{m}$. SpeX also contains an infrared slit-viewer/guider covering a $60 \times$ 60 arcsec field of view at 0.12 arcsec pixel ${ }^{-1}$. The detectors are a Raytheon $1024 \times 1024 \mathrm{InSb}$ array in the spectrograph and a Raytheon $512 \times 512$ InSb array in the infrared slit-viewer.

For most observations, the slit was aligned to the parallactic angle at the beginning of the observing sequence. In a few cases, the proximity of the $\mathrm{SN}$ to the host galaxy made it impossible to achieve the optimal parallactic alignment. However, most of our observations were made at less than 1.5 airmasses and the features in which we are interested are near the guiding wavelength, so the errors due to atmospheric refraction are not significant. We do not attempt to compare line strengths and continuum levels with a precision that would be affected by light losses due to misalignment of the slit.

For bright targets, the SpeX guider effectively maintained the centroid of the target in the slit by guiding on the spill-over flux from the object in the slit. When the target did not clearly appear outside the slit, we used the SpeX guider in offset mode with another object in the field of the SpeX imager. Another guiding option was the IRTF optical guider and this was used in a few instances.

Saturation was not a concern due to the faintness of our objects, but $\mathrm{OH}$ lines are numerous and highly variable in the NIR. To avoid an increase in background noise due to poor $\mathrm{OH}$ removal, individual exposure times are capped at $150 \mathrm{~s}$. A typical set is limited to 10 exposures for a total of 25 minutes integration time in order to remain within timescales of atmospheric variability. The source is nodded along the slit using an A_B_B_A_A_B_B_A_A_B pattern. Calibration images are obtained from A0V standard stars. The standards were selected to be as near as possible to the time and airmass of the SN. Each observation set also includes calibration images from internal lamps for flat fielding and wavelength calibrations.

The data were reduced using a package of IDL routines specifically designed for the reduction of SpeX data (Spextool v. 3.2; Cushing et al. 2004). These routines perform pair subtraction, flat-fielding, aperture definition, spectral tracing and extraction, residual sky subtraction, host galaxy subtraction, and wavelength calibration for data acquired in both the prism mode and the cross-dispersed mode. Corrections for telluric absorption were performed using the extracted spectrum of an A0V star and a specially designed IDL package developed by Vacca et al. (2003). These routines generate a telluric correction spectrum by comparing the spectrum of an A0V star to a model A0V spectrum that has been scaled to the observed magnitude, 


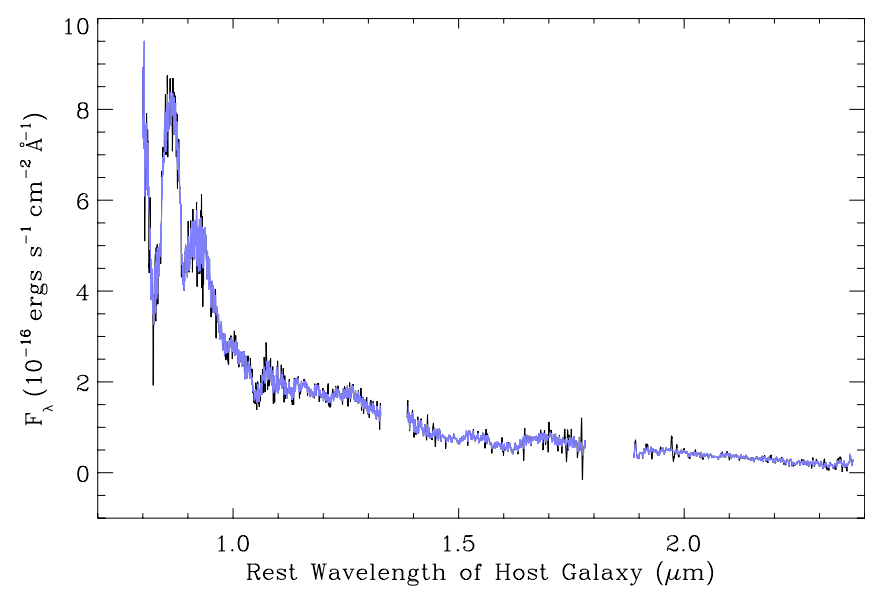

Figure 1. Spectrum from SN 2000dm at +0 day provides an example of our spike elimination routine (see the text in Section 2). The original data are plotted in black and the cleaned spectrum is superimposed in blue (see discussion in Section 2).

(A color version of this figure is available in the online journal.)

smoothed to the observed resolution and shifted to the observed radial velocity. The telluric correction spectrum is then shifted to align the telluric absorption features seen in the SN spectrum and divided into the target spectrum.

Spextool removes problems with background irregularities by defining the aperture width and background level for each subtracted image. The flux level along the slit includes a positive image of the $\mathrm{SN}$ at the A position and a negative image of the $\mathrm{SN}$ at the B position. The background level in the slit is defined by Spextool using a fit through four regions, in two pairs, that bracket each aperture. Typically this is a linear fit, but for noisy spectra a second- or third-order fit is more effective.

Noise levels are calculated and recorded by Spextool for each wavelength bin. Because the data are subject to bad pixels which can skew the combined mean value and the error on the mean, we substitute the median and median absolute deviation (MAD) as robust estimates of these statistics. The MAD is defined as

$$
\mathrm{MAD}=1.4826 \times \operatorname{median}\left(\left|\left(f_{\lambda}-\left\langle f_{\lambda}\right\rangle_{1 / 2}\right)\right|\right),
$$

where $f=$ flux, $\left\langle f_{\lambda}\right\rangle_{1 / 2}$ is the median of the combined values, and 1.4826 is a constant introduced on the assumption of a Gaussian distribution of initial values.

The error on the median at each wavelength is equal to the $1 \sigma$ noise level and equal to the MAD divided by $\sqrt{N}$, where $N$ is the number of spectra that have been combined.

The data have also been cleaned by replacing spikes greater than twice the local noise level (Figure 1). The replacement value from removed spikes is the mean of 40 adjacent data points for SXD and eight adjacent points for LRS spectra.

Observing time scheduled months in advance presents unusual problems when working with transient objects such as SNe. We recognized that the number of potential targets would be improved by more frequent but shorter observing runs. Working with IRTF personnel, we employed a remote observing program to obtain data on systematically scheduled observing dates at a frequency of 8-12 times per year for five years. We obtained high-quality NIR spectra from whatever SNe Ia were available at the scheduled time. In most cases, each target was accessible only once during our observing dates. As a result, we obtained a single epoch or "snapshot" from most of our targets.

\subsection{Using the Fourier Transform Technique to Smooth Spectra}

Using an FT permits us to smooth the data by removing the highest frequency portion of the signal, leaving only the lower frequency components which are the actual information from the target. Since the features we are studying are very broad compared to the spectral resolution, this is an excellent way of reducing the noise to allow more consistent measurements and more effective comparisons between spectra.

The energy flux from SNe Ia drops off rapidly with increasing wavelength, and as a result the signal-to-noise ratio $(\mathrm{S} / \mathrm{N})$ in our data diminishes at longer wavelengths. Due to this different quality of the data, we use different parameters for the FT smoothing in different parts of the spectra. SXD spectra contain $\approx 3900$ data points covering the wavelength range $0.8-2.5 \mu \mathrm{m}$. This large number of bins allows us to separate SXD spectra into three regions: $0.7-1.35 \mu \mathrm{m}, 1.35-1.8 \mu \mathrm{m}$, and 1.8-2.5 $\mu \mathrm{m}$. LRS spectra span the same wavelength band with $\approx 540$ data points. We separate LRS spectra into two sections with the division at about $1.35 \mu \mathrm{m}$.

Each region of a spectrum is individually fit with a straight line between the endpoints and normalized to a flat continuum with the continuum equal to zero. Setting both ends of the spectrum to zero eliminates most of the ringing that can be introduced by the transform procedure. After normalization, a fast FT routine is used to change the spectrum to frequency space.

The power spectrum (signal power by frequency) is obtained by taking the square of the absolute value of the frequency spectrum. A log plot of the power spectrum reveals the noise level forming a horizontal line through the central region of the spectrum. The signal power as a function of frequency descends rapidly from a maximum power in the lowest frequencies located at the edge of the spectrum and it crosses the noise level usually within the first 50 frequency bins. Figure 2(a) shows a log plot of the 50 data points (out of 851) in the power spectrum derived from the region $0.8-1.35 \mu \mathrm{m}$ in the SXD spectrum obtained from SN 2005am at -4 days. The noise level $(N)$ is taken to be constant with a value equal to the mean of the 100 data points immediately to the right of the region displayed $(50-150) . N$ is marked in the figure by a horizontal dashed line.

We estimate that the slope of the signal for these data intersects the noise level near frequency bin 27 . We represent the signal level $(S)$ for each frequency bin using a straight line in $\log$ space from the $y$-intercept $(\approx-4.0)$ through the intersection point: $(27,-12.5)$. To avoid an abrupt transition from the signal line to the noise at the intersection, we generate a filter using $S /(S+N)$ and multiply the frequency spectrum at each bin by the filter at that bin (Figure 2(b)). We explored using a Wiener filter $\left(S^{2} /\left(N^{2}+S^{2}\right)\right)$ and found no measurable difference in the resulting smoothed spectrum. The result of applying the filter to the FT data is shown in Figure 3 with the original frequency spectrum plotted in black and the filtered spectrum is superimposed in red.

As a test of the consistency of our method, we compare the effects of different smoothing parameters on differently shaped absorption features from the same data (SN 2005am at -4d). The smoothing parameter is defined as the number of frequency bins to be included in the signal $(S)$. Figure 4 shows raw data plotted in black with the results of various smoothing parameters superimposed in colors. The top panel displays the feature from $\mathrm{Mg}$ II at $0.9227 \mu \mathrm{m}$ which is asymmetric due to impingement on the red side from emission due to the Ca II IR triplet. The 

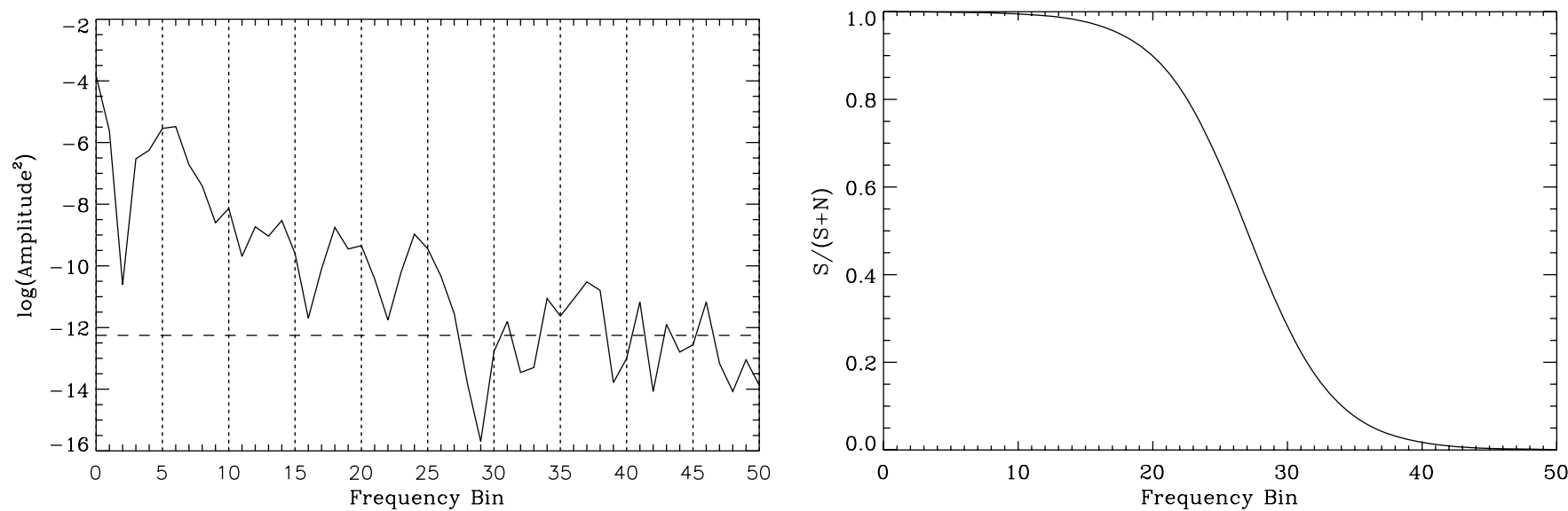

Figure 2. Power spectrum and filter used in our reduction package (see the text in Section 2.1). The left panel is a log plot of the first 50 points (out of 1901) in one half of the power spectrum for the wavelength region 0.80-1.35 $\mu \mathrm{m}$ from the SXD data from SN 2005am at -4 days. The noise level $(N)$ is taken to be a constant equal to the mean of the next 100 points $(50: 150)$. The location of $N$ is indicated in the figure by a horizontal dashed line. The signal $(S)$ descends from near -4 on the left edge and intersects the noise level at point 27. The right panel shows the filter produced by the formula: $S /(S+N)$ (see discussion in Section 2.1).

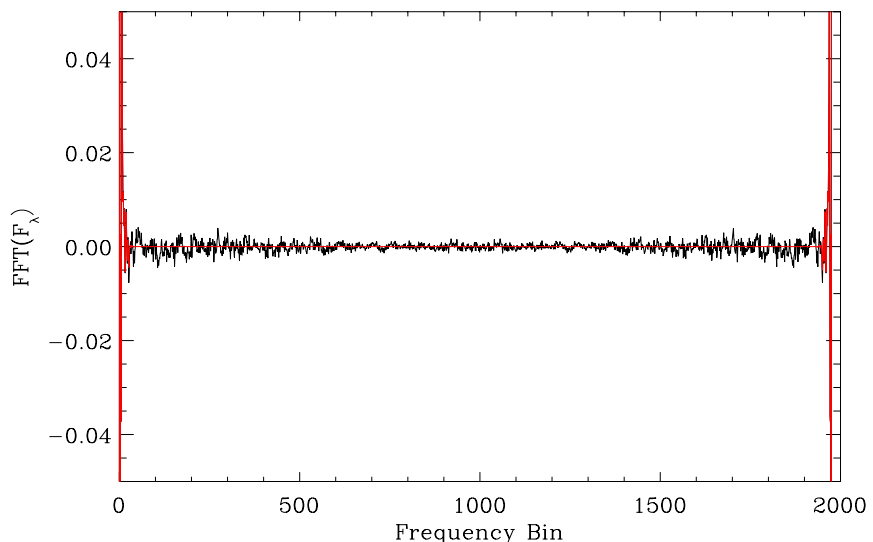

Figure 3. FT for the wavelength region $0.80-1.35 \mu \mathrm{m}$ from SN 2005am at -4 days (see the text in Section 2.1). The initial frequency spectrum is plotted in black. The same spectrum after processing with the filter as described in the text is superimposed in red (see discussion in Section 2.1).

(A color version of this figure is available in the online journal.)

bottom panel shows the feature from the $\mathrm{Mg}$ II $1.0927 \mu \mathrm{m}$ line which is more nearly symmetrical.

The FT smoothed spectrum, derived according to our standard procedure described above and using 27 bins, is plotted in black. We reduce the number of bins until the smoothing obviously compromises the positions of the absorption minima. Different colors are used in the figure to represent the spectrum smoothed using different parameters. Table 1 gives the measured velocities with the different smoothing parameters. The table shows that a very large change in the smoothing parameter, beyond any reasonable choice made by the user, is required to change the measured velocity by more than $200 \mathrm{~km} \mathrm{~s}^{-1}$.

When the smoothing parameter is increased, the change in measured velocity is very small. The primary effect of too large a value of the parameter is that local noise may be included in the smoothed spectrum. However, the features we are measuring are much larger than any local spikes. Consequently, when there were ambiguities in the choice of smoothing parameters, we were careful to avoid choosing smaller values. Interpretations made by individual users may vary the total number of bins selected by 3-5 in the smaller direction and 5-10 in the greater direction, but the figure and table clearly show that differences on that scale have no significant effect on the results. An ex-
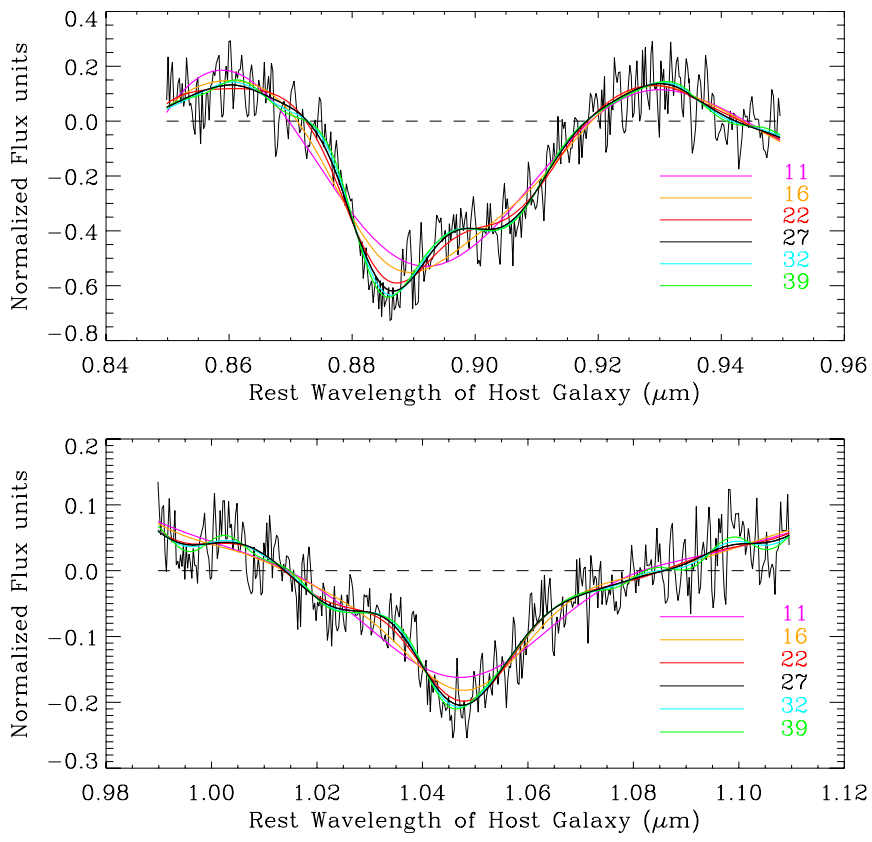

Figure 4. Two absorption features from the high S/N SXD spectrum of SN 2005am at $-4 \mathrm{~d}$ demonstrate that the FT smoothing technique is robust and the measured velocities do not change significantly due to fine interpretations of the smoothing parameter by the user (see the text in Section 2.1 and Table 1). The raw data are black and the smoothed spectrum in black is the FT spectrum as with a smoothing parameter of 27 as shown in Figure 2(a). The spectra produced by other smoothing parameters are plotted in different colors (see discussion in Section 2.1). On this scale, the distance between ticks on the wavelength axis $(0.005 \mu \mathrm{m})$ is about $1500 \mathrm{~km} \mathrm{~s}^{-1}$.

(A color version of this figure is available in the online journal.)

aggerated change on the order of $40 \%$ (from 27 to 16 bins) is far beyond the uncertainty in our parameter choices and it only moves the measured velocity by $100 \mathrm{~km} \mathrm{~s}^{-1}$ for the more symmetrical feature in the bottom panel of Figure 4 and $900 \mathrm{~km} \mathrm{~s}^{-1}$ for the less symmetrical feature in the top panel of Figure 4.

The amount that the spectral resolution is degraded by smoothing depends on the original resolution and the amount of smoothing required. In the example here with the data from SN 2005am at $-4 \mathrm{~d}$ and a smoothing parameter of 27 , the instrumental resolution of $\approx 250 \mathrm{~km} \mathrm{~s}^{-1}$ is reduced to $\approx 1500 \mathrm{~km} \mathrm{~s}^{-1}$, but the measured location of the absorption minimum only changes by $200-300 \mathrm{~km} \mathrm{~s}^{-1}$. 
Table 1

Comparing the Effects of Fourier Transform Smoothing Parameters

\begin{tabular}{lcccccc}
\hline \hline $\begin{array}{l}\text { Smoothing } \\
\text { Parameter }\end{array}$ & $\begin{array}{c}\text { Minimum }(\mu \mathrm{m}) \\
\lambda_{\text {rest }}=1.0927 \mu \mathrm{m}\end{array}$ & $\begin{array}{c}\text { Velocity } \\
\left(\mathrm{km} \mathrm{s}^{-1}\right)\end{array}$ & $\begin{array}{c}\text { Line Depth } \\
\text { Normalized }\end{array}$ & $\begin{array}{c}\text { Minimum }(\mu \mathrm{m}) \\
\lambda_{\text {rest }}=0.9227 \mu \mathrm{m}\end{array}$ & $\begin{array}{c}\text { Velocity } \\
\left(\mathrm{km} \mathrm{s}^{-1}\right)\end{array}$ & $\begin{array}{c}\text { Line Depth } \\
\text { Normalized }\end{array}$ \\
\hline 39 & 1.0463 & 12,700 & 1.00 & 0.8859 & 11,900 & 1.00 \\
32 & 1.0467 & 12,500 & 0.99 & 0.8864 & 11,800 & 0.99 \\
27 & 1.0474 & 12,400 & 0.97 & 0.8866 & 11,700 & 0.96 \\
22 & 1.0479 & 12,300 & 0.95 & 0.8873 & 11,500 & 0.82 \\
16 & 1.0479 & 12,300 & 0.87 & 0.8894 & 10,800 & 0.86 \\
11 & 1.0471 & 12,500 & 0.78 & 0.8921 & 9,900 & 0.82 \\
\hline
\end{tabular}

Note. These data are plotted in Figure 4 and discussed in Section 2.1.

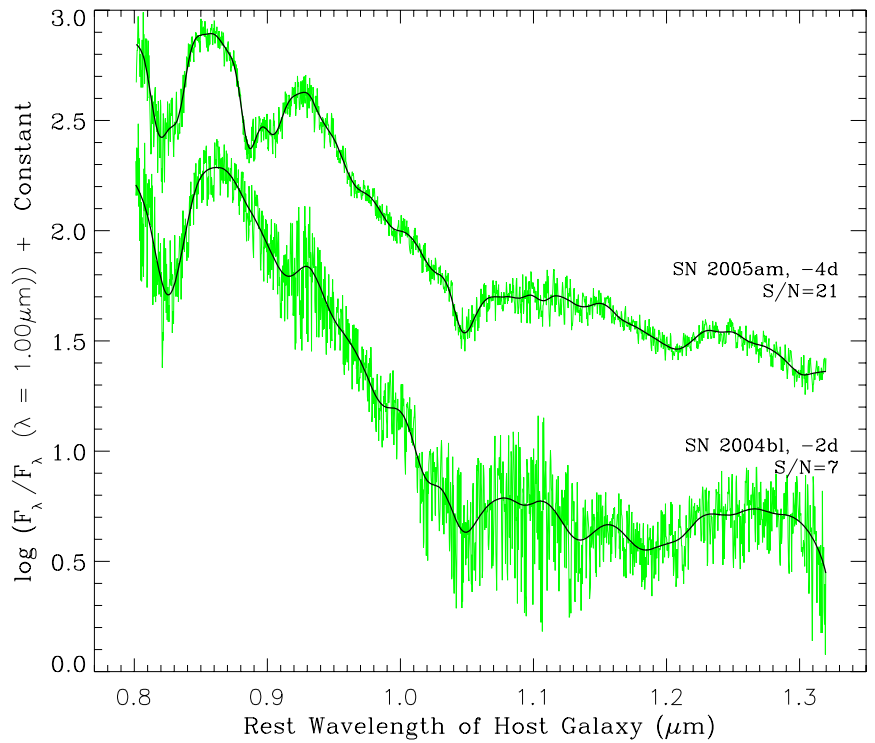

Figure 5. Two spectra with different $\mathrm{S} / \mathrm{N}$ show how the use of the FT smoothed spectra facilitates understanding of the overall spectral shape and identification of the precise locations of maxima and minima (see the text in Section 2.1). The raw data are plotted in green and the FT smoothed spectra are superimposed in black (see discussion in Section 2.1).

(A color version of this figure is available in the online journal.)

The reverse FT of the filtered frequency spectrum returns a smoothed data spectrum that greatly improves our ability to interpret the features. The top spectrum in Figure 5 shows the raw data from a relatively high-quality spectrum obtained from SN 2005am at $-4 \mathrm{~d}$ plotted in green, with the smoothed spectrum plotted in black over the original. The lower spectrum in the figure shows data from a noisier spectrum, SN 2004bl at $-2 \mathrm{~d}$, and again the smoothed spectrum is plotted in black over the original data. The figure illustrates the effectiveness of this technique to facilitate inspection and comparison of spectra in the sample.

\subsection{Fitting a Local Continuum}

The energy flux from SNe Ia decreases rapidly with increasing wavelength. Within this steeply sloping continuum, the wavelength of minimum flux may not correspond to the wavelength of the maximum departure from the continuum. For absorption features, this effect is not significant where the continuum is relatively flat, as in the optical region of the Si II line at $6355 \mu \mathrm{m}$, but it can potentially affect features in the NIR by $\approx 0.005 \mu \mathrm{m}$ or $\approx 1500 \mathrm{~km} \mathrm{~s}^{-1}$. To remove this influence, we estimate the location of the continuum in the region of each absorption feature and normalize the spectrum to a flat continuum before identifying the wavelength of absorption minimum.

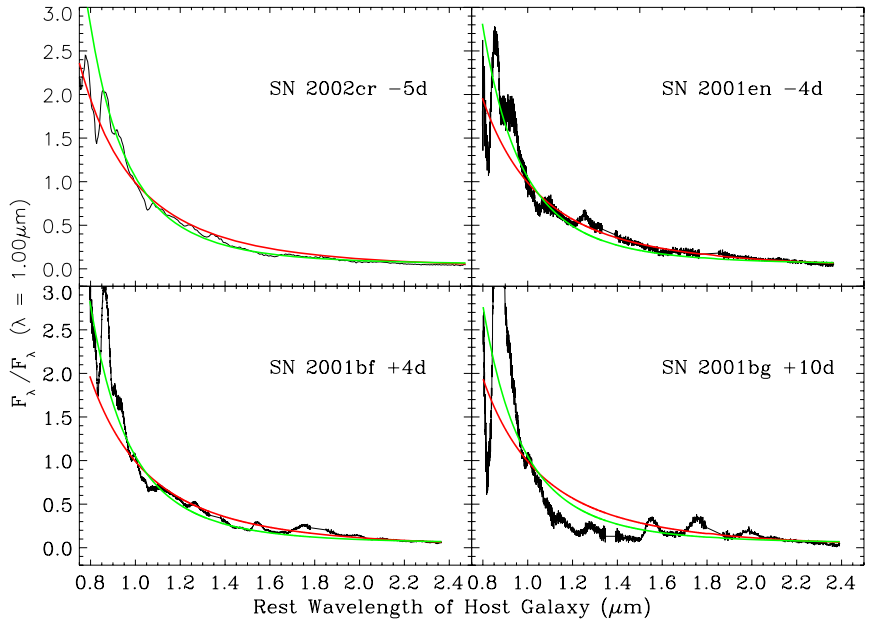

Figure 6. Raw data from four spectra showing possible fits to the continua (see the text in Section 2.2). The top left spectrum is LRS, the others are SXD. The red line is $a-3.0$ power law and the green line is a -4.5 power law. It is clear that a single power law does not fit any of the spectra (see discussion in Section 2.2).

(A color version of this figure is available in the online journal.)

Line-forming regions in $\mathrm{SNe}$ Ia are extended in velocity space (Section 5.3). Thus, a full analysis requires measurement of the full range of Doppler velocities between the absorption minimum and the detection limit of the blue wings for each feature. Due to uncertainties introduced by the sloping continuum, this region can be more accurately measured by normalizing the data to a level continuum.

Figure 6 illustrates the difficulty of fitting a continuum to $\mathrm{SNe}$ Ia spectra over the wavelength region $0.8-2.5 \mu \mathrm{m}$. Four spectra from different epochs are displayed with estimated continua superimposed in red $(-3.0$ power law) and green $(-4.5$ power law). It is clear that a single power law does not fit any spectrum over the entire region. Although we have been as consistent as possible with our observing and reduction techniques, it is possible that some of the differences in slope and shape between spectra may have been introduced by observation or data processing choices. There are, however, obvious changes in the overall shape of the spectra with time and there appear to be measurable differences in the shapes of spectra from different objects at similar epochs.

For the purpose of comparing features from the same line in different spectra, we abandon our efforts to fit a continuum over a large wavelength range and concentrate on fitting a local continuum in the vicinity of each feature. Figure 7 shows the same spectra as the top two panels in Figure 6 with local continua fit to the spectra in the wavelength regions close to each absorption feature. This technique produces better results 


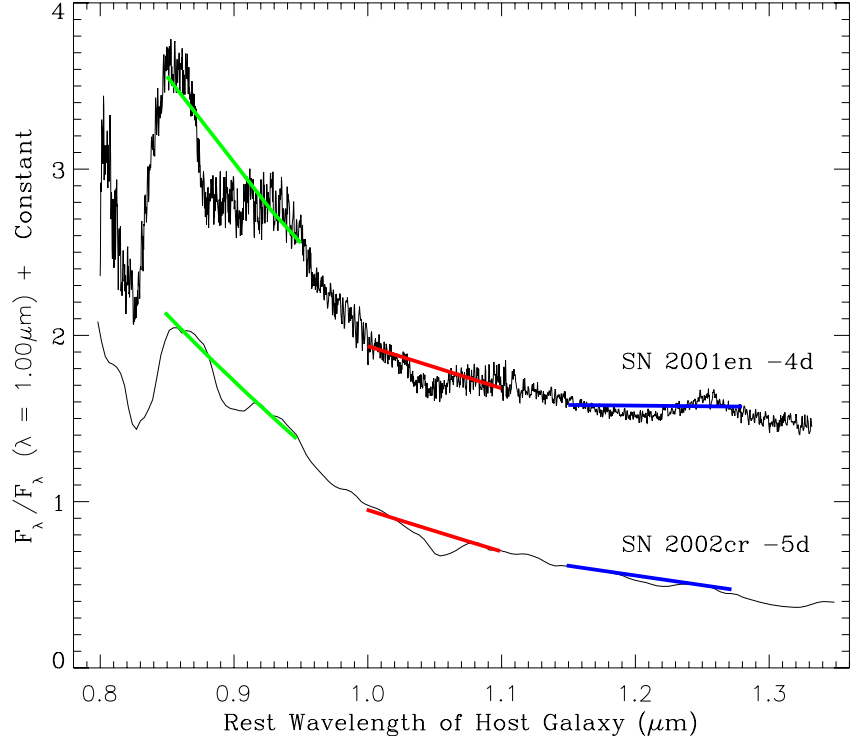

Figure 7. Estimated fits for local continua in the vicinity of absorption features are plotted in the figure in red, green, and blue (see the text in Section 2.2). The top spectrum is a raw data SXD spectrum from SN 2001en at -4 days. On the bottom is a raw LRS spectrum from SN $2002 \mathrm{cr}$ at -5 days. These are the same spectra displayed in the top two panels of Figure 6 and the fitted regions are expanded in Figure 8 (see discussion in Section 2.2).

(A color version of this figure is available in the online journal.)

than fitting a single continuum to the entire spectrum. The fitted regions are expanded in Figure 8.

There remains ambiguity about how to fit the local continua because of differences in the spectra within each region but variations within the limits of our estimates do not significantly affect the results. For example, we assume that the peaks near 0.85 and $0.93 \mu \mathrm{m}$ rise above the continuum as emission components of P Cygni profiles from the very strong Ca II triplet (mean $=0.8579 \mu \mathrm{m})$ and the strong $\mathrm{Mg}$ II line $(0.9227 \mu \mathrm{m})$; however, it is not obvious where the true continuum level lies. Our estimate for the location of the continuum in this region appears as the green line in both panels of Figure 7.

The absorption features in Figure 7 with minima near 1.05 and $1.20 \mu \mathrm{m}$ display different profiles in the two spectra. It is not clear how much of the bump on the red side of the absorption is emission and how much is continuum. The estimated locations of the local continua for these regions are shown as red and blue lines in Figure 7.

Figure 8 expands the spectral regions with locally fit continua from Figure 7 but with the continuum normalized to zero. All figures on the left are from the SXD spectrum of SN 2002en at -4 days. The figures on the right are from an LRS spectrum of SN 2002cr at $-5 \mathrm{~d}$. The top row corresponds to the regions marked in green in Figure 7, the middle row to the red regions, and the bottom row to the blue. The FT smoothed spectrum is superimposed in red on the spectra in all figures. We tested this method by gradually changing the fitted continua until it was clearly beyond a reasonable fit to the data. The largest deviations in the wavelengths of the measured minima due to the manual placement of the continua was found to be $\pm 300 \mathrm{~km} \mathrm{~s}^{-1}$ and for most features the deviations are smaller.

\section{THE SPECTRA}

Our sample consists of 41 NIR spectra from SNe Ia, obtained between 14 days before $(-14 \mathrm{~d})$ and 75 days after $(+75 \mathrm{~d}) V_{\max }$ (Figure 9). All spectra in our sample were obtained at the IRTF
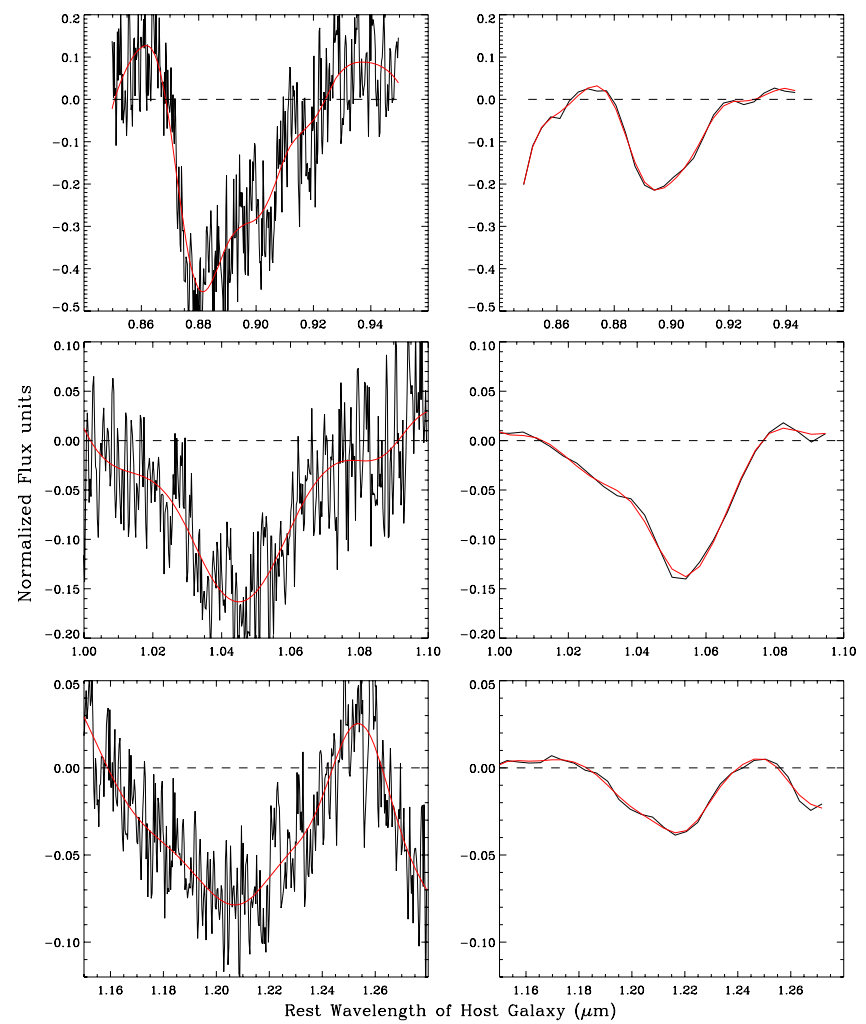

Figure 8. Expanded spectral regions from Figure 7 with the continuum normalized to zero (see the text in Section 2.2). The left column displays SXD data from SN 2001en (-4d) and the right column is LRS data from SN $2002 \mathrm{cr}$ $(-5 \mathrm{~d})$. The top row corresponds to the regions in Figure 7 marked with a green continuum, the middle row is from the red regions, and the bottom row is from the blue regions. The FT smoothed spectrum is superimposed on the raw data in red (see discussion in Section 2.2).

(A color version of this figure is available in the online journal.)

using the SpeX instrument. Observation and reduction practices are discussed in Section 2.

The spectra are listed in Table 2 by epoch with respect to $V_{\max }$ and Table 3 in order of discovery. The Tables include: SN names, estimated ages at observation (with respect to $V_{\max }$ ), the dates of observation, the average instrumental resolution observing mode, the estimated $\mathrm{S} / \mathrm{N}$ for wavelength regions approximately corresponding to $Y, J, H$, and $K$ bands, and the redshift $(z)$ of the host galaxy. The epoch of observation with respect to $V_{\max }$ is estimated by a variety of means and we have attempted to provide as accurate information as possible. More complete light curve data for many of the $\mathrm{SNe}$ in our sample are in preparation and the observation date for each spectrum is included in the tables in order to facilitate comparison when these data become available.

In all figures containing multiple spectra, we arrange the spectra in sequence with the earliest spectrum at the top according to the time of observation with respect to $V_{\max }$. The abscissa is wavelength, in microns, shifted to the rest frame of the host galaxy and the ordinate is $\log$ flux.

Each spectrum has been normalized to 1.0 at $1.0 \mu \mathrm{m}$. The names of the $\mathrm{SNe}$ and their age when observed (in days relative to $V_{\max }$ ) are displayed to the right of each spectrum in the same color as the spectrum. The colors for each spectrum are consistent throughout the colored figures to facilitate identification. The black line superimposed on the original data is the smoothed spectrum obtained by FT as described in Section 2. 


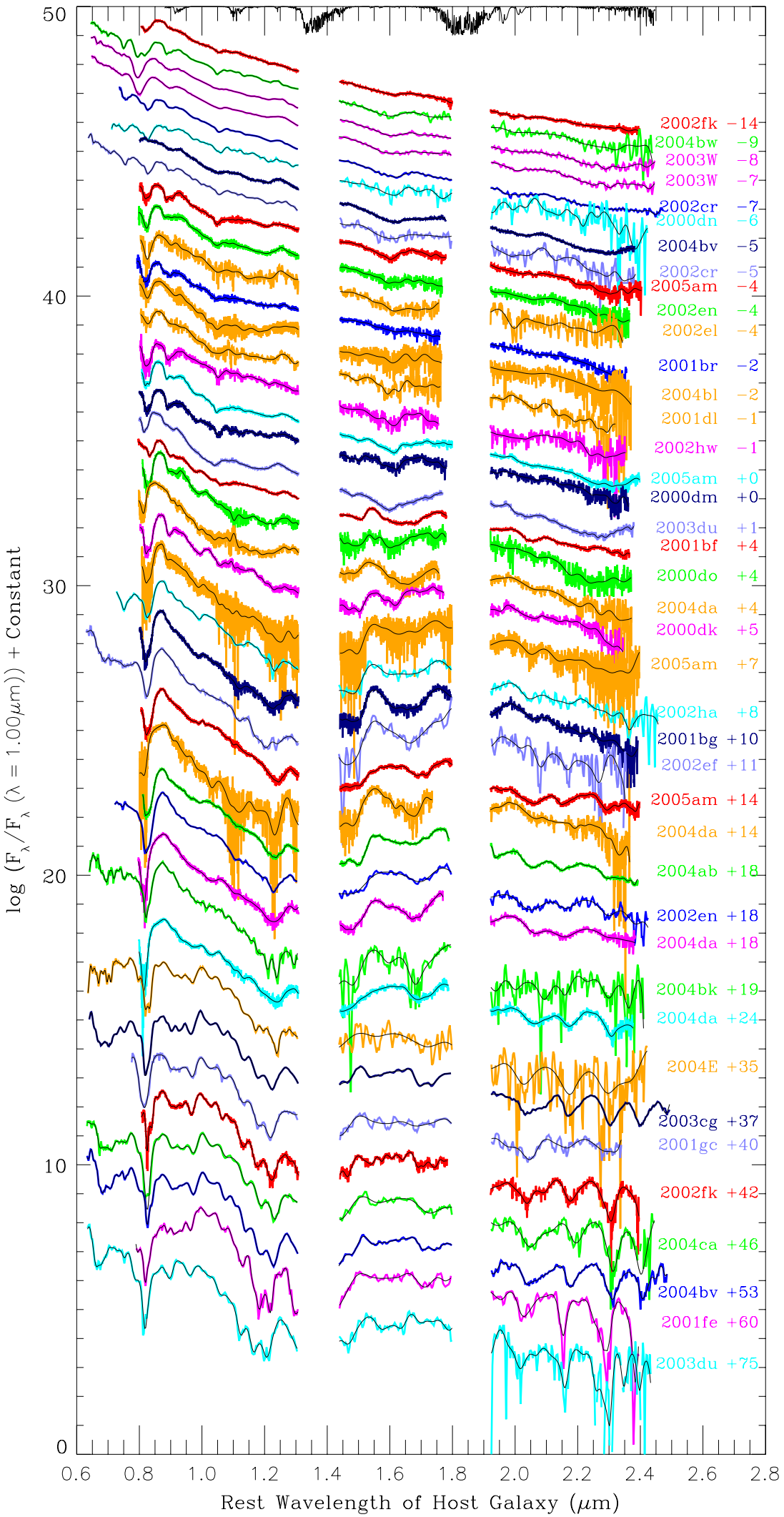

Figure 9. Forty-one spectra from normal SNe Ia obtained between $-14 \mathrm{~d}$ and $+75 \mathrm{~d}$ with respect to $V_{\max }$ (see the text in Section 3 ). The abscissa is wavelength in microns, shifted to the rest frame of the host galaxy and the ordinate is log flux. All spectra have been normalized to 1.0 at $1.0 \mu \mathrm{m}$ and shifted by a constant for clarity. Spectra plotted in yellow have low $\mathrm{S} / \mathrm{N}$ and will be omitted for the analysis of spectral features. Observational and spectral details are provided in Tables 2 and 3. (A color version of this figure is available in the online journal.)

Atmospheric opacity depends strongly on wavelength in the NIR. A transmission spectrum is provided at the top of some figures to help identify regions where opacity reduces the signal.
Complete transparency is at the top axis of the plot and full opacity at one unit down. The transmission spectrum has been shifted using $z=0.02$ which is the approximate mean redshift 
Table 2

List of Spectra by Estimated Epoch Relative to $V_{\max }$

\begin{tabular}{|c|c|c|c|c|c|c|c|c|}
\hline $\begin{array}{c}\text { SN } \\
\text { Name } \\
\end{array}$ & $\begin{array}{c}\text { Est. Epoch } \\
\text { wrt } V_{\max } \\
\end{array}$ & $\begin{array}{c}\text { Obs. } \\
\text { Date (UT) }\end{array}$ & $\begin{array}{l}\text { Avg. } \\
\text { Res. }\end{array}$ & $\begin{array}{c}\mathrm{S} / \mathrm{N} \\
(0.95-1.10 \mu \mathrm{m}) \\
\end{array}$ & $\begin{array}{c}\mathrm{S} / \mathrm{N} \\
(1.10-1.30 \mu \mathrm{m}) \\
\end{array}$ & $\begin{array}{c}\mathrm{S} / \mathrm{N} \\
(1.45-1.70 \mu \mathrm{m}) \\
\end{array}$ & $\begin{array}{c}\mathrm{S} / \mathrm{N} \\
(1.95-2.35 \mu \mathrm{m}) \\
\end{array}$ & $\begin{array}{l}\text { Redshift } \\
\text { Host (z) } \\
\end{array}$ \\
\hline $2002 \mathrm{fk}$ & $-14 d$ & Sep 19.4 & 1200 & 74 & 67 & 47 & 25 & 0.007125 \\
\hline $2004 \mathrm{bw}$ & $-9 \mathrm{~d}$ & May 30.4 & 200 & 74 & 54 & 17 & 12 & 0.021408 \\
\hline $2003 W$ & $-8 \mathrm{~d}$ & Feb 2.4 & 200 & 104 & 29 & 47 & 22 & 0.020071 \\
\hline $2003 \mathrm{~W}$ & $-7 d$ & Feb 3.4 & 200 & 144 & 132 & 41 & 25 & 0.020071 \\
\hline $2002 \mathrm{cr}$ & $-7 d$ & May 8.4 & 200 & 153 & 133 & 77 & 40 & 0.009590 \\
\hline $2000 \mathrm{dn}$ & $-6 d$ & Oct 1.5 & 120 & 37 & 34 & 7 & 3 & 0.032019 \\
\hline $2004 \mathrm{bv}$ & $-5 \mathrm{~d}$ & May 30.5 & 1200 & 91 & 69 & 54 & 37 & 0.010544 \\
\hline $2002 \mathrm{cr}$ & $-5 \mathrm{~d}$ & May 10.3 & 200 & 53 & 37 & 11 & 4 & 0.009590 \\
\hline 2005am & $-4 d$ & Mar 5.3 & 1200 & 20 & 21 & 18 & 12 & 0.007889 \\
\hline 2001en & $-4 d$ & Oct 8.4 & 1200 & 15 & 19 & 10 & 7 & 0.015871 \\
\hline $2002 \mathrm{el}$ & $-4 d$ & Aug 20.5 & 750 & 6 & 7 & 4 & 4 & 0.023300 \\
\hline $2001 \mathrm{br}$ & $-2 d$ & May 22.6 & 750 & 13 & 18 & 9 & 7 & 0.020628 \\
\hline 2004bl & $-2 d$ & May 8.3 & 1200 & 7 & 7 & 4 & 3 & 0.017319 \\
\hline $2001 \mathrm{dl}$ & $-1 d$ & Aug 12.5 & 750 & 9 & 12 & 7 & 5 & 0.020694 \\
\hline $2002 \mathrm{hw}$ & $-1 \mathrm{~d}$ & Nov 14.3 & 1200 & 12 & 12 & 6 & 4 & 0.017535 \\
\hline 2005am & $+0 \mathrm{~d}$ & Mar 9.4 & 1200 & 30 & 32 & 22 & 16 & 0.007889 \\
\hline $2000 \mathrm{dm}$ & $+0 \mathrm{~d}$ & Oct 1.2 & 750 & 15 & 21 & 10 & 9 & 0.015304 \\
\hline $2003 \mathrm{du}$ & $+1 d$ & May 9.3 & 1200 & 47 & 31 & 24 & 15 & 0.016764 \\
\hline $2001 \mathrm{bf}$ & $+4 d$ & May 21.5 & 1200 & 50 & 51 & 36 & 29 & 0.015501 \\
\hline $2000 \mathrm{do}$ & $+4 d$ & Oct 2.2 & 750 & 15 & 10 & 7 & 6 & 0.010864 \\
\hline 2004da & $+4 d$ & Jul 8.5 & 750 & 8 & 8 & 6 & 5 & 0.016308 \\
\hline $2000 \mathrm{dk}$ & $+5 \mathrm{~d}$ & Oct 1.5 & 750 & 19 & 14 & 11 & 7 & 0.017439 \\
\hline $2005 \mathrm{am}$ & $+7 \mathrm{~d}$ & Mar 16.4 & 750 & 3 & 2 & 2 & 3 & 0.007889 \\
\hline $2002 \mathrm{ha}$ & $+8 \mathrm{~d}$ & Nov 14.2 & 200 & 60 & 23 & 13 & 10 & 0.014046 \\
\hline $2001 \mathrm{bg}$ & $+10 \mathrm{~d}$ & May 22.2 & 1200 & 18 & 10 & 9 & 7 & 0.007122 \\
\hline $2002 \mathrm{ef}$ & $+11 d$ & Aug 20.6 & 200 & 22 & 7 & 5 & 2 & 0.023977 \\
\hline 2005am & $+14 d$ & Mar 23.3 & 1200 & 26 & 15 & 19 & 13 & 0.007889 \\
\hline $2004 d a$ & $+14 d$ & Jul 18.4 & 750 & 4 & 2 & 5 & 5 & 0.016308 \\
\hline $2004 \mathrm{ab}$ & $+18 \mathrm{~d}$ & Mar 7.5 & 750 & 41 & 27 & 45 & 36 & 0.005807 \\
\hline 2001en & $+18 \mathrm{~d}$ & Oct 30.3 & 200 & 45 & 21 & 18 & 10 & 0.015871 \\
\hline $2004 d a$ & $+18 \mathrm{~d}$ & Jul 22.5 & 750 & 35 & 15 & 30 & 23 & 0.016308 \\
\hline $2004 \mathrm{bk}$ & $+19 d$ & May 8.5 & 200 & 12 & 8 & 2 & 0 & 0.023036 \\
\hline $2004 \mathrm{da}$ & $+24 d$ & Jul 28.4 & 750 & 18 & 10 & 15 & 10 & 0.016308 \\
\hline 2004E & $+35 \mathrm{~d}$ & Feb 21.5 & 200 & 42 & 13 & 6 & 1 & 0.029807 \\
\hline $2003 \mathrm{cg}$ & $+37 d$ & May 9.2 & 200 & 66 & 56 & 80 & 47 & 0.004130 \\
\hline $2001 \mathrm{gc}$ & $+40 \mathrm{~d}$ & Jan 14.5 & 200 & 28 & 12 & 8 & 6 & 0.019290 \\
\hline $2002 \mathrm{fk}$ & $+42 \mathrm{~d}$ & Nov 14.4 & 750 & 37 & 13 & 15 & 10 & 0.007125 \\
\hline $2004 \mathrm{ca}$ & $+46 \mathrm{~d}$ & Jul 28.6 & 200 & 56 & 24 & 13 & 5 & 0.017806 \\
\hline $2004 \mathrm{bv}$ & $+53 d$ & Jul 28.3 & 200 & 63 & 45 & 38 & 16 & 0.010544 \\
\hline $2001 \mathrm{fe}$ & $+60 \mathrm{~d}$ & Jan 14.6 & 120 & 45 & 15 & 12 & 6 & 0.013539 \\
\hline $2003 \mathrm{du}$ & $+75 \mathrm{~d}$ & Jul 22.3 & 200 & 41 & 7 & 8 & 2 & 0.016764 \\
\hline
\end{tabular}

of the SNe Ia in our sample. The noise levels in the data are very high through regions where the opacity exceeds $20 \%$ $(\approx 1.30-1.42 \mu \mathrm{m}$ and $1.78-1.90 \mu \mathrm{m})$. We have removed the data in these regions from all spectra.

Order transitions in SXD spectra occur near 0.95, 1.12, and $1.45 \mu \mathrm{m}$. Small local discontinuities are possible at these locations where the orders are merged. This problem is more pronounced in the noisier spectra. SXD spectra also have gaps between $\approx 1.35$ and 1.42 where there are no data between orders. The gap locations were selected to correspond to regions of high atmospheric opacity.

Type Ia spectra are characterized by the steadily diminishing flux at longer wavelengths, so the $\mathrm{S} / \mathrm{N}$ degrades with increasing wavelength as the level of the signal approaches background noise levels (Tables 3 and 2).

\subsection{Line Identification by Epoch}

For spectral analysis, we removed seven spectra that have $\mathrm{S} / \mathrm{N}$ less than 10 in the region $1.10-1.30 \mu \mathrm{m}$ from the complete sample (see Table 2). This wavelength region approximately corresponds to the $J$ band and contains many of the most important features under discussion. These seven spectra are displayed using the color yellow in Figure 9. The 34 remaining spectra are presented in Figure 10.

For analysis and line identification, we divide the remaining spectra into three groups selected by epoch and spectral features. The boundaries between these phases is not always distinct and there is some temporal overlap between the phases. We define the groups as: Photospheric Phase (outer layers of the $\mathrm{SN} ; 18$ spectra obtained between $-14 \mathrm{~d}$ and $+5 \mathrm{~d})$, Extended Photospheric Phase (intermediate layers of the SN; 10 spectra between $+4 \mathrm{~d}$ and $+24 \mathrm{~d}$ ), and Transitional Phase (inner region of the $\mathrm{SN}$; seven spectra obtained between $+37 \mathrm{~d}$ and $+75 \mathrm{~d}$ ).

We identify many of the prominent features, measure their Doppler velocities, and provide a synopsis of spectral development in each group. The Appendix provides a more detailed analysis of the spectra and features with possible alternative line identifications and a discussion of each ion present and the physical implications. We display the spectra in log space to assist with the identification of features at longer wavelengths, 
Table 3

List of Spectra in Order of Discovery

\begin{tabular}{|c|c|c|c|c|c|c|c|c|}
\hline $\begin{array}{c}\text { SN } \\
\text { Name }\end{array}$ & $\begin{array}{c}\text { Est. Epoch } \\
\text { wrt } V_{\max }\end{array}$ & $\begin{array}{c}\text { Obs. } \\
\text { Date (UT) }\end{array}$ & $\begin{array}{l}\text { Avg. } \\
\text { Res. }\end{array}$ & $\begin{array}{c}\mathrm{S} / \mathrm{N} \\
(0.95-1.10 \mu \mathrm{m}) \\
\end{array}$ & $\begin{array}{c}\mathrm{S} / \mathrm{N} \\
(1.10-1.30 \mu \mathrm{m}) \\
\end{array}$ & $\begin{array}{c}\mathrm{S} / \mathrm{N} \\
(1.45-1.70 \mu \mathrm{m}) \\
\end{array}$ & $\begin{array}{c}\mathrm{S} / \mathrm{N} \\
(1.95-2.35 \mu \mathrm{m}) \\
\end{array}$ & $\begin{array}{l}\text { Redshift } \\
\text { Host (z) } \\
\end{array}$ \\
\hline $2000 \mathrm{dk}$ & $+5 \mathrm{~d}$ & Oct 1.5 & 750 & 19 & 14 & 11 & 7 & 0.017439 \\
\hline $2000 \mathrm{dm}$ & $+0 \mathrm{~d}$ & Oct 1.2 & 750 & 15 & 21 & 10 & 9 & 0.015304 \\
\hline $2000 \mathrm{dn}$ & $-6 d$ & Oct 1.5 & 120 & 37 & 34 & 7 & 3 & 0.032019 \\
\hline $2000 \mathrm{do}$ & $+4 d$ & Oct 2.2 & 750 & 15 & 10 & 7 & 6 & 0.010864 \\
\hline $2001 \mathrm{bf}$ & $+4 d$ & May 21.5 & 1200 & 50 & 51 & 36 & 29 & 0.015501 \\
\hline $2001 \mathrm{bg}$ & $+10 \mathrm{~d}$ & May 22.2 & 1200 & 18 & 10 & 9 & 7 & 0.007122 \\
\hline $2001 \mathrm{br}$ & $-2 d$ & May 22.6 & 750 & 13 & 18 & 9 & 7 & 0.020628 \\
\hline $2001 \mathrm{dl}$ & $-1 d$ & Aug 12.5 & 750 & 9 & 12 & 7 & 5 & 0.020694 \\
\hline $2001 \mathrm{en}$ & $-4 d$ & Oct 8.4 & 1200 & 15 & 19 & 10 & 7 & 0.015871 \\
\hline 2001en & $+18 d$ & Oct 30.3 & 200 & 45 & 21 & 18 & 10 & 0.015871 \\
\hline $2001 \mathrm{fe}$ & $+60 \mathrm{~d}$ & Jan 14.6 & 120 & 45 & 15 & 12 & 6 & 0.013539 \\
\hline $2001 \mathrm{gc}$ & $+40 \mathrm{~d}$ & Jan 14.5 & 200 & 28 & 12 & 8 & 6 & 0.019290 \\
\hline $2002 \mathrm{cr}$ & $-7 d$ & May 8.4 & 200 & 153 & 133 & 77 & 40 & 0.009590 \\
\hline $2002 \mathrm{cr}$ & $-5 \mathrm{~d}$ & May 10.3 & 200 & 53 & 37 & 11 & 4 & 0.009590 \\
\hline $2002 \mathrm{ef}$ & $+11 d$ & Aug 20.6 & 200 & 22 & 7 & 5 & 2 & 0.023977 \\
\hline $2002 \mathrm{el}$ & $-4 d$ & Aug 20.5 & 750 & 6 & 7 & 4 & 4 & 0.023300 \\
\hline $2002 \mathrm{fk}$ & $-14 d$ & Sep 19.4 & 1200 & 74 & 67 & 47 & 25 & 0.007125 \\
\hline $2002 \mathrm{fk}$ & $+42 d$ & Nov 14.4 & 750 & 37 & 13 & 15 & 10 & 0.007125 \\
\hline 2002ha & $+8 \mathrm{~d}$ & Nov 14.2 & 200 & 60 & 23 & 13 & 10 & 0.014046 \\
\hline $2002 \mathrm{hw}$ & $-1 d$ & Nov 14.3 & 1200 & 12 & 12 & 6 & 4 & 0.017535 \\
\hline $2003 W$ & $-8 \mathrm{~d}$ & Feb 2.4 & 200 & 104 & 29 & 47 & 22 & 0.020071 \\
\hline $2003 \mathrm{~W}$ & $-7 d$ & Feb 3.4 & 200 & 144 & 132 & 41 & 25 & 0.020071 \\
\hline $2003 \mathrm{cg}$ & $+37 d$ & May 9.2 & 200 & 66 & 56 & 80 & 47 & 0.004130 \\
\hline $2003 \mathrm{du}$ & $+1 \mathrm{~d}$ & May 9.3 & 1200 & 47 & 31 & 24 & 15 & 0.016764 \\
\hline $2003 d u$ & $+75 d$ & Jul 22.3 & 200 & 41 & 7 & 8 & 2 & 0.016764 \\
\hline 2004E & $+35 \mathrm{~d}$ & Feb 21.5 & 200 & 42 & 13 & 6 & 1 & 0.029807 \\
\hline $2004 \mathrm{ab}$ & $+18 d$ & Mar 7.5 & 750 & 41 & 27 & 45 & 36 & 0.005807 \\
\hline $2004 \mathrm{bk}$ & $+19 \mathrm{~d}$ & May 8.5 & 200 & 15 & 8 & 2 & 0 & 0.023036 \\
\hline $2004 \mathrm{bl}$ & $-2 \mathrm{~d}$ & May 8.3 & 1200 & 7 & 7 & 4 & 3 & 0.017319 \\
\hline $2004 \mathrm{bw}$ & $-9 \mathrm{~d}$ & May 30.4 & 200 & 74 & 54 & 17 & 12 & 0.021408 \\
\hline $2004 \mathrm{bv}$ & $-5 \mathrm{~d}$ & May 30.5 & 1200 & 91 & 69 & 54 & 37 & 0.010544 \\
\hline $2004 \mathrm{bv}$ & $+53 \mathrm{~d}$ & Jul 28.3 & 200 & 63 & 45 & 38 & 16 & 0.010544 \\
\hline $2004 \mathrm{ca}$ & $+46 \mathrm{~d}$ & Jul 28.6 & 200 & 56 & 24 & 13 & 5 & 0.017806 \\
\hline $2004 d a$ & $+4 d$ & Jul 8.5 & 750 & 8 & 8 & 6 & 5 & 0.016308 \\
\hline $2004 d a$ & $+14 d$ & Jul 18.4 & 750 & 4 & 2 & 5 & 5 & 0.016308 \\
\hline $2004 d a$ & $+18 d$ & Jul 22.5 & 750 & 35 & 15 & 30 & 23 & 0.016308 \\
\hline $2004 d a$ & $+24 d$ & Jul 28.4 & 750 & 18 & 10 & 15 & 10 & 0.016308 \\
\hline 2005am & $-4 d$ & Mar 5.3 & 1200 & 20 & 21 & 18 & 12 & 0.007889 \\
\hline $2005 \mathrm{am}$ & $+0 \mathrm{~d}$ & Mar 9.4 & 1200 & 30 & 32 & 22 & 16 & 0.007889 \\
\hline 2005am & $+7 \mathrm{~d}$ & Mar 16.4 & 750 & 3 & 2 & 2 & 3 & 0.007889 \\
\hline 2005am & $+14 d$ & Mar 23.3 & 1200 & 26 & 15 & 19 & 13 & 0.007889 \\
\hline
\end{tabular}

but care must be taken when comparing the relative strengths of features from widely separated wavelength regions.

Figure 11 shows the spectrum from SN 2002fk at $-14 d$, plotted in linear space and also in log space. Spectral features near 1.6 and $2.1 \mu \mathrm{m}$ are clearly smaller than the features appearing at 1.05 and $1.22 \mu \mathrm{m}$ when plotted in linear space, but these features appear to be larger when plotted in log space.

Acceleration of matter in the explosion lasts for only a few seconds but within minutes the envelope expands by several orders of magnitude from the initial WD radius (Khokhlov 1991; Höflich et al. 1993). The geometry of the expanding SN is assumed to be approximately spherical because the progenitor WD was nearly spherical, and the ejecta continue to move in homologous expansion with radial velocities proportional to the radial distances from the center (Khokhlov et al. 1997; Gamezo et al. 2003); see discussion in Section 5. A continuous range of expansion velocities can therefore be used to represent contiguous physical space in the $\mathrm{SN}$, and we often refer to comparative radial distances by their measured velocities. For example, a layer, or shell, of material in the spherical envelope may be described as occupying a region in space between 10,000 and $12,000 \mathrm{~km} \mathrm{~s}^{-1}$.

Polarization studies indicate that some SNe Ia, at least in the outer layers, may not be completely spherical (Wang et al. 2003; Wang \& Wheeler 2008). Possible explanations for the asphericity include rotation effects of the progenitor, mergers of two C/O WD stars, high-velocity filaments of explosion products, and influences on the ejecta by the binary companion. The observed polarization appears to be at maximum in high-velocity Ca II regions. The fact that high-velocity Ca II components are present in most of the early spectra in our sample suggests that NIR data will be valuable for further study of asymmetry in SNe Ia.

After a preliminary identification of an association between a spectral feature and an individual atomic line, it is prudent to examine the spectrum for other lines from the same ion. Comparison of relative line strengths can establish whether other lines from that ion would be expected to produce detectable features and confirm the initial identification. To easily make these 


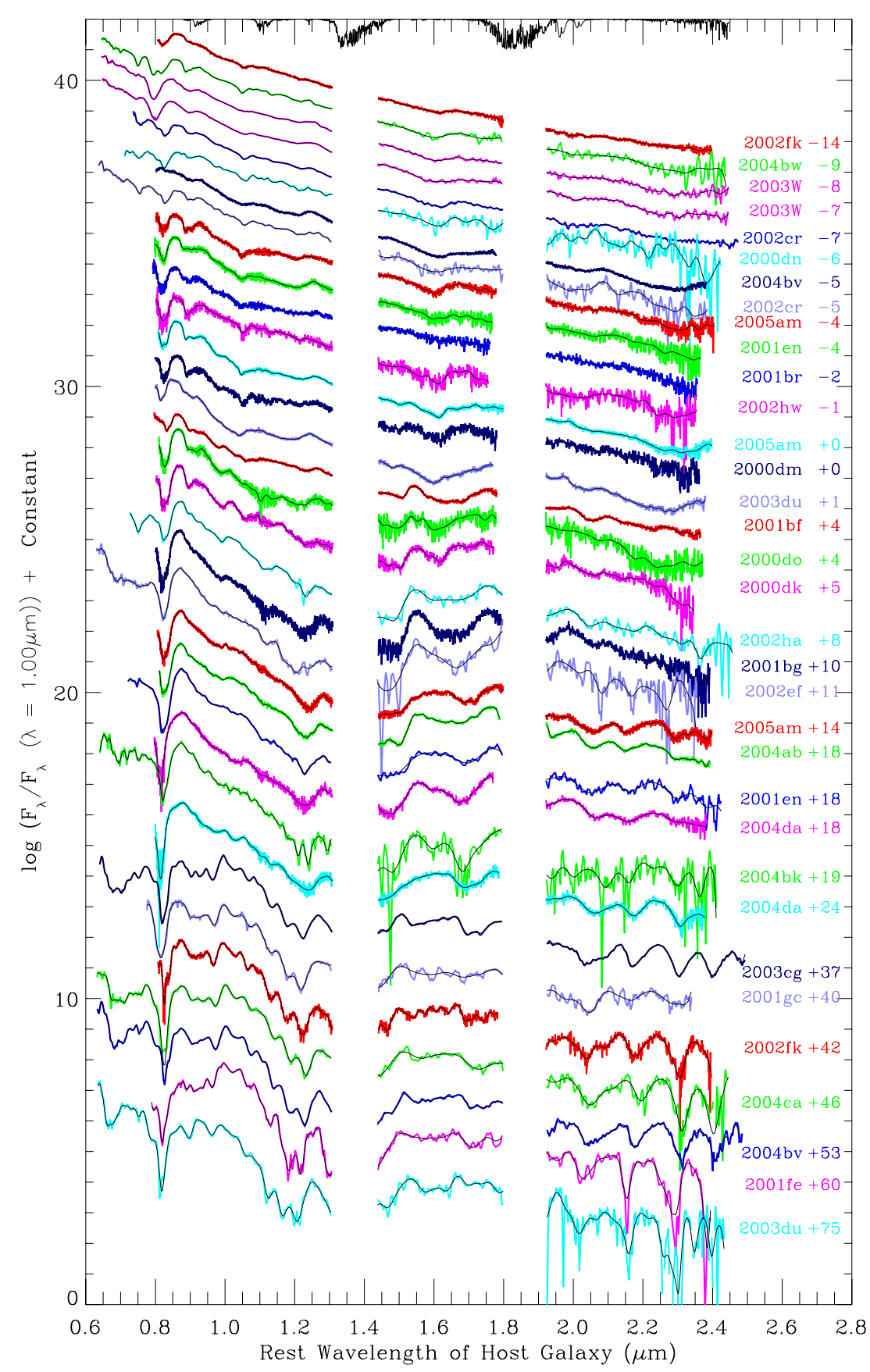

Figure 10. Thirty-four spectra selected from our sample for $\mathrm{S} / \mathrm{N}$ greater than 10 in the region $1.10-1.30 \mu \mathrm{m}$ (see Tables 2 and 3). These spectra cover the entire temporal range of our sample, from -14 to +75 days with respect to $V_{\max }$ (see the text in Section 3 ). The abscissa is wavelength, in microns, shifted to the rest frame of the host galaxy and the ordinate is log flux. All spectra have been normalized to 1.0 at $1.0 \mu \mathrm{m}$ and shifted by a constant for clarity.

(A color version of this figure is available in the online journal.)

comparisons, we have calculated line-strength estimates for several of the strongest lines at two temperatures: $5000 \mathrm{~K}$, which represents the temperatures found in extended line-forming regions, and also at $10,000 \mathrm{~K}$, which represents temperatures found closer to the photosphere. These temperatures are sufficiently separated to explore the effect of temperature on the relative strengths of lines from the same ion. It is not our intention to precisely define temperatures in the line-forming regions.

The relative line-strength estimate uses the local thermodynamic equilibrium (LTE) approximation for the fraction of ions from a population that will be excited to the $n$th level. The population of level $n$ is given by the Boltzmann factor:
$N_{n} \propto g_{n} f_{n} \times 10^{-\chi_{n} / k T}$, where $f_{n}$ is the oscillator strength, $g_{n}$ is the statistical weight, $\chi_{n}$ is the excitation potential, and $T$ is the temperature.

In Table 5, the estimated line strengths for ions is ordered by strength at $5000 \mathrm{~K}$ and in Table 6 , they are ordered by strength at $10,000 \mathrm{~K}$. Computed values for different lines from each ion are normalized to the strongest line and rest wavelengths are given in air. In situations where multiple lines from the same ion are close in wavelength, we calculate a mean value, weighted by oscillator strength, for two or more lines. For these blends, the $\log (g f)$ value and excitation minima are listed for the strongest line in the blend. Our generalized treatment of line-strength 


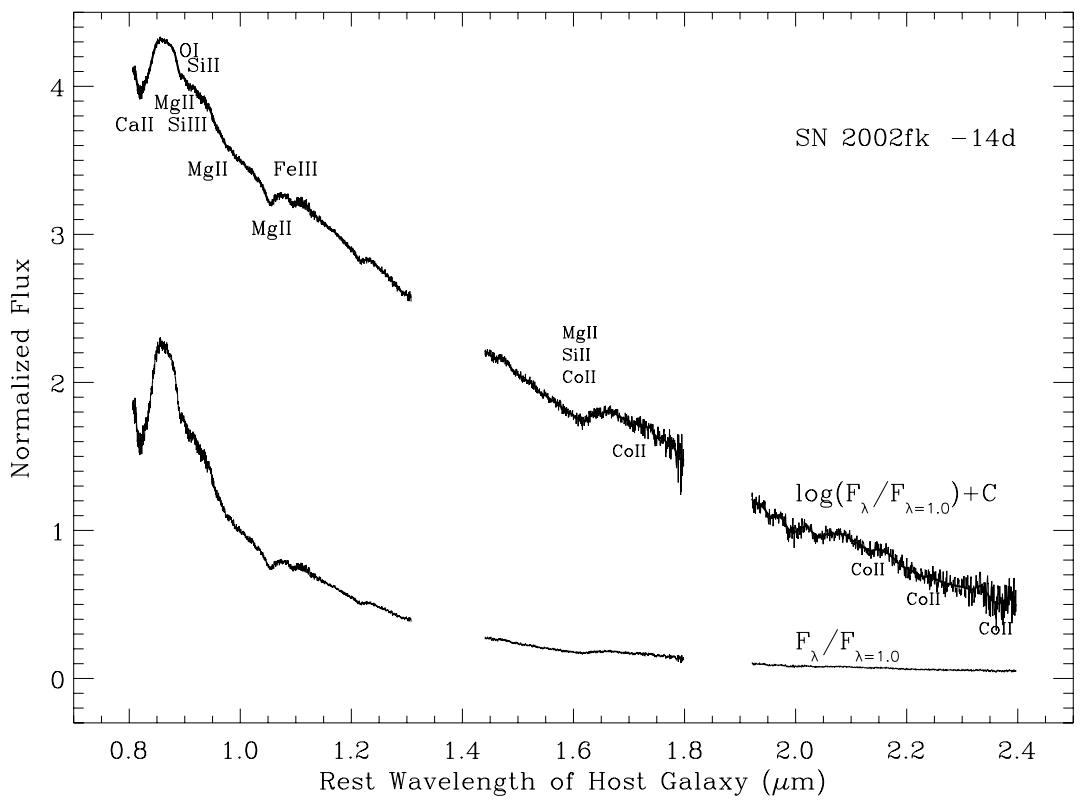

Figure 11. Very early spectrum of SN Ia 2002fk obtained 14 days before $V_{\max }$; only 5 or 6 days after the explosion. The data are displayed in both linear and log space (plus a constant) to demonstrate how the sizes of features at longer wavelengths are exaggerated by the log scale. Features are labeled for which we have reasonably confident identifications. A general discussion of this spectrum can be found in Section 3.1.1 and a more detailed analysis is found in Appendix A.1.

estimates has limitations but it is not model dependent. The use of non-LTE departure coefficients introduces uncertainty in the estimated line strengths by factors of 2-10. Uncertainties of this magnitude do not change our results.

Transitions from one ionization stage to another are expected to be abrupt in both time and velocity space due to the dominance of radiative transitions over collisional processes. Fe-group lines dominate the photoionization spectrum, so ionization boundaries for other elements are influenced by flux levels from the $\mathrm{Fe}$ transitions. Consequently, most line-forming regions will contain only one ionization stage for each atom (Marion et al. 2006).

\subsubsection{A Very Early Spectrum $(-14 d)$}

Figure 11 shows the spectrum of SN 2002fk, obtained 14 days before $V_{\max }$ which is only 5 or 6 days after the explosion. This is a single spectrum obtained in SXD mode. For a detailed discussion of individual features, please see Appendix A.1.

Probable identifications. $\mathrm{Mg}$ II, Si II, and Ca II.

Possible identifications. O I, Si III, Fe II, Fe III, and Co II.

Synopsis. The measured Doppler velocities in this spectrum are $10,000-12,000 \mathrm{~km} \mathrm{~s}^{-1}$, which are slower than the measured velocities for spectra from other SNe Ia obtained at later epochs. There is also possible evidence for multiple ionization stages of silicon. These unusual characteristics may be caused by lower temperatures within the envelope at this early epoch than will be found a few days later. The lower temperatures allow the photosphere to temporarily recede more deeply into the ejecta. Soon after the epoch of this spectrum, energy deposition from radioactive decay of ${ }^{56} \mathrm{Ni}$ increases the temperature and opacity which pushes the photosphere out to higher velocities (a more detailed discussion of this effect is found in Section 5.3).

$\mathrm{He}_{\mathrm{I}}$ and $\mathrm{C} \mathrm{I}$ are not detected in this spectrum. $\mathrm{O}_{\mathrm{I}}$ is possible from the $0.9264 \mu \mathrm{m}$ line at a Doppler velocity of $11,500 \mathrm{~km} \mathrm{~s}^{-1}$ in a blend with $\mathrm{Mg}$ II. $\mathrm{Mg}$ II is clearly detected from lines at 0.9227 and $1.0927 \mu \mathrm{m}$ and is probable from lines at 1.0092 and $1.6787 \mu \mathrm{m}$. Doppler velocities for $\mathrm{Mg}_{\mathrm{II}}$ are 10,300 $11,600 \mathrm{~km} \mathrm{~s}^{-1}$. A feature due to Si II from the $0.9413 \mu \mathrm{m}$ line is likely to be present at $11,300 \mathrm{~km} \mathrm{~s}^{-1}$. Si III is possible but at a low Doppler velocity of $8300 \mathrm{~km} \mathrm{~s}^{-1}$. The Ca II IR triplet is strong at $12,300 \mathrm{~km} \mathrm{~s}^{-1}$. Fe II is unlikely and $\mathrm{Fe}$ III is possible but at a low Doppler velocity of $8100 \mathrm{~km} \mathrm{~s}^{-1}$. Co II is detected at $12,000 \mathrm{~km} \mathrm{~s}^{-1}$.

\subsubsection{The Photospheric Phase (Outer Layers; $-14 d$ to $+5 d$ )}

Figure 12 shows 18 spectra obtained between -14 and +5 days relative to $V_{\max }$. During this epoch, the photosphere is expected to be in the outer layers of the $\mathrm{SN}$ and the line-forming regions will be close to the photosphere. $\mathrm{Mg}$ II is prominent from multiple lines in all spectra in this group. Six of the spectra were obtained in LRS mode which extends blueward to about $0.7 \mu \mathrm{m}$ at $R=200$ (compared with $R=1200$ for the SXD mode). Figure 13 shows four representative spectra from this group: SNe 2004bw (LRS obtained at -9d), 2005am (SXD obtained at $-4 \mathrm{~d}$ ), 2000dm (SXD obtained at $+0 \mathrm{~d}$ ), and 2001bf (SXD obtained at $+4 \mathrm{~d}$ ). Only the region $0.70-1.32 \mu \mathrm{m}$ is displayed in Figures 12 and 13 in order to enhance details in the area where most of the features are under discussion. Features are labeled in the figure for which we have reasonably confident identifications. For a detailed discussion of individual features from Photospheric Phase group, please see Appendix A.2.

Velocity references are compiled in Table 4. Probable identifications. O I, Mg II, Si II, and Ca II. Possible identifications. Mn II, Fe II, Fe III, and Co II. Synopsis. Doppler velocities for lines identified in this group are between 10,000 and $14,000 \mathrm{~km} \mathrm{~s}^{-1}$ with lower velocities from both spectra of SN 2002cr ( $-7 \mathrm{~d}$ and $-5 \mathrm{~d})$ and from SN $2001 \mathrm{bf}$ $(+4 d)$.

$\mathrm{C}_{\mathrm{I}}$ is not detected in any of the spectra (Section 4.3). Strong features near $0.75 \mu \mathrm{m}$ due to $\mathrm{O}$ I are detected in all spectra in this group that include the wavelength region. $\mathrm{Mg}$ II produces multiple features in all spectra from this group and the $\mathrm{Mg}$ II lines clearly correlate by velocity and strength. The Ca II IR triplet is prominent in all spectra from this group and a highvelocity component of the Ca II feature is present to some extent in all spectra that include the wavelength region shorter than $0.8 \mu \mathrm{m}$. Si II is detected either alone or in a blend with Mn II. Fe II is likely in blends with other atoms, as is Co II. 
Table 4

Measured Doppler Velocities for Identified Lines-Blueshift $\left(\mathrm{km} \mathrm{s}^{-1}\right)$

\begin{tabular}{|c|c|c|c|c|c|c|c|c|c|c|}
\hline Name & $\begin{array}{c}\text { Epoch } \\
V_{\max }\end{array}$ & $\begin{array}{c}\text { O I } \\
0.7773 \\
\end{array}$ & $\begin{array}{c}\text { O I } \\
0.9264 \\
\end{array}$ & $\begin{array}{c}\mathrm{Mg}_{\text {II }} \\
0.9227 \\
\end{array}$ & $\begin{array}{c}\mathrm{Mg}_{\mathrm{II}} \\
1.0092 \\
\end{array}$ & $\begin{array}{c}\mathrm{Mg}_{\text {II }} \\
1.0927 \\
\end{array}$ & $\begin{array}{c}\text { Si II } \\
0.9413 \\
\end{array}$ & $\begin{array}{c}S_{\text {I }} \\
0.9223 \\
\end{array}$ & $\begin{array}{c}\text { Ca II } \\
0.8538 \\
\end{array}$ & $\begin{array}{c}\text { Fe II } \\
0.9998 \\
\end{array}$ \\
\hline $2002 \mathrm{fk}$ & -14 & & 11,100 & 9,900 & 10,800 & 11,300 & 10,800 & & 12,600 & \\
\hline $2004 \mathrm{bw}$ & -9 & 11,300 & 13,000 & 11,800 & 13,400 & 12,500 & & & 13,000 & 10,600 \\
\hline $2003 W$ & -8 & 13,500 & 11,000 & 9,800 & 12,600 & 14,500 & & & & 9,700 \\
\hline $2003 W$ & -7 & 13,300 & 11,000 & 9,800 & 14,800 & 11,000 & & & & 12,000 \\
\hline $2002 \mathrm{cr}$ & -7 & 9,300 & 11,000 & 9,800 & 12,600 & 11,100 & 11,600 & & 9,800 & 9,700 \\
\hline $2000 \mathrm{dn}$ & -6 & 11,800 & 11,300 & 10,100 & 12,800 & 12,500 & 10,400 & & 10,400 & 10,000 \\
\hline 2004 bv & -5 & & 11,600 & 10,400 & 14,400 & 12,400 & 11,500 & 10,200 & 8,900 & \\
\hline $2002 \mathrm{cr}$ & -5 & 9,400 & 10,700 & 9,500 & 12,200 & 11,200 & & & 9,600 & 9,400 \\
\hline $2005 \mathrm{am}$ & -4 & & 13,200 & 12,000 & 13,400 & 12,900 & 12,100 & & 12,100 & 10,600 \\
\hline 2001en & -4 & & 14,800 & 13,600 & 13,300 & 13,500 & 12,800 & & 11,400 & 10,500 \\
\hline $2001 \mathrm{br}$ & -2 & & 15,700 & 14,500 & 11,900 & 13,700 & 14,100 & & 14,200 & 9,100 \\
\hline $2002 \mathrm{hw}$ & -1 & & 11,900 & 10,700 & 12,900 & 11,900 & 10,200 & & 12,200 & \\
\hline $2005 \mathrm{am}$ & +0 & & 13,100 & 11,900 & 11,000 & 13,100 & 10,400 & 11,700 & 12,500 & 7,700 \\
\hline $2000 \mathrm{dm}$ & +0 & & 11,600 & 10,400 & 9,500 & 11,500 & & 10,300 & 10,900 & 6,700 \\
\hline $2003 \mathrm{du}$ & +1 & & 15,000 & 13,800 & 13,500 & 14,900 & 10,800 & 13,700 & 14,200 & \\
\hline $2001 b f$ & +4 & & 9,800 & 8,600 & 9,700 & 9,600 & 7,400 & 8,500 & 7,600 & 6,800 \\
\hline 2000 do & +4 & & 11,700 & 10,700 & & 11,600 & 7,300 & 9,400 & 10,400 & 3,700 \\
\hline $2000 \mathrm{dk}$ & +5 & & 10,800 & 9,600 & 9,000 & 10,800 & & 9,500 & 11,600 & 2,500 \\
\hline 2002 ha & +8 & 10,200 & 11,300 & & & & & 10,000 & 11,300 & 3,100 \\
\hline $2001 \mathrm{bg}$ & +10 & & 12,300 & & & & 10,500 & 11,000 & 12,300 & 3,100 \\
\hline $2002 \mathrm{ef}$ & +11 & 9,800 & & & & & & & 11,400 & 2,300 \\
\hline $2005 \mathrm{am}$ & +14 & & 10,500 & & & & & 9,200 & 11,400 & 2,900 \\
\hline $2004 a b$ & +18 & & 10,300 & & & & 8,200 & 8,900 & 12,200 & 2,600 \\
\hline 2002en & +18 & 12,300 & & & & & 7,500 & & 13,000 & \\
\hline
\end{tabular}

Note. These data are plotted in Figure 19.

A bump appearing near $1.25 \mu \mathrm{m}$ is strongly peaked in some of the spectra, unlike emission from a P Cygni profile. This feature does not have a confident identification although it is observed in all spectra throughout our entire sample from $-14 \mathrm{~d}$ to $+75 \mathrm{~d}$. The feature near $0.97 \mu \mathrm{m}$ becomes stronger after maximum light due to contributions from Fe II. Another feature of uncertain origin appears at the same time near $1.48 \mu \mathrm{m}$. Beginning about maximum brightness, the spectra begin to be affected by changes in the apparent continuum due to line blanketing from Fe-group lines. "Emission" bumps begin to appear near 1.25, 1.52, and from 1.6 to $2.1 \mu \mathrm{m}$ and in broader regions (see discussion in Section 5.3).

\subsubsection{The Extended Photospheric Phase (Intermediate Layers; $+4 d$ to $+24 d)$}

Figure 14 shows 10 spectra from SNe Ia obtained between 4 and 24 days after $V_{\max }$. During the phase represented by this group, the shape of the continuum becomes influenced by lineblanketing from $\mathrm{Fe}$-group lines which extends the effective photospheric radius (see Section 5.3). Spectra obtained in LRS mode extend blueward of $0.80 \mu \mathrm{m}$ and all others were obtained in SXD mode. Figure 15 shows spectra from SNe 2000dk (+5d), 2001bg (+10d), SN 2004ab (+18d), and SN 2004da $(+24 d)$ as representative of this group with features labeled for which we have confident identifications. For a detailed discussion of individual features from this group, please reference Appendix A.3.

Velocity references are compiled in Table 4. Probable identifications. O I, Mg II, Si II, S I, Ca II, Fe II, and Co II.

Possible identifications. Si I and Mn II.

Synopsis. During the temporal period represented by this group, transitions are made from spectra dominated by $\mathrm{O}_{\mathrm{I}}$ and $\mathrm{Mg}$ II lines to spectra with lines from S I, Fe II, and possibly Mn II and
Si I. Measured Doppler velocities remain above $9000 \mathrm{~km} \mathrm{~s}^{-1}$ for most ions with the exception of Fe II which begins near $9000 \mathrm{~km} \mathrm{~s}^{-1}$ at earlier epochs but slows to less than $3000 \mathrm{~km} \mathrm{~s}^{-1}$ by $+18 \mathrm{~d}$.

O I continues to be detected up to $+10 \mathrm{~d}$ and is possibly identified to $+18 \mathrm{~d}$. $\mathrm{Mg}$ II is likely up to $+5 \mathrm{~d}$ but is absent thereafter. The absorption near $0.89 \mu \mathrm{m}$ shifts to 0.90 and apparently makes a transition from $\mathrm{Mg}$ II to $\mathrm{S}_{\mathrm{I}}$ with possible contributions from $\mathrm{O}$ I. This feature becomes much weaker after $+5 \mathrm{~d}$ which is consistent with losing the signal from $\mathrm{Mg}$ II and $\mathrm{O}$ I.

Si II and Mn II are possible alone or in a blend producing the feature found near $0.92 \mu \mathrm{m}$ that is not present in all spectra. The Ca II IR triplet remains prominent and the data continue to separately resolve the two strongest components of this feature. Fe II is detected from the line at $0.9998 \mu \mathrm{m}$ in all spectra through $+18 \mathrm{~d}$, and Co II is strong in the spectra from this group obtained at $+14 d$ and later.

Individual $\mathrm{Fe}$-group lines are complicated by many potential transitions at the same wavelength and nearly the same strength within each multiplet so the simple formula we have been using to estimate line strength may not be accurate for Fe II as it was for smaller atoms. In the spectra from this group, the $0.9998 \mu \mathrm{m}$ line is the only $\mathrm{Fe}$ II line detected $(+4 \mathrm{~d}$ through $+18 \mathrm{~d})$, although the calculated line strengths suggest that lines at 0.7462, 0.7712, and $1.0500 \mu \mathrm{m}$ should also be detected (Appendix A.3).

During the Extended Photospheric Phase, the shape of the continuum becomes influenced by line-blanketing from $\mathrm{Fe}$ group lines (see Section 5.3). Looking at this group in the context of the entire sample (Figure 10), we can see how unresolved groups of Fe lines change the effective shape of the continuum during the epoch covered by these spectra. Significant peaks arise near $1.25,1.55,1.75,2.0,2.1$, and $2.25 \mu \mathrm{m}$. Thus, many of the apparent "absorption" features may be interpreted as gaps between regions of inflating continuum. For example, minima 
Table 5

Estimated Line Strengths at 5000 K in the Region 0.7-2.4 $\mu \mathrm{m}$

\begin{tabular}{|c|c|c|c|c|c|c|}
\hline Ion & $\begin{array}{c}\lambda_{\text {rest }}(\text { air }) \\
(\mu \mathrm{m})\end{array}$ & $\begin{array}{l}\text { No. of Lines } \\
\text { in Blend }\end{array}$ & $\log (g f)$ & $\begin{array}{l}\text { Excitation } \\
\text { Min. }(\mathrm{eV})\end{array}$ & $\begin{array}{c}\text { Line Strength } \\
5000 \mathrm{~K}^{\mathrm{b}}\end{array}$ & $\begin{array}{c}\text { Line Strength } \\
10,000 \mathrm{~K}^{\mathrm{b}}\end{array}$ \\
\hline$\overline{\mathrm{He}} \mathrm{I}$ & 1.0830 & 3 & -0.047 & 19.82096 & 15.1 & 6.0 \\
\hline $\mathrm{He} \mathrm{I}$ & 2.0581 & 1 & -0.424 & 20.61718 & 1.000 & 1.000 \\
\hline$\underline{\mathrm{He}} \mathrm{I}$ & 0.7065 & 3 & -0.460 & 20.96551 & 0.410 & 0.614 \\
\hline $\mathrm{C}_{\mathrm{I}}$ & 1.0693 & 7 & 0.348 & 7.48830 & 1.000 & 1.000 \\
\hline $\mathrm{C}_{\mathrm{I}}$ & 0.9093 & 2 & 0.142 & 7.48830 & 0.622 & 0.622 \\
\hline $\mathrm{C}_{\mathrm{I}}$ & 0.9406 & 1 & 0.225 & 7.68529 & 0.477 & 0.599 \\
\hline $\mathrm{C}_{\mathrm{I}}$ & 0.9658 & 1 & -0.264 & 7.48830 & 0.244 & 0.244 \\
\hline $\mathrm{C}_{\mathrm{I}}$ & 1.4543 & 1 & -0.110 & 7.68529 & 0.221 & 0.277 \\
\hline $\mathrm{C}_{\mathrm{I}}$ & 1.1754 & 2 & 0.661 & 8.64774 & 0.139 & 0.535 \\
\hline $\mathrm{C}_{\mathrm{I}}$ & 0.8335 & 1 & -0.420 & 7.68529 & 0.108 & 0.136 \\
\hline $\mathrm{C}_{\mathrm{I}}$ & 1.1330 & 1 & 0.280 & 8.53767 & 0.075 & 0.253 \\
\hline $\mathrm{C}_{\text {II }}$ & 0.6580 & 2 & 0.118 & 14.44980 & 88,393 & 253 \\
\hline $\mathrm{C}_{\text {II }}$ & 0.7235 & 3 & 0.330 & 16.33422 & 1,815 & 46.3 \\
\hline $\mathrm{C}_{\text {II }}$ & 1.8905 & 2 & 0.258 & 19.49586 & 1.000 & 0.959 \\
\hline $\mathrm{C}_{\text {II }}$ & 1.7846 & 3 & 0.550 & 20.15184 & 0.427 & 0.878 \\
\hline $\mathrm{C}_{\text {II }}$ & 0.9903 & 3 & 1.010 & 20.95206 & 0.192 & 1.000 \\
\hline $\mathrm{C}_{\mathrm{II}}$ & 0.9230 & 3 & 0.620 & 20.84618 & 0.100 & 0.461 \\
\hline O I & 0.7773 & 3 & 0.324 & 9.14671 & 1.000 & 1.000 \\
\hline $\mathrm{OI}_{\mathrm{I}}$ & 0.8446 & 3 & 0.170 & 9.52201 & 0.294 & 0.454 \\
\hline $\mathrm{OI}_{\mathrm{I}}$ & 0.9264 & 9 & 0.690 & 10.74166 & 0.057 & 0.365 \\
\hline O I & 1.1290 & 9 & 0.500 & 10.98960 & 0.021 & 0.177 \\
\hline $\mathrm{O}_{\mathrm{I}}$ & 0.7990 & 6 & 0.280 & 10.98960 & 0.013 & 0.106 \\
\hline $\mathrm{O}_{\mathrm{I}}$ & 1.3165 & 3 & -0.095 & 10.98960 & 0.005 & 0.045 \\
\hline O II & 0.6695 & 2 & -0.594 & 23.44307 & 2,155 & 97.6 \\
\hline $\mathrm{O}_{\text {II }}$ & 2.1085 & 2 & -1.690 & 25.66290 & 1.000 & 0.595 \\
\hline $\mathrm{O}_{\mathrm{II}}$ & 1.3811 & 3 & -1.730 & 25.66290 & 0.912 & 0.543 \\
\hline $\mathrm{O}_{\text {II }}$ & 1.4008 & 2 & 0.530 & 29.62095 & 0.017 & 1.000 \\
\hline O II & 0.7114 & 3 & -0.170 & 29.07061 & 0.012 & 0.378 \\
\hline $\mathrm{O}_{\mathrm{II}}$ & 1.3029 & 2 & 0.460 & 29.82223 & 0.009 & 0.674 \\
\hline O II & 1.2500 & 1 & 0.210 & 29.82223 & 0.005 & 0.379 \\
\hline$\underline{\mathrm{O}} \mathrm{II}$ & 1.1667 & 2 & 0.870 & 30.50576 & 0.005 & 0.784 \\
\hline $\mathrm{Mg}_{\mathrm{I}}$ & 0.8807 & 1 & -0.137 & 4.34610 & 1.000 & 0.807 \\
\hline $\mathrm{Mg}_{\mathrm{I}}$ & 1.1828 & 1 & -0.290 & 4.34610 & 0.703 & 0.568 \\
\hline $\mathrm{Mg}_{\mathrm{I}}$ & 1.5033 & 3 & 0.340 & 5.10817 & 0.511 & 1.000 \\
\hline $\mathrm{Mg}_{\mathrm{I}}$ & 1.7109 & 1 & 0.140 & 5.39409 & 0.166 & 0.453 \\
\hline $\mathrm{Mg}_{\mathrm{I}}$ & 1.4878 & 9 & 0.660 & 5.94632 & 0.153 & 0.790 \\
\hline$\underline{\mathrm{Mg}_{\mathrm{I}}}$ & 1.2083 & 4 & 0.450 & 5.75363 & 0.147 & 0.609 \\
\hline Mg II & 0.9227 & 2 & 0.270 & 8.65529 & 1.000 & 1.000 \\
\hline Mg II & 1.0927 & 3 & 0.020 & 8.86425 & 0.346 & 0.441 \\
\hline $\mathrm{Mg}_{\text {II }}$ & 0.7890 & 3 & 0.650 & 10.00000 & 0.106 & 0.504 \\
\hline Mg II & 0.8228 & 2 & 0.030 & 10.00000 & 0.025 & 0.121 \\
\hline Mg II & 1.0092 & 3 & 1.020 & 11.63047 & 0.006 & 0.178 \\
\hline $\mathrm{Mg}_{\text {II }}$ & 0.9632 & 3 & 0.660 & 11.56982 & 0.003 & 0.083 \\
\hline $\mathrm{Mg}_{\text {II }}$ & 2.1369 & 1 & 0.390 & 11.50533 & 0.002 & 0.048 \\
\hline$\underline{\mathrm{Mg} \text { II }}$ & 1.6787 & 3 & 0.730 & 12.08542 & 0.001 & 0.054 \\
\hline Si I & 1.2032 & 1 & 0.440 & 4.95413 & 1.000 & 1.000 \\
\hline Si I & 1.0827 & 1 & 0.220 & 4.95413 & 0.603 & 0.603 \\
\hline Si I & 1.0585 & 1 & -0.020 & 4.95413 & 0.347 & 0.347 \\
\hline$\underline{\mathrm{Si} I}$ & 1.5888 & 1 & -0.030 & 5.08269 & 0.251 & 0.292 \\
\hline Si II & 0.6355 & 2 & 0.297 & 8.12157 & 10,126 & 49.6 \\
\hline Si II & 1.6930 & 2 & 0.350 & 12.14781 & 1.000 & 0.524 \\
\hline Si II & 0.9413 & 3 & 0.980 & 12.84018 & 0.855 & 1.000 \\
\hline Si II & 0.7849 & 3 & 0.490 & 12.52627 & 0.573 & 0.466 \\
\hline Si II & 1.1737 & 3 & 0.620 & 12.88100 & 0.340 & 0.416 \\
\hline Si II & 1.3681 & 2 & 0.130 & 12.88100 & 0.110 & 0.135 \\
\hline Si II & 1.7183 & 3 & 0.890 & 14.10541 & 0.037 & 0.187 \\
\hline$\underline{\text { Si II }}$ & 1.1311 & 3 & -0.460 & 12.84018 & 0.031 & 0.036 \\
\hline Si III & 0.9324 & 1 & -0.120 & 20.55363 & 7,223 & 41.6 \\
\hline Si III & 1.0526 & 1 & -2.950 & 20.55363 & 10.7 & 0.062 \\
\hline Si III & 1.2523 & 3 & 0.500 & 24.99684 & 1.000 & 1.000 \\
\hline Si III & 0.7465 & 6 & 0.360 & 24.99684 & 0.724 & 0.724 \\
\hline
\end{tabular}


Table 5

(Continued)

\begin{tabular}{|c|c|c|c|c|c|c|}
\hline Ion & $\begin{array}{c}\lambda_{\text {rest }} \text { (air) } \\
(\mu \mathrm{m})\end{array}$ & $\begin{array}{l}\text { No. of Lines } \\
\text { in Blend }\end{array}$ & $\log (g f)$ & $\begin{array}{l}\text { Excitation } \\
\text { Min. }(\mathrm{eV})\end{array}$ & $\begin{array}{l}\text { Line Strength } \\
5000 \mathrm{~K}^{\mathrm{b}}\end{array}$ & $\begin{array}{c}\text { Line Strength } \\
10,000 \mathrm{~K}^{\mathrm{b}} \\
\end{array}$ \\
\hline Si III & 1.2601 & 3 & 0.160 & 24.99662 & 0.457 & 0.457 \\
\hline Si III & 0.9800 & 1 & 0.230 & 25.33550 & 0.245 & 0.363 \\
\hline Si III & 1.1341 & 3 & 0.430 & 25.56430 & 0.228 & 0.441 \\
\hline$\overline{S_{I}}$ & 0.9223 & 3 & 0.420 & 6.52494 & 1.000 & 1.000 \\
\hline S I $_{1}$ & 1.0457 & 3 & 0.260 & 6.86061 & 0.317 & 0.469 \\
\hline $\mathrm{S}_{\mathrm{I}}$ & 1.8940 & 6 & 0.450 & 8.04620 & 0.031 & 0.183 \\
\hline$S_{\text {I }}$ & 2.2694 & 3 & 0.240 & 7.87043 & 0.029 & 0.139 \\
\hline$S_{\text {I }}$ & 1.3809 & 3 & 0.110 & 7.87043 & 0.022 & 0.103 \\
\hline $\mathrm{S}_{\text {II }}$ & 0.8315 & 1 & -0.470 & 14.06839 & 1.000 & 1.000 \\
\hline S II & 0.7967 & 1 & -0.820 & 14.00342 & 0.519 & 0.482 \\
\hline S II $_{\text {II }}$ & 0.7590 & 1 & -0.880 & 14.23489 & 0.264 & 0.321 \\
\hline $\mathrm{S}_{\text {II }}$ & 0.8855 & 2 & -1.110 & 14.16002 & 0.185 & 0.206 \\
\hline $\mathrm{S}_{\text {II }}$ & 1.4501 & 2 & -0.216 & 16.59204 & 0.005 & 0.096 \\
\hline S II & 1.3529 & 1 & -0.425 & 16.53575 & 0.004 & 0.063 \\
\hline $\mathrm{CaI}$ & 0.6149 & 3 & 0.100 & 1.89906 & 5.1 & 2.0 \\
\hline $\mathrm{Ca} I$ & 0.7148 & 1 & 0.208 & 2.70919 & 1.000 & 1.000 \\
\hline $\mathrm{Ca} I$ & 1.9753 & 4 & -0.831 & 1.89906 & 0.599 & 0.234 \\
\hline $\mathrm{Ca} I$ & 0.7326 & 1 & 0.073 & 2.93271 & 0.436 & 0.565 \\
\hline $\mathrm{Ca} I$ & 1.9453 & 1 & -1.094 & 1.88593 & 0.337 & 0.130 \\
\hline$\overline{\mathrm{Ca}}$ II & 0.8538 & 2 & -0.362 & 1.70005 & 13,944 & 40.3 \\
\hline $\mathrm{Ca}$ II & 0.8662 & 1 & -0.623 & 1.69252 & 7,780 & 22.3 \\
\hline $\mathrm{Ca}$ II & 1.1839 & 1 & 0.300 & 6.46831 & 1.000 & 0.731 \\
\hline Ca II & 0.8921 & 3 & 0.729 & 7.05003 & 0.696 & 1.000 \\
\hline Ca II & 1.1950 & 1 & 0.000 & 6.46831 & 0.501 & 0.367 \\
\hline Ca II & 0.8249 & 2 & 0.621 & 7.51535 & 0.184 & 0.454 \\
\hline $\mathrm{Ca}$ II & 0.8202 & 1 & 0.315 & 7.50564 & 0.093 & 0.227 \\
\hline Ca II & 0.9931 & 1 & 0.072 & 7.51535 & 0.052 & 0.128 \\
\hline$\overline{M n I}$ & 1.2900 & 1 & -1.059 & 2.11436 & 1.000 & 0.541 \\
\hline Mn I & 1.3310 & 2 & -1.360 & 2.14284 & 0.468 & 0.262 \\
\hline Mn I & 1.3630 & 3 & -1.516 & 2.16386 & 0.311 & 0.178 \\
\hline Mn I & 1.3859 & 2 & -1.638 & 2.17836 & 0.227 & 0.133 \\
\hline Mn I & 1.5184 & 2 & 0.606 & 4.88919 & 0.074 & 1.000 \\
\hline Mn I & 0.8740 & 3 & -0.055 & 4.43521 & 0.046 & 0.370 \\
\hline Mn I & 1.5263 & 1 & 0.379 & 4.88919 & 0.044 & 0.593 \\
\hline Mn I & 0.7309 & 3 & -0.084 & 4.43521 & 0.043 & 0.346 \\
\hline Mn II & 0.7414 & 3 & -2.202 & 3.70608 & 1.000 & 1.000 \\
\hline Mn II & 0.7342 & 2 & -2.713 & 3.70979 & 0.306 & 0.307 \\
\hline Mn II & 0.9447 & 1 & -2.389 & 4.06545 & 0.282 & 0.428 \\
\hline Mn II & 0.9388 & 2 & -2.554 & 4.07405 & 0.189 & 0.290 \\
\hline Mn II & 0.8695 & 1 & 0.577 & 9.24489 & 0.002 & 0.971 \\
\hline Mn II & 0.8769 & 1 & 0.491 & 9.24489 & 0.001 & 0.797 \\
\hline Mn II & 0.8820 & 1 & 0.347 & 9.24489 & 0.001 & 0.572 \\
\hline Mn II & 0.9486 & 2 & 0.491 & 9.46988 & 0.001 & 0.614 \\
\hline$\overline{\mathrm{Fe}} \mathrm{I}$ & 0.8677 & 2 & -1.212 & 2.17609 & 1.000 & 0.417 \\
\hline $\mathrm{Fe} I$ & 0.8824 & 1 & -1.364 & 2.19801 & 0.670 & 0.286 \\
\hline $\mathrm{Fe} I$ & 1.1973 & 1 & -1.476 & 2.17609 & 0.545 & 0.227 \\
\hline $\mathrm{Fe} I$ & 0.8388 & 1 & -1.493 & 2.17609 & 0.524 & 0.218 \\
\hline $\mathrm{Fe} I$ & 0.7511 & 5 & 0.107 & 4.17798 & 0.200 & 0.851 \\
\hline $\mathrm{Fe}_{\mathrm{I}}$ & 0.8220 & 2 & 0.249 & 4.32039 & 0.199 & 1.000 \\
\hline $\mathrm{Fe} I$ & 0.7196 & 3 & -0.120 & 4.10365 & 0.141 & 0.550 \\
\hline $\mathrm{Fe} I$ & 0.7941 & 2 & 0.154 & 4.38676 & 0.137 & 0.744 \\
\hline $\mathrm{Fe} I$ & 0.7495 & 2 & -0.102 & 4.22065 & 0.112 & 0.500 \\
\hline $\mathrm{Fe} I$ & 0.7999 & 1 & 0.048 & 4.37164 & 0.111 & 0.593 \\
\hline$\overline{\mathrm{Fe}}$ II & 0.7712 & 1 & -2.543 & 3.90368 & 1.000 & 1.000 \\
\hline Fe II & 0.7462 & 1 & -2.734 & 3.89187 & 0.662 & 0.653 \\
\hline Fe II & 0.9998 & 1 & -1.826 & 5.48450 & 0.133 & 0.832 \\
\hline Fe II & 1.0500 & 2 & -1.997 & 5.54914 & 0.077 & 0.521 \\
\hline Fe II & 1.0863 & 1 & -2.121 & 5.58957 & 0.053 & 0.374 \\
\hline $\mathrm{Fe}$ II & 1.1126 & 1 & -2.236 & 5.61560 & 0.038 & 0.278 \\
\hline Fe II & 0.7515 & 1 & -2.362 & 5.82361 & 0.018 & 0.163 \\
\hline $\mathrm{Fe}$ II & 0.9550 & 1 & -2.027 & 6.21915 & 0.015 & 0.223 \\
\hline
\end{tabular}


Table 5

(Continued)

\begin{tabular}{lcccccc}
\hline \hline Ion & $\begin{array}{c}\lambda_{\text {rest }}(\text { air }) \\
(\mu \mathrm{m})\end{array}$ & $\begin{array}{c}\text { No. of Lines } \\
\text { in Blend }^{\mathrm{a}}\end{array}$ & $\log (g f)$ & $\begin{array}{c}\text { Excitation } \\
\text { Min. }(\mathrm{eV})\end{array}$ & $\begin{array}{c}\text { Line Strength } \\
5000 \mathrm{~K}^{\mathrm{b}}\end{array}$ & $\begin{array}{c}\text { Line Strength } \\
10,000 \mathrm{~K}^{\mathrm{b}}\end{array}$ \\
\hline Fe III & 0.7261 & 1 & -2.910 & 13.13443 & 1.000 & 0.354 \\
Fe III & 0.7265 & 1 & -1.708 & 14.62496 & 0.501 & 1.000 \\
Fe III & 0.9124 & 1 & -2.577 & 14.62496 & 0.068 & 0.135 \\
Fe III & 1.6722 & 1 & 0.235 & 22.87010 & 0.000 & 0.006 \\
Fe III & 0.7456 & 1 & -1.905 & 20.88295 & 0.000 & 0.000 \\
Fe III & 1.6672 & 1 & 0.057 & 22.86637 & 0.000 & 0.004 \\
Fe III & 1.2039 & 2 & 0.663 & 23.61137 & 0.000 & 0.007 \\
Fe III & 1.2786 & 1 & 0.723 & 23.67243 & 0.000 & 0.007 \\
\hline Co I & 0.7085 & 1 & -1.018 & 1.88271 & 1.000 & 0.589 \\
Co I & 0.7053 & 1 & -1.440 & 1.95586 & 0.319 & 0.205 \\
Co I & 0.8098 & 4 & 0.290 & 4.02115 & 0.142 & 1.000 \\
Co I & 0.8027 & 6 & 0.116 & 4.14628 & 0.071 & 0.579 \\
Co I & 0.8374 & 2 & -0.040 & 4.07216 & 0.059 & 0.441 \\
\hline Co II & 1.7772 & 1 & -2.087 & 5.04595 & 1.000 & 0.736 \\
Co II & 1.7462 & 1 & -2.284 & 5.12265 & 0.532 & 0.428 \\
Co II & 2.2205 & 1 & -2.374 & 5.04595 & 0.516 & 0.380 \\
Co II & 2.4596 & 1 & -2.416 & 5.12265 & 0.392 & 0.316 \\
Co II & 2.3613 & 1 & -2.491 & 5.17524 & 0.292 & 0.250 \\
Co II & 1.6064 & 1 & -2.568 & 5.12265 & 0.276 & 0.223 \\
Co II & 1.5759 & 1 & -2.725 & 5.04595 & 0.230 & 0.169 \\
Co II & 1.6361 & 1 & -2.625 & 5.17524 & 0.215 & 0.184 \\
Co II & 1.7239 & 1 & -2.649 & 5.17524 & 0.203 & 0.174 \\
Co II & 2.2497 & 1 & -2.697 & 5.20879 & 0.168 & 0.150 \\
Co II & 2.1347 & 1 & -2.906 & 5.04595 & 0.152 & 0.003 \\
Co II & 0.8342 & 2 & 0.753 & 10.41714 & 0.002 & 0.112 \\
Co II & 0.8737 & 1 & 0.663 & 10.41714 & & 0.813 \\
\hline
\end{tabular}

Notes.

${ }^{\text {a }}$ If more than one line is included in the blend, the indicated wavelength is a mean value, weighted by oscillator strength. Some lines have been omitted from the table if their rest wavelengths are very close to that of a stronger line.

b The estimated line strength is computed using $N_{n} \propto g_{n} f_{n} \times 10^{-} \chi_{n} / k T$, where $f_{n}$ is the oscillator strength, $g_{n}$ is the statistical weight, $\chi_{n}$ is the excitation potential, and $T$ is the temperature. The values are normalized by dividing by the value for one of the strongest lines for each ion. This is usually the strongest line at that temperature, but in some cases (for example, Ca II) the strongest line is so much stronger that the normalization is not relevant. In that case, we chose the second or third strongest line (see Section 3.1).

found near 1.12, 1.21, 1.50, and $1.65 \mu \mathrm{m}$ are probably formed by local flux increasing on both sides of an apparent minimum rather than by absorption at that location.

Another example can be seen in Figure 10, where the entire region from 0.9 to $1.3 \mu \mathrm{m}$ displays a distinct change from a concave bend below a straight line in the epoch $+0 \mathrm{~d}$ to $+4 \mathrm{~d}$, to a nearly straight pattern from $+4 \mathrm{~d}$ to $+10 \mathrm{~d}$, and finally to a convex bulge above a straight line after $+10 \mathrm{~d}$ that continues in later epochs.

This group exhibits a reduced continuum level from 1.2 to $1.5 \mu \mathrm{m}$ as compared with the earlier spectra. Figures 9, 10, 14 , and 15 clearly show this effect. This depressed continuum level is due to the region having fewer blends of iron group lines compared to adjacent wavelengths (Wheeler et al. 1998). Note that the spectrum of SN 2001bf (+4d) does not exhibit any deficit in this region, even though the epoch is estimated to be only 1 day from that of SN 2000dk (+5d) in which the effect is pronounced.

We note that the notch found at $1.65 \mu \mathrm{m}$ between two emission peaks, occurs at approximately the same wavelength as the $\mathrm{Mg} / \mathrm{Si} / \mathrm{Co}$ absorption in earlier spectra, but the features are probably not related. The spectrum of SN $2000 \mathrm{dk}$ at $+5 \mathrm{~d}$ is the oldest to show the strong notch indicative of $\mathrm{Mg}$ II absorption at this location and later spectra have a smoother, shallower depression in this wavelength region. The spectrum from SN $2000 \mathrm{dk}$ at $+5 \mathrm{~d}$ is also the oldest spectrum to show
Mg II absorption from lines at 0.9227 and $1.0927 \mu \mathrm{m}$ (see Figure 12).

The steep slope on the blue side of the peak found near $1.55 \mu \mathrm{m}$ defines the transition from partial to complete silicon burning. Due to the high optical depth, this feature is formed close to the outer edge of the $\mathrm{Fe} / \mathrm{Co} / \mathrm{Ni}$ core at a rest wavelength of $\sim 1.57 \mu \mathrm{m}$. Precise determination of the transition feature is not possible but velocities measured to a point approximately half way up the slope are found between 9900 and $12,100 \mathrm{~km} \mathrm{~s}^{-1}$ for spectra in this group obtained from +4 to +18 days after maximum light.

\subsubsection{Transitional Phase (Inner Region; $+37 d$ to $+75 d$ )}

Figure 16 shows seven spectra from our sample obtained between 37 and 75 days after $V_{\max }$. This group displays a lot of new structure relative to earlier NIR spectra and the features are remarkably similar from one spectrum to the next. The spectrum of SN 2004bv at +60d fits well with the others in this group although the spectrum from this $\mathrm{SN}$ at $-5 \mathrm{~d}$ was unusual (see Figure 12). Spectra obtained in LRS mode extend blueward of $0.80 \mu \mathrm{m}$. All others were obtained in SXD mode. For a detailed discussion of individual features from this group, please reference Appendix A.4.

Synopsis. This epoch is a transitional phase between the photospheric era and a true nebular phase. This is the same time frame in which unreddened SNe Ia have a very low dispersion 
Table 6

Estimated Line Strengths at 10,000 K in the Region 0.7-2.4 $\mu \mathrm{m}$

\begin{tabular}{|c|c|c|c|c|c|c|}
\hline Ion & $\begin{array}{c}\lambda_{\text {rest }}(\text { air }) \\
(\mu \mathrm{m})\end{array}$ & $\begin{array}{c}\text { No. of Lines } \\
\text { in Blend }\end{array}$ & $\log (g f)$ & $\begin{array}{l}\text { Excitation } \\
\text { Min. }(\mathrm{eV})\end{array}$ & $\begin{array}{c}\text { Line Strength } \\
5000 \mathrm{~K}^{\mathrm{b}}\end{array}$ & $\begin{array}{c}\text { Line Strength } \\
10,000 \mathrm{~K}^{\mathrm{b}}\end{array}$ \\
\hline He I & 1.0830 & 3 & -0.047 & 19.82096 & 15.1 & 6.0 \\
\hline He I & 2.0581 & 1 & -0.424 & 20.61718 & 1.000 & 1.000 \\
\hline He I & 0.7065 & 3 & -0.460 & 20.96551 & 0.410 & 0.614 \\
\hline $\mathrm{C}_{\mathrm{I}}$ & 1.0693 & 7 & 0.348 & 7.48830 & 1.000 & 1.000 \\
\hline $\mathrm{C}_{\mathrm{I}}$ & 0.9093 & 2 & 0.142 & 7.48830 & 0.622 & 0.622 \\
\hline $\mathrm{C}_{\mathrm{I}}$ & 0.9406 & 1 & 0.225 & 7.68529 & 0.477 & 0.599 \\
\hline $\mathrm{C}_{\mathrm{I}}$ & 1.1754 & 2 & 0.661 & 8.64774 & 0.139 & 0.535 \\
\hline $\mathrm{C}_{\mathrm{I}}$ & 1.7325 & 8 & 1.030 & 9.70241 & 0.028 & 0.368 \\
\hline $\mathrm{C}_{\mathrm{I}}$ & 1.6890 & 1 & 0.568 & 9.00319 & 0.049 & 0.286 \\
\hline $\mathrm{C}_{\mathrm{I}}$ & 1.4543 & 1 & -0.110 & 7.68529 & 0.221 & 0.277 \\
\hline $\mathrm{C}_{\mathrm{I}}$ & 1.1330 & 1 & 0.280 & 8.53767 & 0.075 & 0.253 \\
\hline$\overline{\mathrm{C}_{\text {II }}}$ & 0.6580 & 2 & 0.118 & 14.44980 & 88,393 & 253 \\
\hline $\mathrm{C}_{\text {II }}$ & 0.7235 & 3 & 0.330 & 16.33422 & 1,815 & 46.3 \\
\hline $\mathrm{C}_{\text {II }}$ & 0.9903 & 3 & 1.010 & 20.95206 & 0.192 & 1.000 \\
\hline $\mathrm{C}_{\text {II }}$ & 1.8905 & 2 & 0.258 & 19.49586 & 1.000 & 0.959 \\
\hline $\mathrm{C}_{\text {II }}$ & 1.7846 & 3 & 0.550 & 20.15184 & 0.427 & 0.878 \\
\hline $\mathrm{C}_{\text {II }}$ & 0.9230 & 3 & 0.620 & 20.84618 & 0.100 & 0.461 \\
\hline$\overline{\mathrm{OI}_{\mathrm{I}}}$ & 0.7773 & 3 & 0.324 & 9.14671 & 1.000 & 1.000 \\
\hline $\mathrm{O}_{\mathrm{I}}$ & 0.8446 & 3 & 0.170 & 9.52201 & 0.294 & 0.454 \\
\hline $\mathrm{O}_{\mathrm{I}}$ & 0.9264 & 9 & 0.690 & 10.74166 & 0.057 & 0.365 \\
\hline $\mathrm{O}_{\mathrm{I}}$ & 1.1290 & 9 & 0.500 & 10.98960 & 0.021 & 0.177 \\
\hline $\mathrm{O}_{\mathrm{I}}$ & 1.8021 & 18 & 0.880 & 12.07943 & 0.004 & 0.120 \\
\hline O I & 0.7990 & 6 & 0.280 & 10.98960 & 0.013 & 0.106 \\
\hline$\overline{\mathrm{Mg} \text { II }}$ & 0.9227 & 2 & 0.270 & 8.65529 & 1.000 & 1.000 \\
\hline $\mathrm{Mg}$ II & 0.7890 & 3 & 0.650 & 10.00000 & 0.106 & 0.504 \\
\hline $\mathrm{Mg}$ II & 1.0927 & 3 & 0.020 & 8.86425 & 0.346 & 0.441 \\
\hline $\mathrm{Mg}$ II & 1.0092 & 3 & 1.020 & 11.63047 & 0.006 & 0.178 \\
\hline $\mathrm{Mg}_{\text {II }}$ & 0.8228 & 2 & 0.030 & 10.00000 & 0.025 & 0.121 \\
\hline $\mathrm{Mg}$ II & 0.9632 & 3 & 0.660 & 11.56982 & 0.003 & 0.083 \\
\hline $\mathrm{Mg}$ II & 1.8606 & 9 & 1.220 & 12.85873 & 0.001 & 0.068 \\
\hline $\mathrm{Mg}$ II & 1.6787 & 3 & 0.730 & 12.08542 & 0.001 & 0.054 \\
\hline $\mathrm{Mg}_{\text {II }}$ & 2.1369 & 1 & 0.390 & 11.50533 & 0.002 & 0.048 \\
\hline$\overline{\mathrm{Si} \text { II }}$ & 0.6355 & 2 & 0.297 & 8.12157 & 10,126 & 49.6 \\
\hline Si II & 0.9413 & 3 & 0.980 & 12.84018 & 1.000 & 1.000 \\
\hline Si II & 1.6930 & 2 & 0.350 & 12.14781 & 1.169 & 0.524 \\
\hline Si II & 0.7849 & 3 & 0.490 & 12.52627 & 0.671 & 0.466 \\
\hline Si II & 1.1737 & 3 & 0.620 & 12.88100 & 0.397 & 0.416 \\
\hline Si II & 1.7183 & 3 & 0.890 & 14.10541 & 0.043 & 0.187 \\
\hline Si II & 1.3681 & 2 & 0.130 & 12.88100 & 0.128 & 0.135 \\
\hline Si II & 2.1967 & 3 & 0.680 & 14.13227 & 0.025 & 0.112 \\
\hline$\overline{\mathrm{Si} \text { III }}$ & 0.9324 & 1 & -0.120 & 20.55363 & 7,223 & 41.6 \\
\hline Si III & 1.2523 & 3 & 0.500 & 24.99684 & 0.094 & 1.000 \\
\hline Si III & 0.7465 & 6 & 0.360 & 24.99684 & 0.068 & 0.724 \\
\hline Si III & 1.2601 & 3 & 0.160 & 24.99662 & 0.043 & 0.457 \\
\hline $\mathrm{Si}_{\text {III }}$ & 1.1341 & 3 & 0.430 & 25.56430 & 0.021 & 0.441 \\
\hline Si III & 0.9800 & 1 & 0.230 & 25.33550 & 0.023 & 0.363 \\
\hline Si III & 0.8266 & 6 & 0.830 & 26.65707 & 0.004 & 0.311 \\
\hline Si III & 0.7612 & 1 & 0.660 & 26.60039 & 0.003 & 0.225 \\
\hline Si III & 1.5015 & 1 & 0.030 & 25.77484 & 0.005 & 0.137 \\
\hline Si III & 0.9375 & 1 & -0.490 & 25.33550 & 0.004 & 0.069 \\
\hline Si III & 0.7729 & 2 & -0.680 & 24.99669 & 0.006 & 0.066 \\
\hline Si III & 1.0526 & 1 & -2.950 & 20.55363 & 1.000 & 0.062 \\
\hline$\overline{S_{\text {II }}}$ & 0.8315 & 1 & -0.470 & 14.06839 & 1.000 & 1.000 \\
\hline$S_{\text {II }}$ & 0.7967 & 1 & -0.820 & 14.00342 & 0.519 & 0.482 \\
\hline$S_{\text {II }}$ & 0.7590 & 1 & -0.880 & 14.23489 & 0.264 & 0.321 \\
\hline$S_{\text {II }}$ & 0.7721 & 1 & -0.990 & 14.29456 & 0.179 & 0.232 \\
\hline $\mathrm{S}_{\text {II }}$ & 0.8855 & 2 & -1.110 & 14.16002 & 0.185 & 0.206 \\
\hline $\mathrm{S}_{\text {II }}$ & 1.4501 & 2 & -0.216 & 16.59204 & 0.005 & 0.096 \\
\hline $\mathrm{S}_{\text {II }}$ & 1.3529 & 1 & -0.425 & 16.53575 & 0.004 & 0.063 \\
\hline$\overline{\mathrm{Ca}} \mathrm{II}$ & 0.8538 & 2 & -0.362 & 1.70005 & 13,944 & 40.3 \\
\hline Ca II & 0.8662 & 1 & -0.623 & 1.69252 & 7,780 & 22.3 \\
\hline
\end{tabular}




\begin{tabular}{|c|c|c|c|c|c|c|}
\hline \multicolumn{7}{|c|}{$\begin{array}{c}\text { Table } 6 \\
\text { (Continued) }\end{array}$} \\
\hline Ion & $\begin{array}{c}\lambda_{\text {rest }}(\text { air }) \\
(\mu \mathrm{m})\end{array}$ & $\begin{array}{l}\text { No. of Lines } \\
\text { in Blend }\end{array}$ & $\log (g f)$ & $\begin{array}{l}\text { Excitation } \\
\text { Min. (eV) }\end{array}$ & $\begin{array}{l}\text { Line Strength } \\
5000 \mathrm{~K}^{\mathrm{b}}\end{array}$ & $\begin{array}{c}\text { Line Strength } \\
10,000 \mathrm{~K}^{\mathrm{b}}\end{array}$ \\
\hline$\overline{\mathrm{Ca} \text { II }}$ & 0.8921 & 3 & 0.729 & 7.05003 & 0.696 & 1.000 \\
\hline $\mathrm{Ca}$ II & 1.1839 & 1 & 0.300 & 6.46831 & 1.000 & 0.731 \\
\hline $\mathrm{Ca}$ II & 0.8249 & 2 & 0.621 & 7.51535 & 0.184 & 0.454 \\
\hline $\mathrm{Ca}$ II & 1.1950 & 1 & 0.000 & 6.46831 & 0.501 & 0.367 \\
\hline $\mathrm{Ca}$ II & 0.9891 & 3 & 0.943 & 8.43855 & 0.045 & 0.327 \\
\hline $\mathrm{Ca}$ II & 0.8202 & 1 & 0.315 & 7.50564 & 0.093 & 0.227 \\
\hline $\mathrm{Ca}$ II & 1.8850 & 2 & 0.878 & 9.01809 & 0.010 & 0.144 \\
\hline $\mathrm{Ca}$ II & 0.9931 & 1 & 0.072 & 7.51535 & 0.052 & 0.128 \\
\hline$\overline{M n ~ I I ~}$ & 0.7414 & 3 & -2.202 & 3.70608 & 1.000 & 1.000 \\
\hline Mn II & 0.8695 & 1 & 0.577 & 9.24489 & 0.002 & 0.971 \\
\hline Mn II & 0.8769 & 1 & 0.491 & 9.24489 & 0.001 & 0.797 \\
\hline Mn II & 0.9486 & 2 & 0.491 & 9.46988 & 0.001 & 0.614 \\
\hline Mn II & 0.8820 & 1 & 0.347 & 9.24489 & 0.001 & 0.572 \\
\hline Mn II & 0.9447 & 1 & -2.389 & 4.06545 & 0.282 & 0.428 \\
\hline Mn II & 0.7342 & 2 & -2.713 & 3.70979 & 0.306 & 0.307 \\
\hline Mn II & 0.9388 & 2 & -2.554 & 4.07405 & 0.189 & 0.290 \\
\hline Mn II & 0.7221 & 3 & 0.768 & 10.67048 & 0.000 & 0.288 \\
\hline Mn II & 0.9444 & 1 & 0.129 & 9.46988 & 0.000 & 0.267 \\
\hline Mn II & 0.8110 & 1 & -2.002 & 5.37762 & 0.033 & 0.228 \\
\hline Mn II & 1.5401 & 3 & 0.237 & 9.86600 & 0.000 & 0.216 \\
\hline Fe II & 0.8288 & 1 & 0.693 & 9.65426 & 0.003 & 1.000 \\
\hline $\mathrm{Fe}$ II & 0.9297 & 1 & 0.412 & 9.65426 & 0.001 & 0.524 \\
\hline Fe II & 0.7712 & 1 & -2.543 & 3.90368 & 1.000 & 0.460 \\
\hline $\mathrm{Fe}$ II & 0.9998 & 1 & -1.826 & 5.48450 & 0.133 & 0.382 \\
\hline $\mathrm{Fe}$ II & 0.9095 & 1 & 0.269 & 9.65426 & 0.001 & 0.377 \\
\hline $\mathrm{Fe}$ II & 0.9183 & 2 & 0.245 & 9.70089 & 0.001 & 0.338 \\
\hline Fe II & 0.7462 & 1 & -2.734 & 3.89187 & 0.662 & 0.300 \\
\hline $\mathrm{Fe}_{\text {II }}$ & 1.0500 & 2 & -1.997 & 5.54914 & 0.077 & 0.239 \\
\hline $\mathrm{Fe}_{\text {II }}$ & 1.0863 & 1 & -2.121 & 5.58957 & 0.053 & 0.172 \\
\hline$\overline{\mathrm{Fe}} \mathrm{III}$ & 0.7265 & 1 & -1.708 & 14.62496 & 0.501 & 1.000 \\
\hline Fe III & 0.7261 & 1 & -2.910 & 13.13443 & 1.000 & 0.354 \\
\hline $\mathrm{Fe}$ III & 0.9124 & 1 & -2.577 & 14.62496 & 0.068 & 0.135 \\
\hline Fe III & 1.2786 & 1 & 0.723 & 23.67243 & 0.000 & 0.007 \\
\hline Fe III & 1.2039 & 2 & 0.663 & 23.61137 & 0.000 & 0.007 \\
\hline Fe III & 1.6722 & 1 & 0.235 & 22.87010 & 0.000 & 0.006 \\
\hline Fe III & 1.2955 & 2 & 0.602 & 23.67243 & 0.000 & 0.006 \\
\hline Co II & 0.8342 & 2 & 0.753 & 10.41714 & 0.003 & 1.000 \\
\hline Co II & 0.8737 & 1 & 0.663 & 10.41714 & 0.002 & 0.813 \\
\hline Co II & 1.7772 & 1 & -2.087 & 5.04595 & 1.000 & 0.736 \\
\hline Co II & 0.8582 & 1 & 0.598 & 10.48815 & 0.002 & 0.644 \\
\hline Co II & 0.9070 & 1 & 0.433 & 10.41714 & 0.001 & 0.479 \\
\hline Co II & 1.7462 & 1 & -2.284 & 5.12265 & 0.532 & 0.428 \\
\hline Co II & 0.8803 & 2 & 0.521 & 10.70637 & 0.001 & 0.419 \\
\hline Co II & 2.2205 & 1 & -2.374 & 5.04595 & 0.516 & 0.380 \\
\hline Co II & 0.8512 & 2 & 0.426 & 10.61330 & 0.001 & 0.375 \\
\hline Co II & 2.4596 & 1 & -2.416 & 5.12265 & 0.392 & 0.316 \\
\hline Co II & 2.3613 & 1 & -2.491 & 5.17524 & 0.292 & 0.250 \\
\hline
\end{tabular}

Notes.

${ }^{a}$ If more than one line is included in the blend, the indicated wavelength is a mean value, weighted by oscillator strength. Some lines have been omitted from the table if their rest wavelengths are very close to that of a stronger line.

b The estimated line strength is computed using $N_{n} \propto g_{n} f_{n} \times 10^{-\chi_{n} / k T}$, where $f_{n}$ is the oscillator strength, $g_{n}$ is the statistical weight, $\chi_{n}$ is the excitation potential, and $T$ is the temperature. The values are normalized by dividing by the value for one of the strongest lines for each ion. This is usually the strongest line at that temperature, but in some cases (for example, Ca II) the strongest line is so much stronger that the normalization is not relevant. In that case, we chose the second or third strongest line (see Section 3.1).

for $B-V$ colors (Lira 1996). The common assumption about this era is that expansion has reduced the opacity beyond the point where a distinct photosphere can be defined and individual absorption features can no longer be produced. Recent work by Branch et al. (2008) suggests spectra from this epoch can be modeled by absorption features from permitted lines.
The apparent emission peaks at 1.60, 1.75, 2.15, 2.25, and $2.35 \mu \mathrm{m}$ are the result of increased flux due to line blending from iron group elements as explained in Section 5.3. The radius of optical depth unity has increased at these wavelengths producing a larger effective radiation area and an increased flux. 


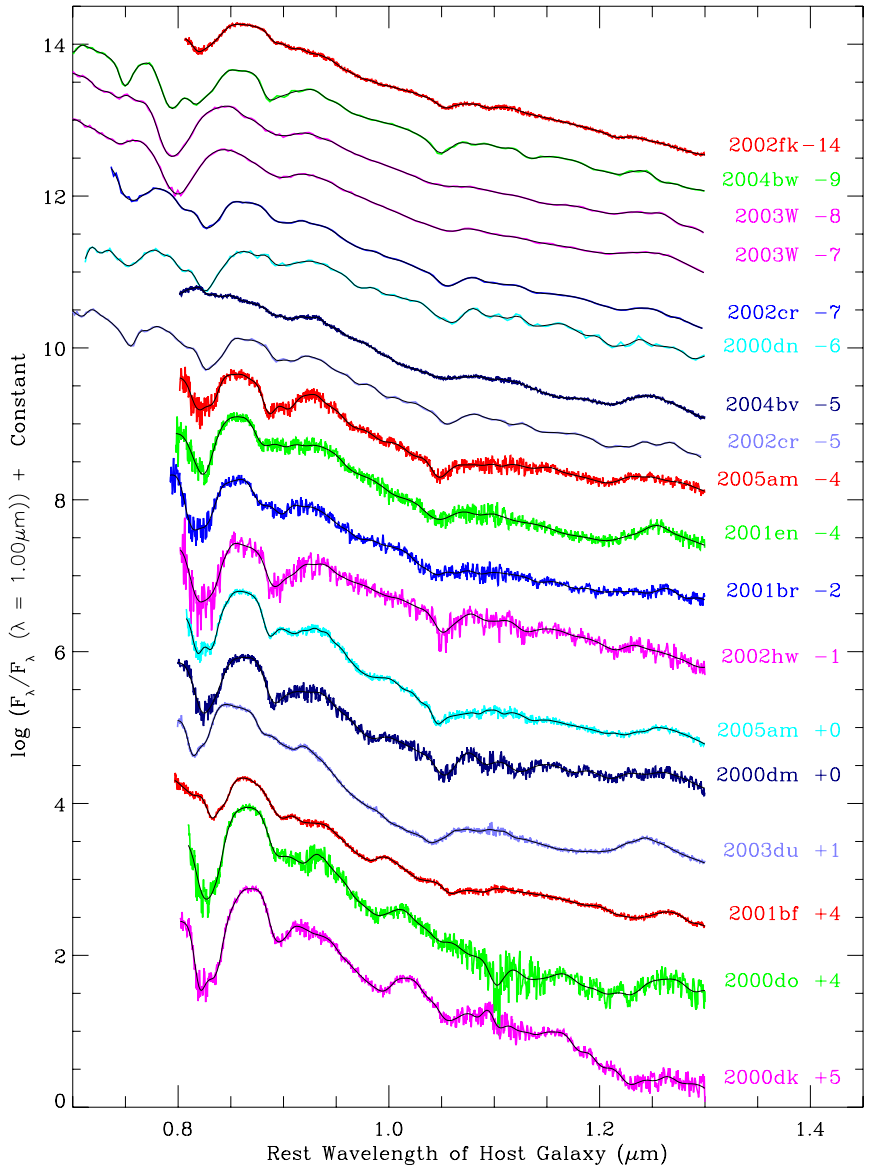

Figure 12. Eighteen spectra from the Photospheric Phase of SNe Ia obtained between $-14 \mathrm{~d}$ and $+5 \mathrm{~d}$ relative to $V_{\max }$. During this epoch, the photosphere is expected to be in the outer layers of the $\mathrm{SN}$ and the line-forming regions will be close to the photosphere. The figure displays only the wavelength region $0.65-1.35 \mu \mathrm{m}$ for detail. The complete spectra are found in Figure 10. A general discussion of features detected during the Photospheric Phase can be found in Section 3.1.2 and a more detailed analysis in found in Appendix A.2.

(A color version of this figure is available in the online journal.)

Fe II lines at $0.7712,0.9998$, and $1.0863 \mu \mathrm{m}$ appear in absorption beginning about 30 days after maximum light. In spectra from our sample obtained before $+37 \mathrm{~d}$, the $0.9998 \mu \mathrm{m}$ line is the only Fe II line detected, although the calculated line strengths suggest that lines at 0.7462, 0.7712, 1.0500, and $1.0863 \mu \mathrm{m}$ lines should also be detected.

Co II lines are clearly detected from lines at 1.7772, 1.7462, $2.2205,2.4596$, and $2.3613 \mu \mathrm{m}$ at velocities near $9000 \mathrm{~km} \mathrm{~s}^{-1}$. Co II is found in wavelength regions where a pseudophotosphere is created at extended radii (1.5-1.85 and 2.05-2.45 $\mu \mathrm{m})$. Strong absorptions are detected from several other Co II lines.

Figure 17 shows evidence for Co II and Fe II lines in 13 spectra from our sample obtained between $+14 \mathrm{~d}$ and $+75 \mathrm{~d}$ with respect to $V_{\max }$. The figure includes two additional spectra from $\mathrm{SNe}$ 1998aq and 1998bu (plotted in yellow) that were obtained by R. J. Rudy \& R. Puetter (2007, private communication) and have not been previously published. These spectra are added to verify the consistency of our results. The positions of strong Co II and Fe II lines drawn in the figure to indicate $11,000 \mathrm{~km} \mathrm{~s}^{-1}$ at the top spectrum and $6000 \mathrm{~km} \mathrm{~s}^{-1}$ for the bottom spectrum. This corresponds to the reduction in measured velocities during this epoch.

The steep increase in flux found near $1.55 \mu \mathrm{m}$ is used by some models to define the transition from partial to complete silicon burning. This feature is well defined up to about $+60 \mathrm{~d}$. As shown in Figure 16, the spectrum from SN 2004bv at +60 displays this feature but it is smeared out in the spectrum of SN $2001 \mathrm{fe}$, also obtained at $+60 \mathrm{~d}$.

\section{RESULTS}

\subsection{Uniformity of Spectral Evolution}

Figures 9, 10, 12, 14, and 16 reveal that the development of spectral features with time is very consistent throughout the sample. The data show that spectra obtained from normal SNe Ia at close to the same age have similar but not identical characteristics. There are many small variations in features between adjacent spectra and a few instances where one spectrum displays a prominent feature that is absent or weak in other spectra with similar relative ages. Nevertheless, within uncertainties introduced by variations in data quality and dating methods, the progress of feature development and overall shape is remarkably coherent from spectrum to spectrum in our sample. That means that NIR spectra from different objects can be directly compared and arranged sequentially to mimic a time series. Even spectroscopically unusual SNe Ia such as 2004bv and 2004da, which are missing some of the features found in most other $\mathrm{SNe}$ Ia, fit well with the general profile of other spectra obtained at similar epochs. The agreement in spectral features from so many different $\mathrm{SNe}$ Ia eliminates the possibility that observations of finite line-forming regions (Section 4.2) are due to clumps of material.

We test this hypothesis by comparing our "snapshot" spectra from separate $\mathrm{SNe}$ Ia to an excellent time series of NIR spectra obtained by Hamuy et al. (2002) from SN 1999ee. Five spectra from this series obtained between $-11 \mathrm{~d}$ and $+13 \mathrm{~d}$ are displayed with 24 spectra from our sample in Figure 18. Our data include a series of four spectra from SN 2005am ( $-4 \mathrm{~d}$ to $+14 \mathrm{~d}$ ). The progression of feature development in a time series of spectra obtained from individual SNe Ia matches well with the sequence of spectra obtained from diverse objects. It is clear that spectral development of normal SNe Ia in the NIR is very consistent.

Although the overall NIR spectral evolution for SNe Ia is uniform in basic shape and features, there is obviously a great deal of diversity in the class of "normal" SNe Ia. For example, our most confident line identification (apart from the Ca II triplet) is $\mathrm{Mg}$ II at $1.0927 \mu \mathrm{m}$. Yet spectra obtained within a day or two of each other sometimes exhibit dramatically different depths and widths for the absorption features produced by this line (see Figures 12 and 13). Other examples of diversity include the anomalous velocities of SNe 2002cr and 2001bf, the weak features in the $-4 d$ spectrum from SN 2004bv, the variations in size and shape of the notch at $1.6 \mu \mathrm{m}$ over a range of epochs, and the vagaries of the unidentified bump at $1.25 \mu \mathrm{m}$. The presence of such variability provides motivation for further research and data collection efforts. Despite these variations in individual features, the overall spectral shape and the general behavior of most features permits placing a spectrum in temporal order within a few days relative to $V_{\max }$.

\subsection{Measured Doppler Velocities}

The Doppler shift from the rest wavelength of a line measures the velocity of the absorbing material along the line of sight to the observer. As discussed in Section 5.3, when the photosphere is close to the line-forming region, the observed Doppler-shift of the absorption minimum of the spectral feature corresponds 

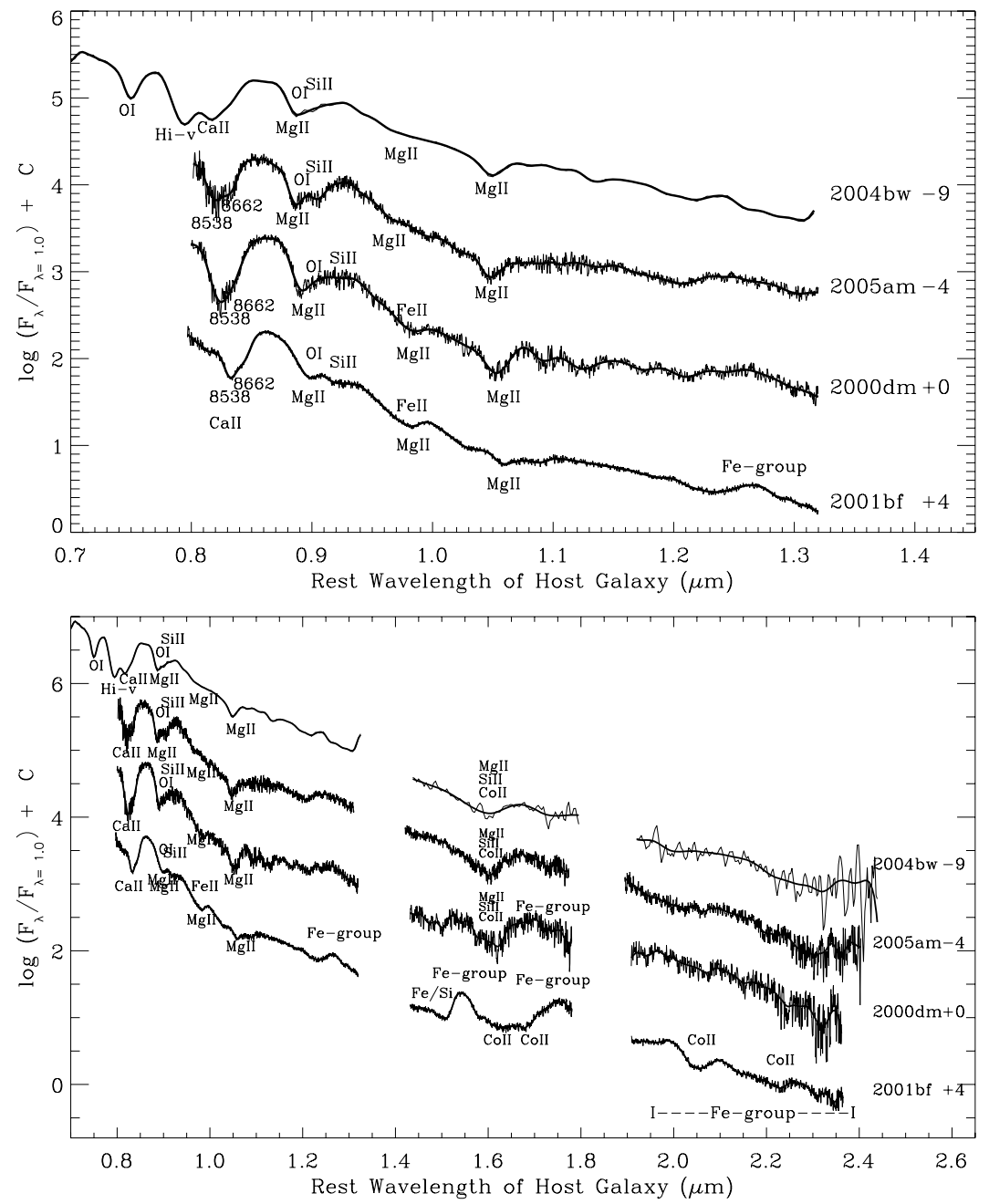

Figure 13. Four representative spectra from the Photospheric Phase of spectral development with features labeled. The spectra are from SNe 2004bw (LRS obtained at $-9 \mathrm{~d}$ ), 2005am (SXD obtained at $-4 \mathrm{~d}$ ), 2000dm (SXD obtained at $+0 \mathrm{~d}$ ), and 2001bf (SXD obtained at $+4 \mathrm{~d}$ ). The top panel displays only the wavelength region $0.65-1.35 \mu \mathrm{m}$ for detail, while the bottom panel contains the complete spectra. A general discussion of these features can be found in Section 3.1.2 and a more detailed analysis in found in Appendix A.2.

to the velocity of the photosphere. At later times expansion moves the line-forming region away from the photosphere. If there is a distinct discontinuity in the composition of the lineforming region, then a boundary of the absorbing layer for a particular ion is clearly defined and the velocity of the absorption minimum for an ion is determined by the inner edge of this layer. The absorption feature will decrease in strength as the column density drops, but the velocity of the absorption minimum will no longer change until the ion is undetectable.

Table 4 and Figure 19 show the measured Doppler velocities for $\mathrm{OI}_{\mathrm{I}}, \mathrm{Mg}$ II, Si II, S I, Ca II, and Fe II. Distinct minimum velocities, as measured from the absorption minima of spectral features, exist for all of these intermediate-mass ions. These velocity minima correspond to the location of the inner edge of a layer of line-forming matter in velocity space and physical space (Section 5.3). Figure 19 shows that the line-forming regions from two $\mathrm{O}$ I lines and three $\mathrm{Mg}$ II lines occupy similar velocity spaces at $\approx 10,000-15,000 \mathrm{~km} \mathrm{~s}^{-1}$. The velocity spaces for Si II and $\mathrm{S}$ I are slightly lower but overlap the $\mathrm{O}$ I and $\mathrm{Mg}$ II region at $\approx 8000-12,000 \mathrm{~km} \mathrm{~s}^{-1}$. The Ca II velocities are measured for the IR triplet and may not be produced by fresh burning products (Section 5.3). This may account for the fact that minimum velocities for Ca II are not as consistent as they are for some other species. Fe II appears to have no minimum velocity as the detected velocity descends from about $12,000 \mathrm{~km} \mathrm{~s}^{-1}$ at 10 days before maximum brightness to about $2000 \mathrm{~km} \mathrm{~s}^{-1}$ at 20 days after maximum.

The distribution of burning products and radially stratified chemical structure is consistent with the explosion occurring in a $\mathrm{C} / \mathrm{O}$ WD with a radial density gradient and lower densities on the outside (Section 5.2). The data appear to eliminate the possibility of large-scale mixing of lower mass elements to the center or Fe-group elements to the extreme outer layers.

\subsection{Nondetection of Carbon}

Figure 20 displays the 18 spectra from Figure 12 with the locations of strong $\mathrm{C}_{\mathrm{I}}$ lines marked at Doppler velocities of $11,000 \mathrm{~km} \mathrm{~s}^{-1}$. The strongest lines at $5000 \mathrm{~K}$, as listed in Table 5, are marked in red in the figure and the weaker lines appear in black. Small features appear in some spectra near the expected location for lines from $0.9406,1.1330$, and $1.1754 \mu \mathrm{m}$, but if these lines produce absorption features then the strongest lines at 1.0693 and $0.9093 \mu \mathrm{m}$ should be detected and they are not. The possible feature for the $0.9406 \mu \mathrm{m}$ line is more likely attributed to Si II at $0.9413 \mu \mathrm{m}$. (A complete discussion of this group of spectra is found in Section 3.1.2 and Appendix A.2.)

Carbon is not detected in these data, a result that agrees with previous results with NIR data establishing very low limits on 


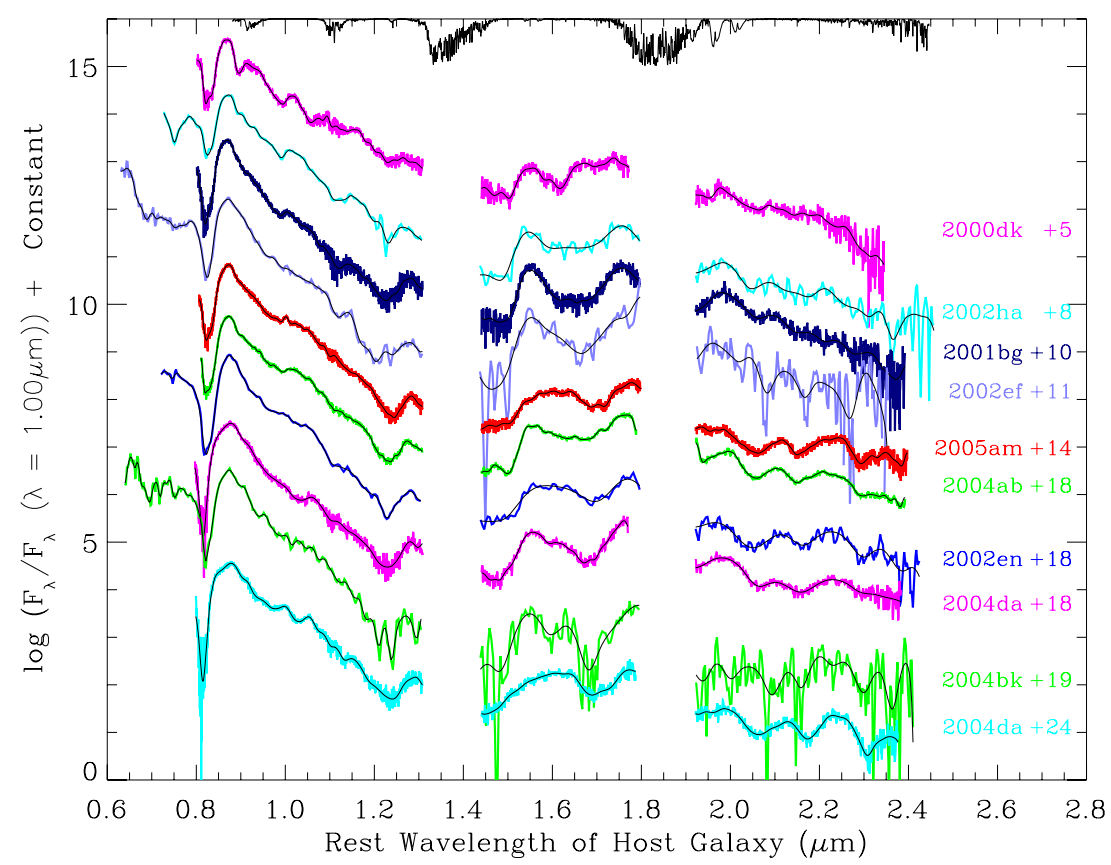

Figure 14. Ten spectra from the Extended Photospheric Phase of SNe Ia obtained between $+4 \mathrm{~d}$ and $+24 \mathrm{~d}$ with respect to $V_{\max }$. During the phase represented by this group, the shape of the continuum becomes influenced by line-blanketing from Fe-group lines which extends the effective photospheric radius (see Section 5.3). A general discussion of features detected during the Extended Photospheric Phase can be found in Section 3.1.3 and a more detailed analysis is found in Appendix A.3. (A color version of this figure is available in the online journal.)

carbon abundance in SNe Ia (Marion et al. 2006). Similar results have been obtained using $\mathrm{C}_{\text {II }}$ lines in optical spectra (Tanaka et al. 2008), but the much higher excitation potentials for $\mathrm{C}_{\text {II }}$ lines in the NIR make it unlikely that $\mathrm{C}_{\text {II }}$ will be detectable in these NIR data. It is clear however that NIR data show that carbon burning products, $\mathrm{O}$ and $\mathrm{Mg}$, are plentiful in the outer layers and that $\mathrm{C}$ is absent. The line strengths for $\mathrm{C}$ and $\mathrm{O}$ will be similar for the same ionization stages, and if $\mathrm{O}_{\mathrm{I}}$ in the data indicates unburned material, then $\mathrm{C}_{\mathrm{I}}$ should also be present in detectable amounts (Marion et al. 2006). This result suggests that the entire progenitor is burned in the explosion.

\section{SNE IA PHYSICS}

\subsection{Generic Model for SNe Ia}

Many years of optical observations support the widely accepted model that $\mathrm{SNe}$ Ia are the result of a thermonuclear explosion in a C/O WD (Hoyle \& Fowler 1960). The WD mass at the time of explosion is expected to be near the Chandrasekhar $\operatorname{limit}\left(M_{\mathrm{Ch}} \approx 1.4 M_{\odot}\right)$. The progenitor WD begins with a mass well below $M_{\mathrm{Ch}}$ and may grow by accreting matter from a red giant or main-sequence binary companion by Roche lobe overflow. Alternative models for SNe Ia create progenitors with an He star or another WD as the donor companion. These evolutionary tracks will also produce a C/O WD near $M_{\mathrm{Ch}}$. Burning of accreted $\mathrm{H}$ or $\mathrm{He}$ on the surface of the WD converts it to $\mathrm{C}$ and $\mathrm{O}$ in approximately equal abundance (Abia et al. 2001). Since the WD is supported against gravitational collapse by the degenerate electron gas, the final structure does not depend on the history of the progenitor. Given the similar quantity and composition of materials, it is reasonable to assume that all progenitors will explode with similar properties.

The primary fuel in the combustion of SNe Ia is carbon and oxygen which produces an ash that consists predominately of elements between silicon and nickel. The composition of the final burning products does not affect the energy production because very little additional binding energy is released in the final transition from $\mathrm{Si}$-group to $\mathrm{Fe}$-group elements. If a $\mathrm{C} / \mathrm{O}$ WD progenitor with mass $\approx 1.4 M_{\odot}$ burns its entire mass to at least Si-group elements, $\approx 2 \times 10^{51} \mathrm{erg}$ of nuclear energy will be produced. That is sufficient energy to overcome the gravitational binding energy of (5-7) $\times 10^{50}$ erg and provide the kinetic energy to accelerate the ejecta to observed expansion velocities (Höflich et al. 1993, 2002; Höflich 1995).

The results described in Section 4.3 suggest that the progenitor is almost entirely consumed in the explosion. The nearly complete burning of progenitors with very similar characteristics can explain why the total production of nuclear energy is approximately constant in $\mathrm{SNe}$ Ia.

\subsection{Chemical Structure of SNe Ia}

Optical spectra of SNe Ia reveal a range of elements produced by nuclear burning of carbon and oxygen at densities of $10^{6}-10^{9} \mathrm{~g} \mathrm{~cm}^{-3}$. To agree with observations of intermediatemass elements, burning conditions must exist in $\mathrm{SNe}$ Ia at densities less than $\approx 10^{7} \mathrm{~g} \mathrm{~cm}^{-3}$. Since the initial density of a C/ $\mathrm{O}$ WD near $M_{\mathrm{Ch}}$ is $\approx 10^{9} \mathrm{~g} \mathrm{~cm}^{-3}$, it is necessary for early burning to lift the WD in its gravitational potential causing it to expand and reduce the mean density (Höflich et al. 1998). The quantities and distribution of different burning products are determined by the relation between the hydrodynamical and nuclear timescales. The hydrodynamical timescale is $\approx 1 \mathrm{~s}$, but the nuclear rates vary with density. The range of densities implied by observations includes three distinct regions that produce different products from thermonuclear combustion (Höflich et al. 1993, 2002; Höflich 1995).

At densities greater than $10^{7} \mathrm{~g} \mathrm{~cm}^{-3}$, temperatures exceed $5 \times 10^{9} \mathrm{~K}$. Thermonuclear burning under these conditions proceeds uniformly to nuclear statistical equilibrium (NSE) and produces $\mathrm{Fe}$-peak elements $(\mathrm{Fe} / \mathrm{Co} / \mathrm{Ni})$. In the case that 

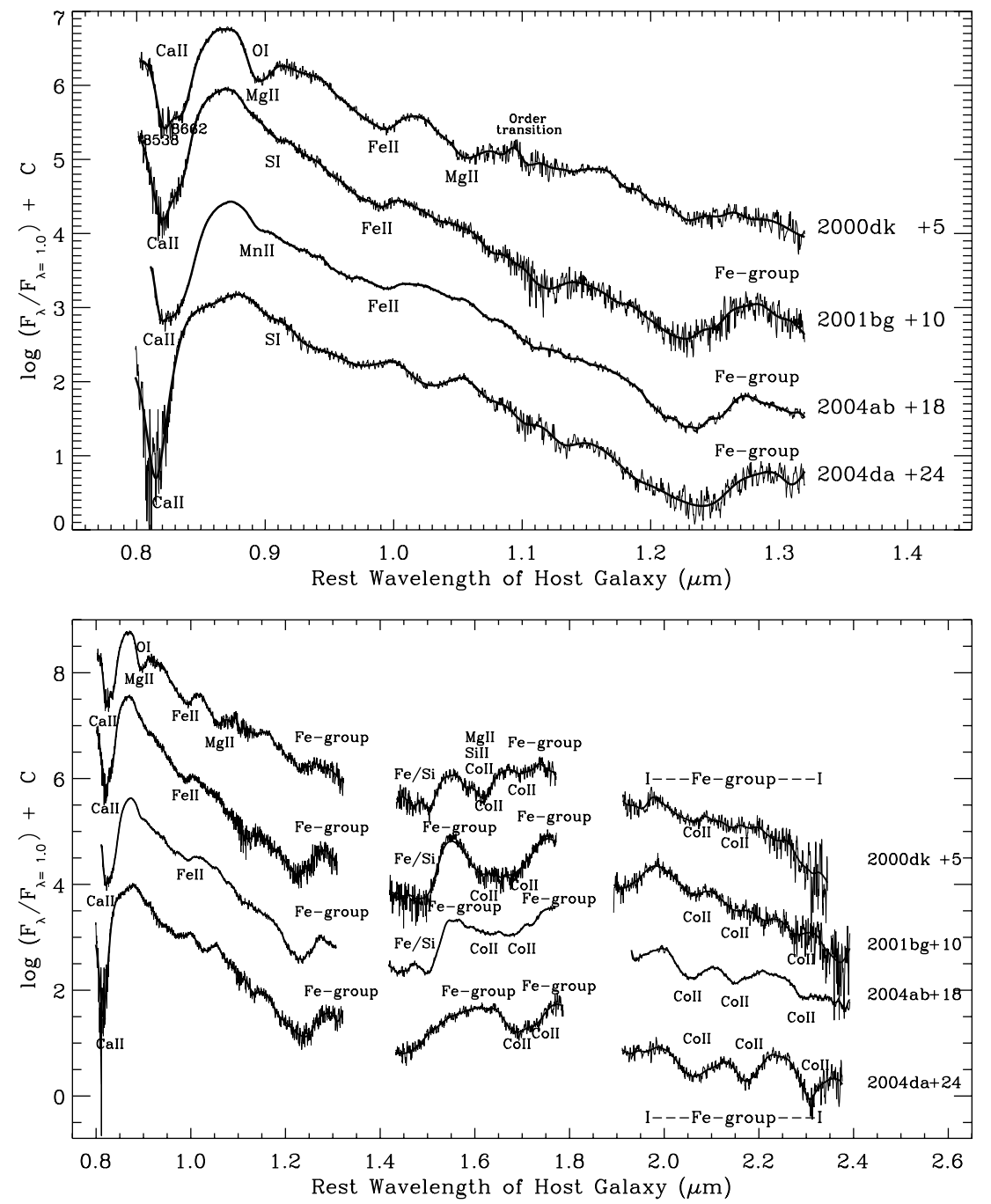

Figure 15. Four representative spectra from the Extended Photospheric Phase of spectral development with features labeled. The spectra are from SNe 2000dk (+5d), $2001 \mathrm{bg}(+10 \mathrm{~d})$, 2004ab (+18d), and 2004da (+24d). The top panel displays only the wavelength region $0.65-1.35 \mu \mathrm{m}$ for detail while the bottom panel contains the complete spectra. A general discussion of these features can be found in Section 3.1.3 and a more detailed analysis in found in Appendix A.3.

$\rho_{\text {burn }}>10^{9} \mathrm{~g} \mathrm{~cm}^{-3}$, electron capture results and stable isotopes are produced. For $10^{9} \mathrm{~g} \mathrm{~cm}^{-3}>\rho_{\text {burn }} \geqslant 10^{7} \mathrm{~g} \mathrm{~cm}^{-3}$, the final burning product is predominantly ${ }^{56} \mathrm{Ni}$. The existence of lowand intermediate-mass elements in $\mathrm{SNe}$ Ia requires the WD to experience a phase of expansion to reduce densities in the outer layers and prevent the entire WD from burning to Ni.

Intermediate-mass elements such as $\mathrm{Si}, \mathrm{S}$, and $\mathrm{Ca}$ are produced in a wide range of burning conditions $\left(10^{7} \mathrm{~g} \mathrm{~cm}^{-3}\right.$ $>\rho_{\text {burn }} \geqslant 4 \times 10^{6} \mathrm{~g} \mathrm{~cm}^{-3}$ and $5 \times 10^{9} \mathrm{~K}>T>3 \times 10^{9} \mathrm{~K}$ ). Similar abundance ratios for these elements are produced by both explosive $\mathrm{O}$ burning and incomplete Si burning. At the high end of this temperature and density range, silicon continues to burn after both carbon and oxygen are completely consumed, but the Si does not burn all the way to NSE.

In regions with the lowest burning temperatures and densities ( $\rho_{\text {burn }} \leqslant 4 \times 10^{6} \mathrm{~g} \mathrm{~cm}^{-3}$ and $T \leqslant 3 \times 10^{9} \mathrm{~K}$ ), explosive carbon burning occurs generating ash that consists primarily $\mathrm{O}, \mathrm{Mg}$, and Ne. The presence of these elements and the absence of $\mathrm{C}$ indicates that nuclear burning has occurred, since unburned regions of the $\mathrm{SN}$ will contain only $\mathrm{C}$ and $\mathrm{O}$ from the progenitor. Multiply ionized atoms from heavier elements may be found in the outer layers from primordial sources.

\subsection{Location of the Photosphere and Line-forming Region}

We define the photosphere as the region within the expanding envelope where continuum photons are produced at a given time and wavelength. The photospheric radius is not constant, but is a function of wavelength. The specific location is a function of opacity in the envelope and the energy deposition rate from radioactive decay. As expansion reduces the optical depth in the outer layers, sequentially deeper layers of explosion products are revealed. A time series of spectra will trace the chemical composition from the outer regions toward the core as the photosphere recedes in mass space. NIR observations are particularly effective for recording the chemical structure of SNe Ia because the optical depth for most lines is less in the NIR than it is at shorter wavelengths. As a result, a greater radial distance can be probed with each spectrum (Wheeler et al. 1998).

Absorption lines found in spectra from SNe Ia are formed in regions of expanding material that lie between the photosphere and the observer. These line-forming regions in $\mathrm{SNe}$ Ia are extended in radial space and hence in velocity space. To define the location of the line-forming region in radial space we use 


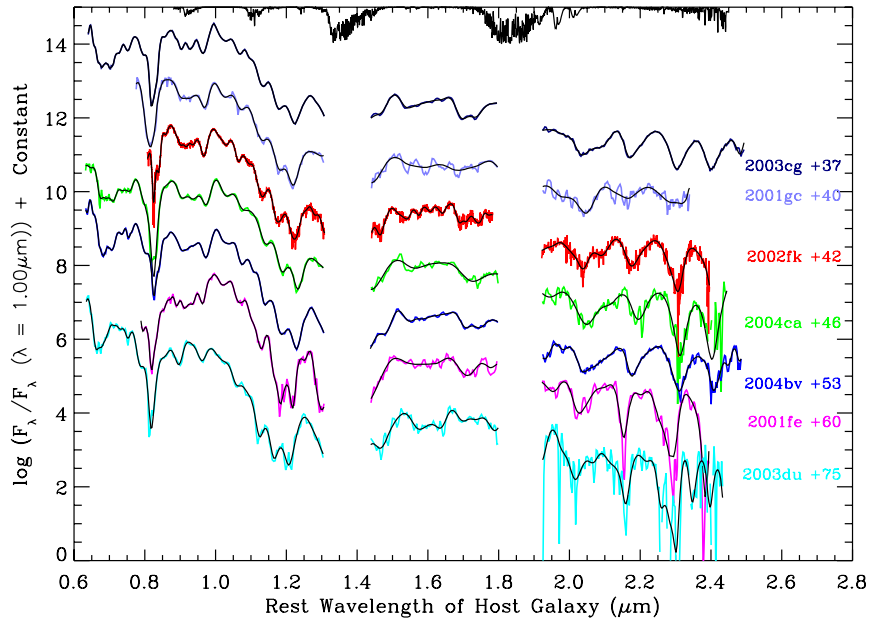

Figure 16. Seven spectra from the Transitional Phase SNe Ia obtained between $+37 \mathrm{~d}$ and $+75 \mathrm{~d}$ with respect to $V_{\max }$. This epoch is a transitional phase between the photospheric era and a true nebular phase. This group displays a lot of new structure relative to earlier NIR spectra and the features are remarkably similar from one spectrum to the next. A general discussion of features detected during the Co II Phase can be found in Section 3.1.4 and a more detailed analysis is found in Appendix A.4.

(A color version of this figure is available in the online journal.)

the Doppler shifts of the absorption components of individual spectral features to measure the expansion velocities. The radial extent of each line-forming region covers the full range of Doppler velocities between the absorption minimum and the detection limit in the blue wings of each absorption feature.

During the first few weeks after the explosion, high temperatures within the envelope ensure that the primary opacity source in the NIR is Thomson scattering with some contribution from free-free emission. In a rapidly expanding, scatteringdominated envelope, all lines contain an emission component due to a nonvanishing line source function whether or not they display an obvious P Cygni profile. Emission photons from each line will reach the observer from different parts of the expanding envelope with different observed velocities. These emission photons contaminate the absorption feature and make Doppler measurements unreliable between the rest wavelength of the line and the wavelength of the absorption minimum in each spectral feature.

During this phase, while the photosphere and line-forming region are close, the minimum observed Doppler velocity is determined by the location of the photosphere. Because expansion velocity is proportional to radius, as the photosphere moves deeper with time, progressively slower material is exposed in the line-forming region. As a result, the measured velocity at the absorption minimum for each line will diminish with time until there is insufficient abundance to produce a detectable signal. The measured range of velocities from the absorption minimum to the blue wing of the feature represents the physical location of the line-forming region in the ejecta.

In the case where the line-forming region containing a particular ion does not extend below a certain radius, continued expansion will separate the photosphere from the line-producing layer. The observed Doppler velocity in all spectra obtained after separation occurs will be the velocity at the inner edge of this layer, and the measured Doppler velocity at the absorption minimum will no longer change. The absorption feature will subsequently decrease in strength as the column density drops due to the expansion of the line-forming region. Since the

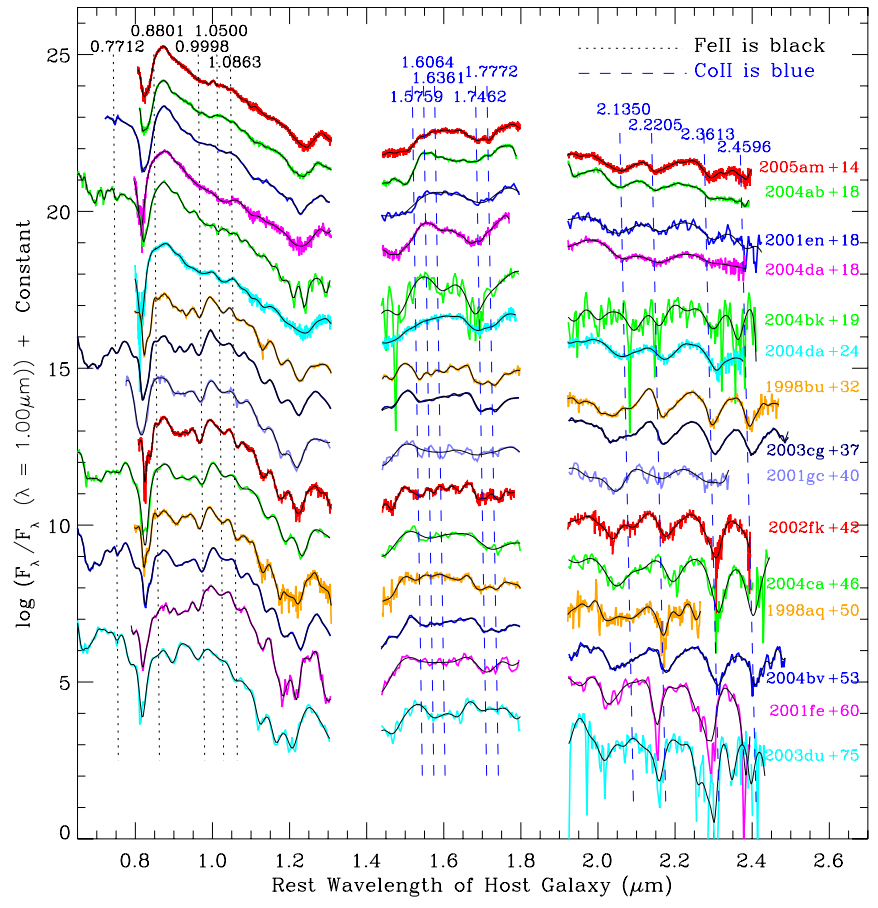

Figure 17. Fifteen spectra between $+14 d$ and $+75 d$ are presented with the positions of strong Co II and Fe II lines drawn in the figure so that the indicated velocities are $11,000 \mathrm{~km} \mathrm{~s}^{-1}$ for the top spectrum and $6000 \mathrm{~km} \mathrm{~s}^{-1}$ for the bottom spectrum. This corresponds to the measured velocities for features from spectra during this epoch. The data plotted in yellow (SNe 1998aq and 1998bu) were obtained by R. J. Rudy \& R. Puetter (2007, private communication) and have not been previously published. Co II lines are easily detected in all spectra in this group and Fe II lines begin to appear about 1 month post-maximum (see Sections 3.1.3 and 3.1.4).

(A color version of this figure is available in the online journal.)

photosphere is detached from the absorbing region, lines are formed far outside the last scattering radius for continuum photons and the photosphere appears to be nearly pointlike.

We note that during very early epochs $\left(\geqslant 10\right.$ days before $\left.V_{\max }\right)$ the envelope is actually cooler than it will soon become, when it warms due to increased energy deposition from radioactive decay. The cooler material has a lower optical depth, causing the photosphere to temporarily recede far enough toward the center that some of the intermediate-mass materials beneath the extreme outer layers are revealed. Doppler velocities are relatively low since the photosphere is closer to the center of mass and higher pressures in the interior near the photosphere will produce higher ionizations. Thus, multiple ionizations stages from intermediate-mass elements may be detected in a single spectrum due to the large range of radial distance probed by NIR spectra.

Later than approximately 10 days before $V_{\max }$, the expanding material continues to be heated by radioactive decay and the increasing temperature increases the opacity and moves the photosphere away from the center in both mass and velocity space. From this time forward, the photosphere begins the monotonic recession in mass and velocity space that characterizes spectral evolution during the next 15-20 days, as the data in this paper demonstrate.

A few days after $V_{\max }$, line-blanketing from thousands of Fe-group lines begins to dominate the spectrum at certain wavelength regions. The increase in total opacity moves the radius at which features are formed well above the receding photosphere formed by electron-scattering. A larger radius increases the effective area from which the observed flux is 


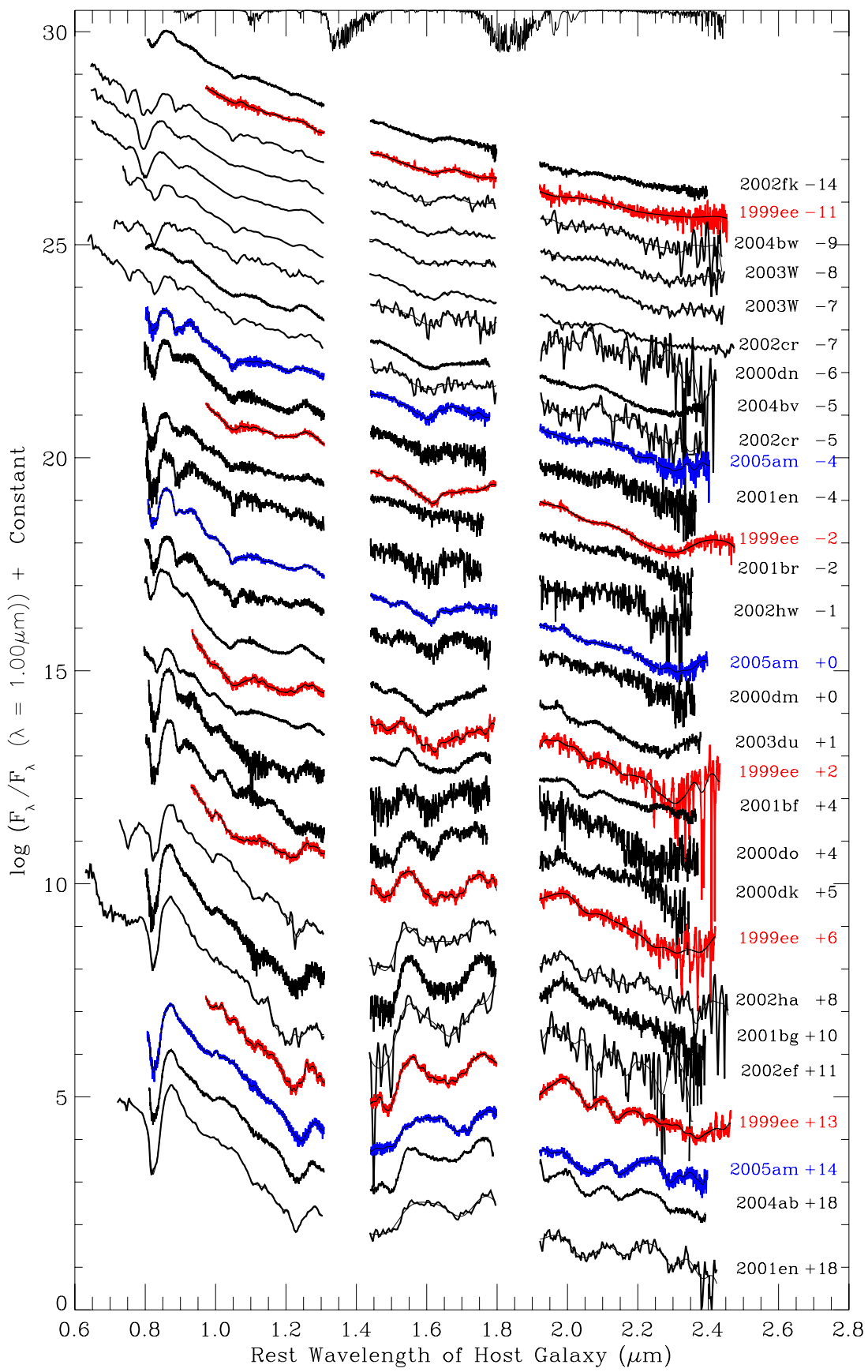

Figure 18. Uniformity of feature evolution in NIR spectra of SNe Ia within a few weeks of maximum light is demonstrated by interleaving "snapshot" data from 19 $\mathrm{SNe}$ Ia in our sample with two time sequences of spectra obtained from individual SNe Ia (see the text in Section 4.1). Spectral shape and feature development with time are found to be consistent in individual events and also between diverse SNe Ia. Five spectra from SN 1999ee obtained between $-11 \mathrm{~d}$ and $+13 \mathrm{~d}$ are shown in red (Hamuy et al. 2002). The four spectra in blue are from our sample and show SN 2005am between $-4 \mathrm{~d}$ and $+14 \mathrm{~d}$.

(A color version of this figure is available in the online journal.)

emitted. For wavelength regions where this line-blanketing occurs, increased flux levels mimic emission features (Wheeler et al. 1998). There are thousands of these lines and they are not individually resolved but they effectively raise the continuum level through the wavelength regions that they occupy by creating a temporary effective photosphere at larger radii. Figure 21 compares model data with four spectra from SN 2005am. The model resolution creates a series of peaks rather than a smooth profile but Doppler shifting will blend the individual lines and the models clearly predict the behavior observed in the data where a pseudophotosphere is created at extended radii that increases the continuum flux in wavelength regions: $0.9-1.2,1.5-1.85$, and $2.05-2.45 \mu \mathrm{m}$.

At 20-30 days after $V_{\max }$, the absorbing material is diluted by expansion to the point where it is no longer possible to define a photosphere. This is a transitional phase between the photospheric epoch and the true nebular phase. In some wavelength regions, line-blanketing from the thousands of $\mathrm{Fe}-$ group lines continues to distort the continuum.

The Ca II IR triplet forms the strongest single feature in each of our spectra. Extremely low excitation values for these lines suggest that they are likely to create absorptions of this 


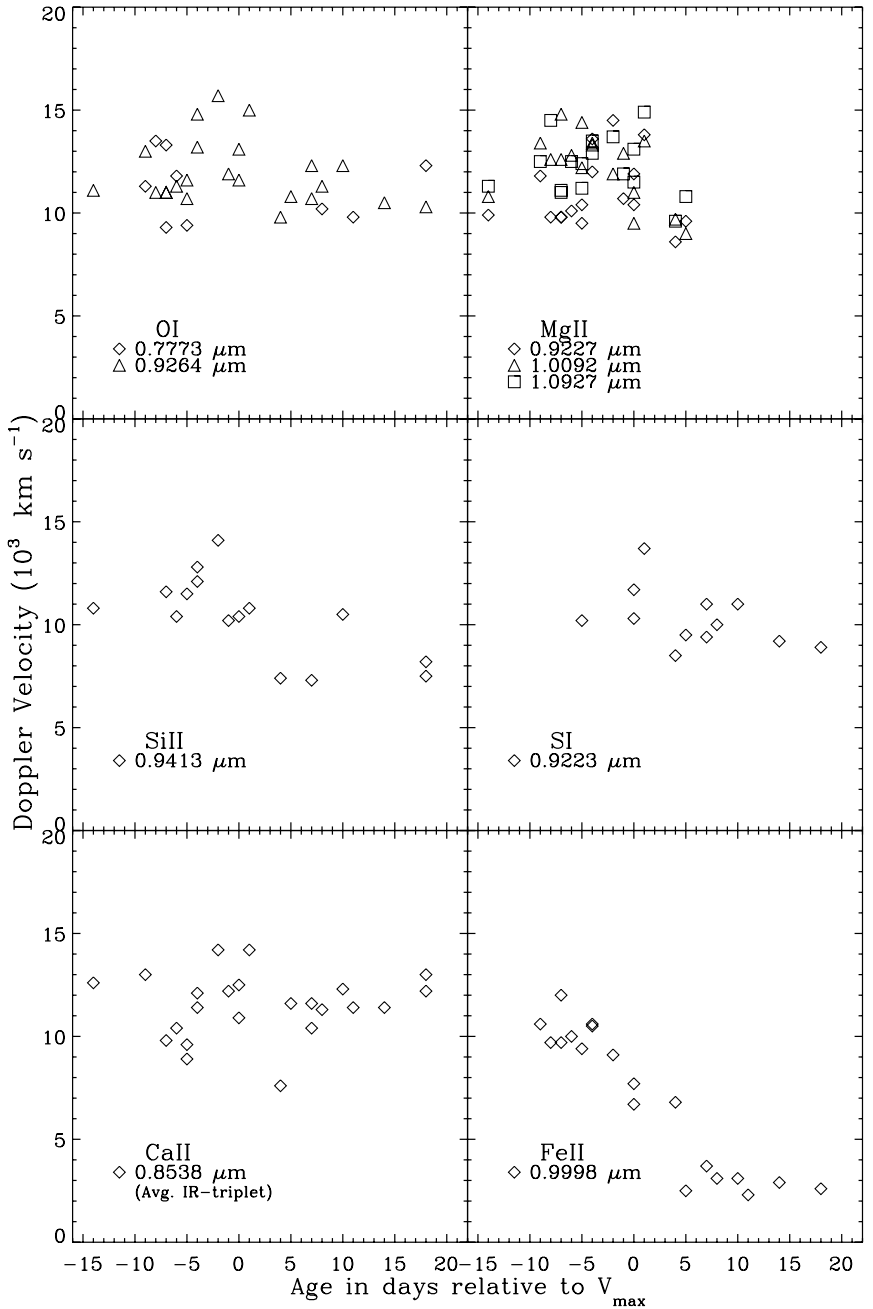

Figure 19. Plot of measured Doppler velocities for ions with confident identifications in spectra obtained before $+20 \mathrm{~d}$. In all panels, the ordinate is the time of observation in days relative to $V_{\max }$, and the abscissa is the Doppler velocity in $10^{3} \mathrm{~km} \mathrm{~s}^{-1}$. The OI data include measurements from two lines $(0.7774$ and $0.9264 \mu \mathrm{m})$, the $\mathrm{Mg}$ II data include three lines $(0.9227,1.0092$, and $1.0927 \mu \mathrm{m})$, and there is one line each from Si II $(0.9413 \mu \mathrm{m}), \mathrm{S}$ I $(0.9223 \mu \mathrm{m})$, the Ca II IR triplet $(0.8538 \mu \mathrm{m}$ blended line $)$, and Fe II $(0.9998 \mu \mathrm{m})$. The measurements are compiled in Table 4 and discussed in Section 4.2.

magnitude at very low Ca II abundances. Since solar values in circumstellar material would provide sufficient calcium to generate these features, it is not expected that the P Cygni profile features found with absorption minima near $0.82 \mu \mathrm{m}$ are produced by freshly synthesized Ca II.

\section{SUMMARY}

We present 41 high-quality NIR spectra from SNe Ia obtained between 2 weeks before and 10 weeks after $V_{\max }$. We show that the FT is an excellent method for smoothing SN Ia data to allow more consistent measurements and better comparisons between spectra without compromising the important features. The procedures we describe for fitting continua and measuring absorption features provide consistency for measurement and analysis of a very large number of spectral features from spectra with a wide range of quality and resolution. We estimate relative line strengths for the strongest lines from ions expected to be found in spectra from $\mathrm{SNe}$ Ia in this wavelength range and provide tables of the estimates as a resource for exploring NIR spectra.

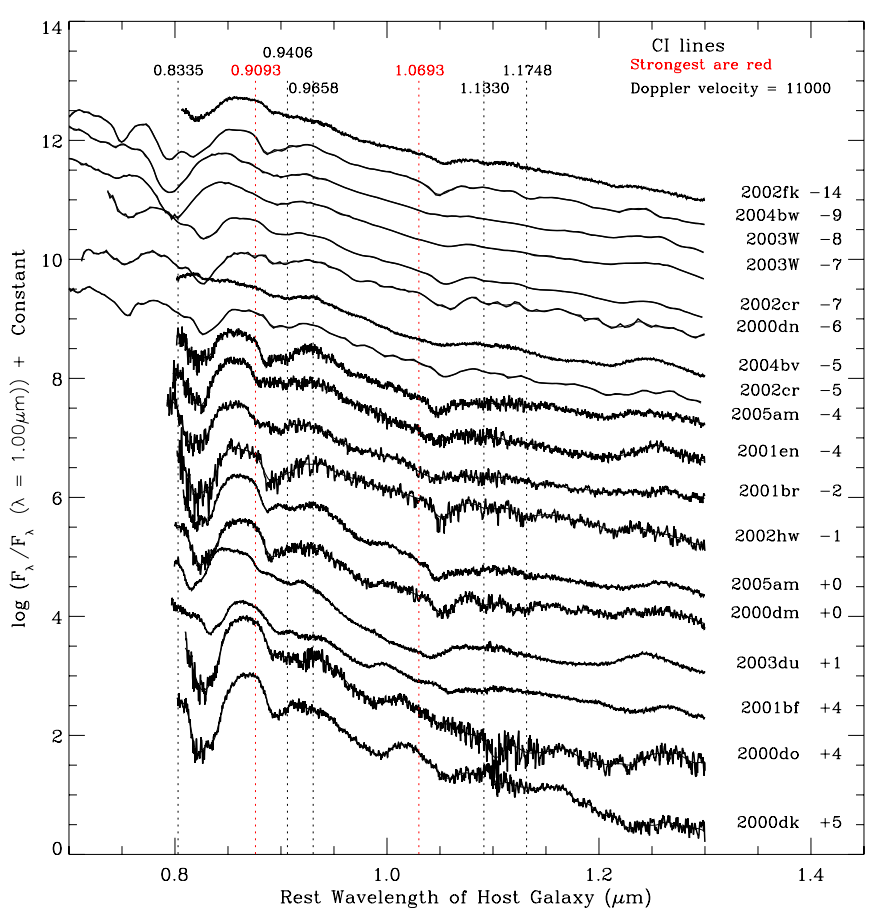

Figure 20. Eighteen NIR spectra from SNe Ia as in Figure $12(-14 \mathrm{~d}$ to $+5 \mathrm{~d})$. The locations of strong $\mathrm{C}_{\mathrm{I}}$ lines marked in the figure at Doppler velocities of $11,000 \mathrm{~km} \mathrm{~s}^{-1}$ which is typical for O I during this epoch. The strongest lines, as indicated in Table 6, are marked in red and the weaker lines appear in black. Small features appear in some spectra near the expected location for lines from $0.9406,1.1330$, and $1.1754 \mu \mathrm{m}$, but if these lines produce absorption features then the stronger lines at 1.0693 and $0.9093 \mu \mathrm{m}$ should be detected and they are not. The possible feature for the $0.9406 \mu \mathrm{m}$ line is more likely attributed to Si II at $0.9413 \mu \mathrm{m}$ (see Section 4.3)

(A color version of this figure is available in the online journal.)

By comparing true time series of spectra with "snapshot" spectra from many individual SNe Ia, we show that the evolution of spectral features is consistent for NIR spectra from normal $\mathrm{SNe}$ Ia. Because geometrical dilution of the $\mathrm{SN}$ reveals deeper layers within the expanding envelope, our sample of 41 spectra obtained between $-14 \mathrm{~d}$ and $+75 \mathrm{~d}$ provides an excellent record of the physical properties of $\mathrm{SNe}$ Ia from the outer layers to deep within the envelope.

The spectra disclose that burning products are distributed in well-defined, radial layers. This is an important result that constrains the progenitor evolution and the burning physics. Table 4 and Figure 19 show the measured Doppler velocities for O I, Mg II, Si II, S I, Ca II, and Fe II. Burning products from the lowest burning densities appear at the highest velocities (O I and $\mathrm{Mg}$ II at $\approx 10,000-15,000 \mathrm{~km} \mathrm{~s}^{-1}$ ) with well-defined minima. High velocities correspond to the outer layers of SNe Ia and clearly defined minimum velocities indicate that the lineforming region containing $\mathrm{O}$ and $\mathrm{Mg}$ has a distinct inner edge. Si II, S I, and Mn II are found at somewhat lower velocities $\left(\approx 8000-12,000 \mathrm{~km} \mathrm{~s}^{-1}\right)$ and they also have clear velocity boundaries to the line-forming region. The agreement in spectral features from a large number of SNe Ia eliminates the possibility that observations of finite line-forming regions are due to clumps of material.

$\mathrm{Ca}$ II is only detected in the IR triplet despite expectations that other Ca II lines would be present. Due to the very low excitation values for these lines, this result implies that detection is not due to freshly synthesized material. Fe II is detected at a wide range of velocities $\left(\approx 2000-12,000 \mathrm{~km} \mathrm{~s}^{-1}\right)$ with no apparent minimum. 


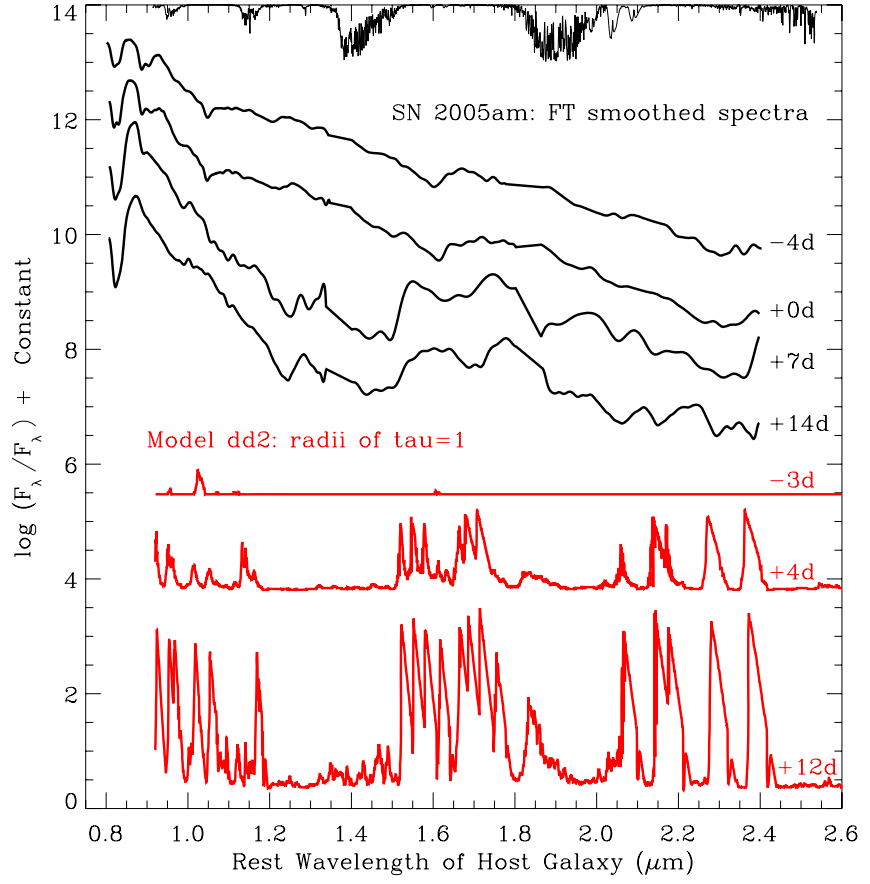

Figure 21. Model data for radius at $\tau=1.0$ as a function of wavelength compared to spectra obtained from SN 2005 am at epochs: $-4 \mathrm{~d},+0 \mathrm{~d},+7 \mathrm{~d}$, and $+14 \mathrm{~d}$. The models clearly predict the observed behavior of a pseudophotosphere created at extended radii that increases the continuum flux in wavelength regions: $0.9-1.2,1.5-1.85$, and $2.05-2.45 \mu \mathrm{m}$. Increased opacity due to lineblanketing from thousands of Fe-group lines increases the effective radius of the photosphere at certain wavelengths. The model resolution creates individual peaks but groups of lines will be blended by Doppler shifting to smear out the sharp features (see the text in Section 5.3).

(A color version of this figure is available in the online journal.)

The distribution of burning products and radially stratified chemical structure is consistent with the explosion occurring in a $\mathrm{C} / \mathrm{O}$ WD with a radial density gradient and lower densities on the outside (Section 5.2). Our results agree with models that predict explosive carbon burning will occur in the extreme outer layers of SNe Ia. Oxygen burning and partial silicon burning appear to form intermediate layers and some Si must be completely burned to produce Fe detected at extended radii. There are small overlaps between adjacent layers, but our data eliminate the possibility of large-scale mixing of lower mass elements to the center or Fe-group elements to the extreme outer layers.

$\mathrm{C}, \mathrm{O}$, and $\mathrm{Mg}$ are only detectable by spectral observations up to a few days after $V_{\max }$, so it is useful that there are 22 spectra in our sample obtained at $+5 \mathrm{~d}$ or earlier. We do not find carbon in the spectra at any epoch but we do find carbon burning products $(\mathrm{O}$ and $\mathrm{Mg})$ occupying the same physical space in the outer layers of the SNe. There are strong lines from $\mathrm{C}_{\mathrm{I}}$ in the NIR that have produced detections in subluminous SNe Ia, but $\mathrm{C}_{\mathrm{I}}$ is not found in our sample. ( $\mathrm{C}_{\mathrm{II}}$ lines are expected to be weaker than $\mathrm{C}_{\mathrm{I}}$ and are also not detected.) $\mathrm{O}_{\mathrm{I}}$ and $\mathrm{Mg} \mathrm{II}$ are both products of explosive carbon burning and they are detected in nearly the same velocity space in the outer layers. The line strengths for $\mathrm{C}$ and $\mathrm{O}$ will be similar for the same ionization stages, so if the $\mathrm{O}_{\mathrm{I}}$ in our spectra is due to unburned material, then $\mathrm{C}$ I should also be present in detectable amounts but there is no evidence for C $\mathrm{I}$ (Marion et al. 2006).

These results strongly suggest that the entire progenitor is consumed in normal SNe Ia which implies that nearly the same amount of fuel is used to power each explosion and helps to explain the uniformity in $\mathrm{SNe}$ Ia observations. These results constrain burning physics and models on a physical rather than phenomenological basis.

The size of this sample also facilitates the search for secondary relationships with other data libraries that may further constrain SN Ia physics and improve the effectiveness of SNe Ia as cosmological distance indicators. Well-sampled light curves for the SNe in our sample will allow comparison of well-studied photometric parameters with NIR line strengths. NIR observables can now be compared to empirical patterns such as the relationship between peak brightness and light curve shape, $B-V$ color differences, secondary peaks in the IR, and host galaxy morphology. This sample may enable accurate " $K$ corrections" for photometric observations in the $J, H$, and $K$ bands. NIR spectra may also provide insights into areas of $\mathrm{SNe}$ Ia physics such as progenitor ZAMS mass, metallicity, rotation, asymmetry in the explosion, and gravitational influence on the detonation (Höflich et al. 1998, 2002; Wang et al. 1997; Yoon \& Langer 2004a, 2004b). We hope that this sample will truly open the door to the use of NIR data for productive SNe Ia research.

G.H.M. is a Visiting Astronomer at the IRTF, which is operated by the University of Hawaii under Cooperative Agreement NCC 5-538 with the National Aeronautics and Space Administration, Science Mission Directorate, Planetary Astronomy Program. We want to express our appreciation to the individuals at the IRTF for guidance and help with the observations. In particular, Alan Tokunaga, John Rayner, Mike Cushing, Bill Golisch, Dave Griep, and Paul Sears have been most helpful. We also thank the TAC of the IRTF for support and instructive comments. G.H.M. acknowledges and thank Mike Cushing for helpful comments.

We thank R. Rudy of The Aerospace Corporation and R. Puetter of UCSD for providing observations of SNe 1998aq and 1998bu.

We are grateful to W. D. Li and M. Papenkova for providing light curve data for some of the SNe in our sample, and we thank J. Vinko for his work compiling light curve data.

P.A.H. is supported in part by NSF grants 0703902 and 0708855 .

We thank the referee for constructive comments that have improved the structure and presentation of the paper.

\section{APPENDIX}

\section{DISCUSSION OF INDIVIDUAL FEATURES AND LINE IDENTIFICATIONS}

This appendix discusses line identifications for specific features in the spectra. Line identifications and measured Doppler velocities are provided where possible. Individual lines are referred to by their rest wavelengths. The spectra are grouped by epoch using the same groups as in Section 4.

Velocity measurements discussed in this appendix can be found in Table 4 and Figure 19.

\section{A.1. A Very Early Spectrum (14 days Before $\left.V_{\max }\right)$}

Discussion in this appendix makes reference to Figure 11, which shows the spectrum from SN 2002fk which was obtained 14 days before $V_{\max }$. Features are labeled in the figure for which we have confident identifications.

He I. He I is not detected in this spectrum from either of the strongest $\mathrm{He}$ I lines in the NIR found at 1.0830 or $2.0581 \mu \mathrm{m}$. 
Possible associations for He I include the absorption feature found near $1.05 \mu \mathrm{m}$ which corresponds to a Doppler velocity of $8600 \mathrm{~km} \mathrm{~s}^{-1}$ for the $1.0830 \mu \mathrm{m}$ line. This is an unrealistically low velocity because if $\mathrm{He} \mathrm{I}$ is present, it is expected to be found only in the extreme outer layers of the $\mathrm{SN}$ at high velocities. The $1.05 \mu \mathrm{m}$ feature has a plausible alternative identification as $\mathrm{Mg}$ II from the $1.0927 \mu \mathrm{m}$ line and it is labeled as such in the figure. The $2.0581 \mu \mathrm{m} \mathrm{He}$ I line (which is expected to be 6-12 times weaker than the $1.083 \mu \mathrm{m}$ line depending on temperature), can possibly be associated with either of the two weak features at 2.00 and $2.04 \mu \mathrm{m}$ with velocities of 9700 or $2500 \mathrm{~km} \mathrm{~s}^{-1}$ which are inconsistent with $8600 \mathrm{~km} \mathrm{~s}^{-1}$ if the strongest He I line at $1.0830 \mu \mathrm{m}$ produces the $1.05 \mu \mathrm{m}$ feature.

C I. $C_{\text {I }}$ is predicted to generate strong lines at 1.0693, 0.9093, 0.9406 , and $1.1754 \mu \mathrm{m}$ in order of expected strength (see Table 5). Additional discussion of $\mathrm{C}_{\mathrm{I}}$ is found in Section 4.3 and illustrated in Figure 20. Very weak features can be seen in this spectrum by optimistic observers at Doppler velocities of 10,000-12,000 $\mathrm{km} \mathrm{s}^{-1}$, but no confident detections can be made and the velocities are not consistent within each spectrum. Some models for $\mathrm{SNe}$ Ia predict that $\mathrm{C}_{\mathrm{I}}$ will be present in the extreme outer layers, but we are unable to identify $\mathrm{C}_{\mathrm{I}}$ in this, or any other spectrum in our sample.

$\mathrm{O}$ I. $\mathrm{O}_{\mathrm{I}}$ is likely from the $0.9264 \mu \mathrm{m}$ line contributing to the absorption feature found near $0.89 \mu \mathrm{m}$ at $11,500 \mathrm{~km} \mathrm{~s}^{-1}$ in a blend with $\mathrm{Mg}$ II $(0.9227 \mu \mathrm{m})$. The 0.8446 O I line may also contribute to the absorption from the Ca II IR triplet near 0.81 but this wavelength is at the extreme edge of the spectrum and it is difficult to evaluate the presence of small features in this region due to the strength of the Ca II feature. The O I $0.7773 \mu \mathrm{m}$ line is expected to be 3-20 times stronger than the 0.9264 line and the $0.7773 \mu \mathrm{m}$ line is detected in all early spectra from which we have coverage of the wavelength region down to $0.7 \mu \mathrm{m}$ but this spectrum does not cover that range.

Mg II. Mg II produces strong features that are clearly detected in this spectrum (and labeled in Figure 11) from $0.9227 \mu \mathrm{m}$, found near $0.89 \mu \mathrm{m}$ at $10,300 \mathrm{~km} \mathrm{~s}^{-1}$, and $1.0927 \mu \mathrm{m}$, found near $1.05 \mu \mathrm{m}$ at $11,300 \mathrm{~km} \mathrm{~s}^{-1}$. The measured velocity for the $0.9227 \mu \mathrm{m}$ line is $1000 \mathrm{~km} \mathrm{~s}^{-1}$ less than the measured velocity for the $1.0927 \mu \mathrm{m}$ line due to intrusion on the blue side of the $\mathrm{Mg}$ II absorption from the emission component of the very strong $\mathrm{Ca}$ II IR triplet. This line is also likely to be blended with $\mathrm{O}$ I at $0.9264 \mu \mathrm{m}$. The high quality of this spectrum reveals a probable detection near $0.97 \mu \mathrm{m}$ for the $\mathrm{Mg}$ II line at $1.0092 \mu \mathrm{m}$ with a Doppler velocity of $10,900 \mathrm{~km} \mathrm{~s}^{-1}$. This line is predicted to be 5-20 times weaker than the $0.9227 \mu \mathrm{m}$ line which is consistent with the observed feature strengths. The absorption feature near $1.60 \mu \mathrm{m}$ receives contributions from $\mathrm{Mg}$ II at $1.6787 \mu \mathrm{m}$ with $11,600 \mathrm{~km} \mathrm{~s}^{-1}$ in addition to Si II $(1.6930 \mu \mathrm{m})$ and Co II (many lines $1.57-1.77 \mu \mathrm{m}$ ). Weak evidence can be found for $\mathrm{Mg}$ II from the 0.9632 and $2.1369 \mu \mathrm{m}$ lines at similar velocities.

Si II. Si II from the strong line at $0.9413 \mu \mathrm{m}$ is the likely source of the feature found near $0.91 \mu \mathrm{m}$ at a Doppler velocity of $11,300 \mathrm{~km} \mathrm{~s}^{-1}$. This velocity is similar to the velocities measured for $\mathrm{Mg}$ II which is consistent with models for this epoch. The 1.6930 Si II line may be part of the $1.6 \mu \mathrm{m}$ absorption feature but the velocity to the minimum of the feature is nearly $18,000 \mathrm{~km} \mathrm{~s}^{-1}$ for this line. This ion may also contribute to the feature found near $1.09 \mu \mathrm{m}$ from the $1.1311 \mu \mathrm{m}$ line in a blend with Si III and Fe III. However, Si II at $1.1311 \mu \mathrm{m}$ is a much weaker line than the $0.9413 \mu \mathrm{m}$ line, and the velocity does not correlate with other Si II lines. If there is sufficient $\mathrm{Si}$ II to produce a feature of this magnitude, then detections should also be possible from lines at 1.1737 and $1.7183 \mu \mathrm{m}$. There is no evidence in this spectrum for these other Si II lines.

Si III. Si III can be present in the same spectrum with Si II if the photosphere is deep within the envelope so that temperature and pressure differences between absorbing regions near the photosphere and in the outer layers contain different ionization stages of the same element (see Section 5.3). That scenario suggests that Si III will be found in slower and hotter lineforming regions near to the photosphere. Due to a relatively low excitation minimum and high oscillator strength, the Si III $0.9324 \mu \mathrm{m}$ line is predicted to be $\sim 40$ times stronger at $10,000 \mathrm{~K}$ than the next strongest Si III line in the NIR. This line may contribute to the feature observed near $0.91 \mu \mathrm{m}$ at a Doppler velocity of $8400 \mathrm{~km} \mathrm{~s}^{-1}$ in a blend with Si II and $\mathrm{Mg}$ II. A table of estimated line strengths using a temperature of 10,000 K shows change in the relative line strengths for Si III compared to $5000 \mathrm{~K}$ (see Table 6). For example, the fact that the Si III $1.0526 \mu \mathrm{m}$ line is not detected in this spectrum is consistent with higher temperatures in the Si III line-forming region. Other possible identifications for $\mathrm{Si}$ III are the feature at $1.22 \mu \mathrm{m}$ from the $1.2541 \mu \mathrm{m}$ line (a blend of 1.2523 and $1.2601 \mu \mathrm{m}$ ) at $9600 \mathrm{~km} \mathrm{~s}^{-1}$ and $1.09 \mu \mathrm{m}$ from $1.1341 \mu \mathrm{m}$ at10,500 $\mathrm{km} \mathrm{s}^{-1}$.

Ca II IR triplet. The Ca II IR triplet is strong in this spectrum from SN 2002fk with a Doppler velocity of $12,300 \mathrm{~km} \mathrm{~s}^{-1}$. The velocity measurement is based on a blend of the 0.8498 and $0.8542 \mu \mathrm{m} \mathrm{Ca}$ II lines, giving a mean wavelength of $0.8538 \mu \mathrm{m}$, and measured with respect to the bluer and deeper component of the absorption feature. Resolution is sufficient to separate this pair from the other member of the triplet found at $0.8662 \mu \mathrm{m}$. The shape of the absorption feature is consistent with estimates that the 0.8538 blend is about twice as strong as the 0.8662 line.

The very strong P Cygni profile from the Ca II IR triplet may not be produced by freshly synthesized $\mathrm{Ca}$ (Section 5.3). There is no evidence in the spectrum for $\mathrm{Ca}$ II from the next strongest lines at 1.1876 or $0.9906 \mu \mathrm{m}$ and this may indicate that Ca II produced in the explosion is not detectable.

This SXD spectrum lacks coverage in the wavelength region to detect a possible high-velocity component from $\mathrm{Ca}$ II.

Fe II. Fe II lines are more difficult to compare based on our simple line-strength estimates because the actual line strengths are affected by multiple transitions. As a consequence, the simple calculations in our tables for the relative estimated strength of each Fe II line may be less accurate than the estimates for smaller atoms. The Fe II $0.9998 \mu \mathrm{m}$ line is a possible source for the feature found near $0.97 \mu \mathrm{m}$ in this spectrum, but the velocity would be only $8100 \mathrm{~km} \mathrm{~s}^{-1}$. This velocity is similar to some of the conjectured Si III velocities. The $1.0500 \mu \mathrm{m}$ line from Fe II is not detected, but the 1.0863 line may be a part of the feature near $1.05 \mu \mathrm{m}$ in a blend with $\mathrm{Mg}$ II. The $1.1126 \mu \mathrm{m}$ Fe II line is a possible contributor near $1.09 \mu \mathrm{m}$ in a blend with Si III.

Fe III. Fe III at 0.9124 is possible in a blend with $\mathrm{O}_{\mathrm{I}}$ and $\mathrm{Mg}$ II but the Doppler velocity at the absorption minimum is very low at $7100 \mathrm{~km} \mathrm{~s}^{-1}$. As noted in the discussion for $\mathrm{Mg} \mathrm{II}$ in this appendix, this absorption feature is distorted by emission from the Ca II triplet and the true velocity may be higher. This ion has several lines from 1.60 to $1.67 \mu \mathrm{m}$ that may add to the $1.6 \mu \mathrm{m}$ feature with the strongest line at $1.6697 \mu \mathrm{m}$ having a velocity of $9900 \mathrm{~km} \mathrm{~s}^{-1}$ at the minimum. The $1.1323 \mu \mathrm{m}$ line may also 
form, or contribute to, the absorption near $1.09 \mu \mathrm{m}$ at a velocity of $10,000 \mathrm{~km} \mathrm{~s}^{-1}$.

Co II. Co II is detected at $\sim 12,000 \mathrm{~km} \mathrm{~s}^{-1}$ from individual lines at $1.7772,2.2205,2.4596$, and $2.3613 \mu \mathrm{m}$. The $1.7462 \mu \mathrm{m}$ line is not found in this spectrum. Numerous Co II lines between 1.57 and $1.77 \mu \mathrm{m}$ are likely contributors to the $1.60 \mu \mathrm{m}$ feature.

\section{A.2. The Photospheric Phase (Outer Layers; $-14 d$ to $+5 d$ with Respect to $V_{\text {max }}$ )}

During this epoch, the photosphere is expected to be in the outer layers of the $\mathrm{SN}$ and the line-forming regions will be close to the photosphere. Discussion in this appendix makes reference to Figure 12 showing 18 spectra obtained between -14 and +5 days with respect to $V_{\max }$ and also to Figure 13 that displays four representative spectra from this group: SNe 2004bw (LRS obtained at -9d), 2005am (SXD obtained at -4d), 2000dm (SXD obtained at +0d), and 2001bf (SXD obtained at $+4 d$ ) with features labeled for which we have confident identifications. These figures only display the spectra in the wavelength region $0.65-1.35 \mu \mathrm{m}$ to permit detailed inspection of the region where most of the features occur in this epoch. The complete spectra from 0.7 to $2.5 \mu \mathrm{m}$ can be viewed in Figure 10.

C I. C I is not detected from any of these spectra. The strongest $\mathrm{C}$ I lines are found at 1.0693, 0.9087, 0.9406, and $0.9639 \mu \mathrm{m}$, listed in order of expected strength (see Figure 20 and Section 4.3 for additional discussion of $\mathrm{C}_{\mathrm{I}}$ in our sample).

O I. O I at 0.7773 forms the feature near $0.75 \mu \mathrm{m}$ detected in all LRS spectra in this group (the SXD spectra do not cover the wavelength region of this line). Table 4 shows that the Doppler velocity of this line is measured at $11,300-13,500 \mathrm{~km} \mathrm{~s}^{-1}$ except for velocities of 9300 and 9400 measured in the two spectra from SN 2002cr. (Note that the spectra from SN 2002cr exhibit lower velocities than other spectra for all explosion products.) The O I feature is strong in all spectra from this group that include the appropriate wavelength region.

The $0.9263 \mu \mathrm{m}$ line from $\mathrm{O}_{\mathrm{I}}$ is expected to be $3-17$ times weaker than the $0.7773 \mu \mathrm{m}$ line but it is likely to contribute to the $0.89 \mu \mathrm{m}$ feature in a blend with $\mathrm{Mg}$ II $0.9227 \mu \mathrm{m}$ at velocities of 11,000-15,000. Possible evidence for the $1.1287 \mu \mathrm{m}$ O I line (which should be half as strong as the $0.9263 \mu \mathrm{m}$ line) appears near $1.09 \mu \mathrm{m}$ in some of these spectra.

The strongest features at $1.09 \mu \mathrm{m}$ occur in spectra from SNe 2002hw (-1d) and 2000dm (+1d) and correlate with the strongest absorptions at $0.89 \mu \mathrm{m}$ which is suggestive that $\mathrm{O}_{\mathrm{I}}$ contributes to both features.

Mg II. Mg II is found in this group producing strong features from lines at 0.9227 and $1.0927 \mu \mathrm{m}$ and weaker but detectable features in higher $\mathrm{S} / \mathrm{N}$ spectra from lines at 1.0092 and $1.6787 \mu \mathrm{m}$. The Doppler velocities for the 1.0927 line are $11,100-14,500 \mathrm{~km} \mathrm{~s}^{-1}$, while the measured velocities for the 0.9227 line are $\sim 1000 \mathrm{~km} \mathrm{~s}^{-1}$ slower as a result of the enormous P Cygni emission from $\mathrm{Ca}$ II that impinges on the blue side of this absorption feature (Table 4). Stronger Mg II features are often correlated with higher measured Doppler velocities. As they were for $\mathrm{OI}$, the lowest velocities are found in the spectrum of SN 2002cr. Figures 9 and 12 show that there is an obvious correlation between strong spectral features from $\mathrm{Mg}$ II at $1.0927 \mu \mathrm{m}$ and the strongest features from the $0.9227 \mu \mathrm{m}$ line. The final spectrum (in temporal order) to show evidence of $\mathrm{Mg}$ II (SN 2000dk, $+5 \mathrm{~d})$ clearly shows features from both of these strong lines.

The $\mathrm{Mg}$ II line at $0.7890 \mu \mathrm{m}$ may contribute to the $0.75 \mu \mathrm{m}$ feature in a blend with $\mathrm{O}$ I but the velocities to the absorption minima of this feature would be $14,000-18,000 \mathrm{~km} \mathrm{~s}^{-1}$ and that is inconsistent with other $\mathrm{Mg}$ II measurements. The $0.7890 \mu \mathrm{m}$ line may flatten the P Cygni emission component of the $\mathrm{O}$ i line as in the spectrum from SN 2002cr.

The $1.0092 \mu \mathrm{m} \mathrm{Mg}$ II line is expected to be 2 orders of magnitude weaker than $1.0927 \mu \mathrm{m}$ but it is detectable in most spectra in this group, forming a weak absorption near $0.97 \mu \mathrm{m}$ at velocities comparable to the other $\mathrm{Mg}$ II lines. This feature is detected in all but the noisiest spectra from this group and it often exhibits a double or triple bottom containing minima near $0.95,0.97$, and $0.99 \mu \mathrm{m}$ with $0.97 \mu \mathrm{m}$ being the strongest. The 0.95 and $0.99 \mu \mathrm{m}$ features are smaller than the $1 \sigma$ noise but are consistent from spectrum to spectrum in magnitude and location relative to the central feature. The central feature is probably $\mathrm{Mg}$ II at $1.0092 \mu \mathrm{m}$, while candidates for the others are Ca II at $0.9906 \mu \mathrm{m}$ and Fe II at $0.9998 \mu \mathrm{m}$. The fact that the feature near $0.97 \mu \mathrm{m}$ becomes stronger after maximum light suggests that the primary source of absorption at this wavelength may be shifting from $\mathrm{Mg}$ II to Fe II.

Velocity measurements of the $\mathrm{Mg}$ II line at $1.6787 \mu \mathrm{m}$ and the $1.60 \mu \mathrm{m}$ absorption feature correspond closely to the velocities of the other $\mathrm{Mg}$ II lines. This broad feature probably receives contributions from other ions, notably Si II and Co II. The absorption feature is particularly strong in spectra that exhibit other strong $\mathrm{Mg}$ II features, such as: SN 2005am (at $-2 \mathrm{~d}$ and $+2 \mathrm{~d})$, SNe $2002 \mathrm{hw}(-1 \mathrm{~d})$, and 2000dm (+1d). That may indicate increased abundance of $\mathrm{Mg}$ II near max light. Mg II lines at 0.9632 and $2.1369 \mu \mathrm{m}$ are also possible in weak features or blends.

Inspection of the complete sample (Figure 9) shows that all 22 spectra in our sample obtained at or before $+2 \mathrm{~d}$ show strong $\mathrm{Mg}$ II features near 0.89 and $1.05 \mu \mathrm{m}$. Even spectroscopically unusual SNe Ia such as 2004bv exhibit distinct Mg II signatures. Measured velocities for $\mathrm{Mg}$ II are given in Table 4 and plotted in Figure 19.

Si II. Si II from the $0.9413 \mu \mathrm{m}$ line is the probable source of the feature found near $0.91 \mu \mathrm{m}$. Note that this feature is distinct from the absorption found near $0.89 \mu \mathrm{m}$ and it does not appear in all spectra. The measured velocities are between 10,200 and $14,100 \mathrm{~km} \mathrm{~s}^{-1}$. As noted in the discussion of the $\mathrm{Mg}$ II line at $0.9227 \mu \mathrm{m}$, impingement from the emission component of the Ca II triplet may affect the location of this feature, pushing it toward the red. The $0.91 \mu \mathrm{m}$ feature, and thus the $0.9413 \mu \mathrm{m}$ Si II line, is not detected in spectra from SNe 2004bw (-9d), 2003W (-7d and $-6 \mathrm{~d}), 2002 \mathrm{hw}(-1 \mathrm{~d}), 2000 \mathrm{dm}(+0 \mathrm{~d})$, or 2000dk (+5d). In general, the spectra without this Si II detection show very strong Mg II features that may obscure the Si II signal due to P Cygni emission from Mg II at $0.9227 \mu \mathrm{m}$.

The $1.6930 \mu \mathrm{m}$ line from Si II is likely to contribute to the $1.60 \mu \mathrm{m}$ feature but velocities to the absorption minima are $14,000-18,000 \mathrm{~km} \mathrm{~s}^{-1}$ which is higher than other measured Si II velocities including measurements during this epoch of the strong Si II features at optical wavelengths. The line at $0.7846 \mu \mathrm{m}$ may contribute to the $0.75 \mu \mathrm{m}$ feature in a blend with O I but velocities to the absorption minima are 12,200$16,400 \mathrm{~km} \mathrm{~s}^{-1}$ which is about $2000 \mathrm{~km} \mathrm{~s}^{-1}$ higher than for the $0.9413 \mu \mathrm{m}$ line. Possible evidence for the $1.1748 \mu \mathrm{m}$ line appears near $1.13 \mu \mathrm{m}$ in four of the spectra at plausible 
velocities of 10,100-11,900. However, three of the four spectra with $1.13 \mu \mathrm{m}$ features are the ones that do not have a $0.91 \mu \mathrm{m}$ feature, making the Si II association unlikely.

Si III. Si III is not detected in this group except for the earliest spectrum at $-14 \mathrm{~d}$ and discussed in the previous appendix.

S I. S I probably begins to contribute to the $0.89 \mu \mathrm{m}$ feature after maximum light from $0.9223 \mu \mathrm{m}$ line at velocities of 10,000 $12,000 \mathrm{~km} \mathrm{~s}^{-1}$. But the S I $1.0457 \mu \mathrm{m}$ line, which is only three times weaker than the $0.9223 \mu \mathrm{m}$ line, is not detected.

S II. S II is not detected in these data but all of the strongest lines for this ion are on the blue side of $0.83 \mu \mathrm{m}$, so their Doppler shifted positions will not be included in the SXD spectra that comprise the majority of spectra (and highest resolution) in this group.

Ca II IR triplet. The Ca II IR triplet dominates the spectra in this group, forming the $0.82 \mu \mathrm{m}$ feature with velocities near $12,000 \mathrm{~km} \mathrm{~s}^{-1}$ for most SNe in our sample. The only exception is the spectrum from SN 2004bv (-4d) which may be an SN 1991T-like object in which the Ca II is absent from early spectra but present, as in spectra from normal $\mathrm{SNe} I a$, at later times. The spectrum in this sample from SN 2004bv at +60d shows the $\mathrm{Ca}$ II and seems to be normal.

The square bottom of the $\mathrm{Ca}$ II absorption region in these features is due to separately resolved contributions from two parts of the triplet. On the blue side of the absorption feature, we use a mean wavelength of $0.8538 \mu \mathrm{m}$ for the blend, weighted by oscillator strength, for the pair of lines at 0.8498 and $0.8542 \mu \mathrm{m}$. The $0.8662 \mu \mathrm{m}$ line can be easily identified in these spectra as a separate part of the feature at about one half the predicted strength for the $0.8538 \mu \mathrm{m}$ line. To measure Doppler velocities, we use the location of the minimum at the blue side of the feature and compare it to the $0.8538 \mu \mathrm{m}$ rest wavelength of the blend.

The shape of the emission region forming the $\mathrm{P}$ Cygni profile apparently reflects the shape of the absorption region. Flat or round shapes in the absorption component correspond to similar but inverted profiles in the emission component.

High-Velocity Ca II. The spectrum of SN 2004bw (-9d) shows two distinct minima for Ca II with one at $13,400 \mathrm{~km} \mathrm{~s}^{-1}$ and a high-velocity component at $24,000 \mathrm{~km} \mathrm{~s}^{-1}$. Both spectra from SN 2003W ( $-7 \mathrm{~d}$ and $-6 \mathrm{~d}$ ) have Ca II velocities above $22,100 \mathrm{~km} \mathrm{~s}^{-1}$ without a lower velocity component. The spectra from SN 2002cr ( $-7 \mathrm{~d}$ and $-5 \mathrm{~d})$ appear to have a fading high-velocity Ca II component at $18,800 \mathrm{~km} \mathrm{~s}^{-1}$ and a lower velocity Ca II absorption at $9700 \mathrm{~km} \mathrm{~s}^{-1}$. (Both spectra from SN 2002cr exhibit lower velocities for all measured features than other spectra at similar epochs.) SN 2000dn (-6d) also has a weaker Ca II feature near $22,000 \mathrm{~km} \mathrm{~s}^{-1}$ and a strong Ca II absorption near $10,400 \mathrm{~km} \mathrm{~s}^{-1}$. All LRS spectra obtained at $-5 \mathrm{~d}$ or earlier appear to have high-velocity $\mathrm{Ca}$ II components with velocities measured between 18,800 and $24,000 \mathrm{~km} \mathrm{~s}^{-1}$. Since the high-velocity features have rounded bottoms, we determine the Doppler velocities for the high-velocity Ca II feature using $0.8579 \mu \mathrm{m}$ as the mean wavelength for a blend of all three strong lines in the Ca II IR triplet.

Other Ca II. Other Ca II may be detected in some spectra near $1.14 \mu \mathrm{m}$ from the $1.1876 \mu \mathrm{m}$ line at velocities of 12,000 $16,300 \mathrm{~km} \mathrm{~s}^{-1}$. The spectra in which this feature is the most obvious also have the flattest tops to the IR-triplet emission and that could be due to the next strongest Ca II line at $0.8921 \mu \mathrm{m}$ at similar velocities. The feature attributed to $\mathrm{Mg}$ II near $0.97 \mu \mathrm{m}$ may have a contribution from Ca II at $0.9906 \mu \mathrm{m}$ but the velocity would be $\sim 8000 \mathrm{~km} \mathrm{~s}^{-1}$ which is low. The weak absorption feature near $0.97 \mu \mathrm{m}$ often exhibits a double or triple bottom with minima near $0.95,0.97$, and $0.99 \mu \mathrm{m}$ with 0.97 being the strongest. The 0.95 and $0.99 \mu \mathrm{m}$ features are smaller than the $1 \sigma$ noise but are present in most spectra. An absorption near $0.95 \mu \mathrm{m}$ created by Ca II at $0.9906 \mu \mathrm{m}$ would have a Doppler velocity of $12,300 \mathrm{~km} \mathrm{~s}^{-1}$.

Mn II. Mn II produces no unambiguous detections but the Mn II lines at 0.7414 and $0.7342 \mu \mathrm{m}$ are a possible source for the feature found near $0.70 \mu \mathrm{m}$ in LRS spectra. This identification would require velocities of 14,000-17,000 $\mathrm{km} \mathrm{s}^{-1}$ which are higher than those found for most of the other species. The strong Mn II line at $0.9406 \mu \mathrm{m}$ may contribute to the feature near $0.91 \mu \mathrm{m}$ either alone or in a blend with Si II at velocities that are $\sim 300 \mathrm{~km} \mathrm{~s}^{-1}$ less than values given for Si II at 0.9413 in Table 4. The 0.8410 line is also possible in the blend dominated by the Ca II IR triplet.

Steep Increase in Flux Found Near $1.55 \mu \mathrm{m}$. The steep increase in flux found near $1.55 \mu \mathrm{m}$ is used by some models to define the transition from partial to complete silicon burning. This feature is suggested in the spectra from this group as early as $+0 \mathrm{~d}$ but it becomes distinctive by $+4 \mathrm{~d}$.

Fe II. Fe II at $0.9998 \mu \mathrm{m}$ probably contributes to the $0.97 \mu \mathrm{m}$ absorption that we have associated with Mg II $(1.0092 \mu \mathrm{m})$. The fact that this feature becomes stronger after max light suggests that the primary source for this absorption is shifting from $\mathrm{Mg}$ II to Fe II. Doppler velocities for the Fe II association with this feature begin above 10,000 at $-4 \mathrm{~d}$ and follows as the photosphere recedes into regions with lower expansion velocities to about $7000 \mathrm{~km} \mathrm{~s}^{-1}+2 \mathrm{~d}$.

The velocities required to match the $0.7712 \mu \mathrm{m} \mathrm{Fe}$ II line with the $0.75 \mu \mathrm{m}$ absorption feature in these spectra are inconsistent with Fe II velocities measured for the $0.9998 \mu \mathrm{m}$ line and with other species found in this group. There is no evidence of Fe II from lines at 0.7462 or $1.0500 \mu \mathrm{m}$. The $1.0863 \mu \mathrm{m}$ line may contribute to the $1.05 \mu \mathrm{m}$ feature in a blend with $\mathrm{Mg}$ II but this line is expected to be $\sim 20$ times weaker than some of the other Fe II lines that are not detected.

Fe-group lines are complicated by many potential transitions at the same wavelength and nearly the same strength within each multiplet so the simple formula we have been using to estimate line strength may not be accurate. In this group, the $0.9998 \mu \mathrm{m}$ line is the only Fe II line detected, although the calculated line strengths suggest that lines at $0.7462,0.7712$, and $1.0500 \mu \mathrm{m}$ should also be detected.

Co II. Co II probably contributes to the $1.60 \mu \mathrm{m}$ feature with numerous Co II lines between 1.57 and $1.77 \mu \mathrm{m}$. In a few spectra $(\mathrm{SNe} 2002 \mathrm{hw}(-1 \mathrm{~d}), 2000 \mathrm{dm}(+1 \mathrm{~d})$, and 2005am $(+2 \mathrm{~d}))$, the strongest Co II line $(1.7772 \mu \mathrm{m})$ may be separately resolved at about $11,000 \mathrm{~km} \mathrm{~s}^{-1}$.

Fe-group Blends. Fe-group blends begin to affect the spectra in this group as line-blanketing from thousands of Fe-Group lines results in pseudo-emission features found near $1.52 \mu \mathrm{m}$ and between 1.6 and $2.1 \mu \mathrm{m}$. Model predictions for this effect are compared to the multiple spectra obtained from SN 2005am in Figure 21 and discussed in Section 5.3. Broad humps, that we attribute to unresolved $\mathrm{Fe}$-group lines raising the continuum, are also observed in the spectra from 1.6 to $2.1 \mu \mathrm{m}$ and from 2.1 
to $2.3 \mu \mathrm{m}$. The bump near $1.25 \mu \mathrm{m}$ is very strong early in this group (possibly Si III), weaker through the middle and strong again in the later spectra. These features appear in all spectra from this group.

\section{A.3. The Extended Photospheric Phase (Intermediate Layers; $+4 d$ to $+24 d$ with Respect to $V_{\max }$ )}

During the phase represented by this group, the shape of the continuum becomes influenced by line-blanketing from $\mathrm{Fe}$ group lines which extends the effective photospheric radius (see Section 5.3). Discussion in this appendix refers to Figure 14 showing 10 spectra from $\mathrm{SNe}$ Ia obtained between $+4 \mathrm{~d}$ and $+24 \mathrm{~d}$ with respect to $V_{\max }$ and also to Figure 15 that displays four representative spectra from SNe 2000dk $(+5 \mathrm{~d}), 2001 \mathrm{bg}$ $(+10 \mathrm{~d}), 2004 \mathrm{ab}(+18 \mathrm{~d})$, and 2004da (+24d) with the features labeled for which we have confident identifications.

OI. OI produces the strong absorption near $0.75 \mu \mathrm{m}$ in the LRS spectrum of SN 2002ha (+8d) and this line may also be responsible for the small features at this wavelength up to $+18 \mathrm{~d}$. The $0.9263 \mu \mathrm{m}$ line from $\mathrm{O}$ I is expected to be 3-17 times weaker than the $0.7773 \mu \mathrm{m}$ line but it is likely to be a part of the $0.89 \mu \mathrm{m}$ feature in a blend with $\mathrm{S}$ I at least early in the epoch represented by this group. The measured Doppler velocities for the strongest detections of OI are all $9800 \mathrm{~km} \mathrm{~s}^{-1}$ or higher.

Alternative explanations for the $0.75 \mu \mathrm{m}$ feature are Fe II or Mn II, but the Doppler velocities for these lines do not fit velocities measured for other possible lines from these ions.

Mg II. Mg II is found only in the spectrum of SN $2000 \mathrm{dk}$ at $+5 \mathrm{~d}$ which is the oldest spectrum in the sample to show strong $\mathrm{Mg}$ II absorption. $\mathrm{Mg}$ II is the primary contributor forming the distinctive notch near $1.60 \mu \mathrm{m}$. This spectrum also has strong Mg II absorption from the 0.9227 and $1.0927 \mu \mathrm{m}$ lines. The $1.0092 \mu \mathrm{m} \mathrm{Mg}$ II line is expected to be 2 orders of magnitude weaker than $1.0927 \mu \mathrm{m}$ but at $+5 \mathrm{~d}$ or earlier it may contribute to the absorption near $0.97 \mu \mathrm{m}$ either alone or in a blend with Fe II.

Si I. Si I is not detected in our data although this ion produces many strong lines in the NIR including lines at 1.2032, 1.0827, and $1.0585 \mu \mathrm{m}$. These lines may help form the features found near 1.03 and $1.06 \mu \mathrm{m}$ in the oldest spectrum from this group (SN 2004da at $+24 d$ ).

Si II. Si II is possible with the $0.9413 \mu \mathrm{m}$ line contributing to the $0.92 \mu \mathrm{m}$ absorption found in some spectra in this group. The Si II $1.6930 \mu \mathrm{m}$ line may also be part of the $1.60 \mu \mathrm{m}$ feature. The $1.1737 \mu \mathrm{m}$ line from Si II is not detected so it is unlikely that the $1.1311 \mu \mathrm{m}$ line is responsible for the feature found near $1.12 \mu \mathrm{m}$ in some of the spectra. The $1.12 \mu \mathrm{m}$ feature does not occur in the same spectra as the $0.92 \mu \mathrm{m}$ feature which eliminates the possible association of the $1.12 \mu \mathrm{m}$ feature with Si II.

S I. S I at $0.9223 \mu \mathrm{m}$ contributes to the $0.90 \mu \mathrm{m}$ feature in all spectra in this group and probably replaces $\mathrm{Mg}$ II as the primary source of the absorption after $+5 \mathrm{~d}$ post-maximum. Velocities for this ion begin at $10,300-13,700 \mathrm{~km} \mathrm{~s}^{-1}$ at $+0 \mathrm{~d}$ and diminish to 8900 at $+18 \mathrm{~d}$. After +10 days, some spectra do not have any absorption feature at this location. The $1.3809 \mu \mathrm{m} \mathrm{S}$ I line is too far into the region of high atmospheric opacity to be detected. Other possible associations can be made for $\mathrm{S}_{\mathrm{I}}$ at 0.9423 and $1.1349 \mu \mathrm{m}$, but they both have serious questions. The feature found near $1.10 \mu \mathrm{m}$ is possibly associated with the $1.1349 \mu \mathrm{m}$ line and the velocity shift is plausible of 8500 at
$+5 \mathrm{~d}$ and 7000 at $+18 \mathrm{~d}$. However, this feature is near an order transition in SXD spectra which casts suspicion on any features at this location from SXD spectra (in particular, the spectrum from SNe 2000dk (+5d)). Since LRS spectra do not have order transitions, the weak feature near $1.1 \mu \mathrm{m}$ is more likely to be real in the LRS spectra. The S I $0.9423 \mu \mathrm{m}$ line may also produce features in a few spectra, but the $0.92 \mu \mathrm{m}$ absorption feature does not appear in the same spectra that produce other probable S I identifications.

Ca II IR triplet. The Ca II IR triplet velocities remain well above $10,000 \mathrm{~km} \mathrm{~s}^{-1}$ for the spectra in this group. Separate features can be detected in the spectra from this group for both the $0.8358 \mu \mathrm{m}$ blend and the $0.8662 \mu \mathrm{m}$ line as described in the previous appendix. The shape of the absorption feature is consistent with estimates that the $0.8538 \mu \mathrm{m}$ blend is about twice as strong as the $0.8662 \mu \mathrm{m}$ line.

Other Ca II. Other Ca II is not detected from the lines at 1.1876 or $0.9906 \mu \mathrm{m}$ or from other Ca II line. The weak features found in some spectra near $1.10 \mu \mathrm{m}$ are possibly associated with the $1.1876 \mu \mathrm{m}$ line but the velocity would be more than $21,000 \mathrm{~km} \mathrm{~s}^{-1}$, which is inconsistent with other photospheric velocities during this phase. That makes it unlikely that the Ca II line at $0.9931 \mu \mathrm{m}$, which is estimated to be $\sim 20$ times weaker, provides a contribution to the absorption found near $0.97 \mu \mathrm{m}$.

Mn II. Mn II at $0.9447 \mu \mathrm{m}$ is a possible detection in the spectra from this group that contain features near $0.92 \mu \mathrm{m}$. This feature may be attributed to either $\mathrm{Mn}$ II or Si II alone or in a blend of both. A rest wavelength of $0.9447 \mu \mathrm{m}$ would produce velocities of $8500-12,500 \mathrm{~km} \mathrm{~s}^{-1}$ for Mn II, or about $1000 \mathrm{~km} \mathrm{~s}^{-1}$ greater than velocities listed for Si II at $0.9413 \mu \mathrm{m}$ in Table 4. However, Mn II produces more than 90 strong lines between 0.9309$0.9509 \mu \mathrm{m}$ so it is not possible to measure a definitive velocity. These lines do not appear in Tables 5 or 6 because they are slightly weaker than the $0.9447,0.9388$, and $0.9486 \mu \mathrm{m}$ lines that do appear in the tables. The strongest Mn II lines at 0.7414 and $0.7342 \mu \mathrm{m}$ may form the features found with a similar velocity separation near $0.72 \mu \mathrm{m}$, but there are only two spectra with which to compare these lines. An alternative identification for those features is $\mathrm{Fe}$ II.

Steep Increase in Flux Found Near $1.55 \mu \mathrm{m}$. The steep increase in flux found near $1.55 \mu \mathrm{m}$ may define the transition from partial to complete silicon burning. This feature is present in all spectra from this group but it is less well defined in the spectra from SNe 2005am (+14d) and 2004da (+24d). This does not appear to be an evolutionary effect because the features are present in many later spectra.

Fe II. Fe II at $0.9998 \mu \mathrm{m}$ is the likely source of the absorption found near $0.97 \mu \mathrm{m}$ in all spectra from this group through $+18 \mathrm{~d}$. Before $+5 \mathrm{~d}$ (see the previous appendix) this feature may receive a contribution from $\mathrm{Mg}$ II at $1.0092 \mu \mathrm{m}$, but the absorption is strongest from $+5 \mathrm{~d}$ to $+10 \mathrm{~d}$ when it is likely to be entirely due to Fe II. Velocities for Fe II measured from the $0.9998 \mu \mathrm{m}$ line are near $9000 \mathrm{~km} \mathrm{~s}^{-1}$ at $+0 \mathrm{~d}$ and diminish to less than 3000 by $+18 \mathrm{~d}$. Fe II is also likely to be the source of absorptions near 0.75 and $0.72 \mu \mathrm{m}$ after $+10 \mathrm{~d}$ from the two strong lines at 0.7712 and $0.7462 \mu \mathrm{m}$ at about $8500 \mathrm{~km} \mathrm{~s}^{-1}$ in the spectra from SNe 2004bk (+12) and 2002ef $(+16)$. The Fe II line from $0.9998 \mu \mathrm{m}$ appears to diminish in strength after about $+10 \mathrm{~d}$ but it becomes strong again about 1 month postmaximum. 
Co II. Co II forms distinct features in the later spectra in this group. Spectra obtained at $+10 \mathrm{~d}$ or later show distinct absorption at the location of many Co II lines, most prominently: 1.7772, $1.7462,2.2205,2.4596$, and $2.3613 \mu \mathrm{m}$ at velocities near $9000 \mathrm{~km} \mathrm{~s}^{-1}$ (see Figure 17). Possible identification of Co II lines from 1.7772 and $1.7462 \mu \mathrm{m}$ can be made in the spectrum from SN $2000 \mathrm{dk}$ as early as $+5 \mathrm{~d}$.

\section{A.4. Transitional Phase (Inner Layers; $+37 d$ to $+75 d$ with Respect to $V_{\max }$ )}

This epoch is a transitional phase between the photospheric era and a true nebular phase. Discussion in this appendix makes reference to Figure 16 showing seven spectra from $\mathrm{SNe}$ Ia obtained between $+37 \mathrm{~d}$ and $+75 \mathrm{~d}$ with respect to $V_{\max }$ and Figure 17 which includes 13 spectra from our sample and two additional spectra from other sources. In Figure 17, the positions of strong $\mathrm{Co}$ II and Fe II lines are marked so that they correspond to $11,000 \mathrm{~km} \mathrm{~s}^{-1}$ at the top spectrum and $6000 \mathrm{~km} \mathrm{~s}^{-1}$ at the bottom spectrum. This reflects to the reduction in measured velocities for these spectra. The data plotted in yellow ( $\mathrm{SNe}$ 1998aq and 1998bu) were obtained by R. J. Rudy \& R. Puetter (2007, private communication) and have not been previously published. Co II lines are easily detected in absorption as early as 2 weeks post-maximum and Fe II lines (in addition to the $0.9998 \mu \mathrm{m}$ which appears as early as $+0 \mathrm{~d}$ ) begin to appear about 1 month post-maximum (see Sections 3.1.3 and 3.1.4).

This group displays a lot of new structure relative to earlier NIR spectra and the features are remarkably similar from one spectrum to the next. The spectrum of SN 2004bv at $+60 \mathrm{~d}$ fits well with the others in this group although the spectrum from this $\mathrm{SN}$ at $-5 \mathrm{~d}$ was unusual (see Figure 12). This is the same time frame in which unreddened SNe Ia have a very low dispersion for $B-V$ colors (Lira 1996). The common assumption about this era is that expansion has reduced the opacity beyond the point where a distinct photosphere can be defined and individual absorption features can no longer be produced. Recent work by Branch et al. (2008) suggests spectra from this epoch can be modeled by absorption features from permitted lines.

Ca II IR triplet. The Ca II IR triplet velocities remain near $10,000 \mathrm{~km} \mathrm{~s}^{-1}$ which is consistent with this feature being produced by $\mathrm{Ca}$ II that was not synthesized in the explosion (see Section 5.3). Separate features can be detected in many spectra from this group for both the $0.8358 \mu \mathrm{m}$ blend and the $0.8662 \mu \mathrm{m}$ line.

Steep Increase in Flux Found Near $1.55 \mu \mathrm{m}$. The steep increase in flux found near $1.55 \mu \mathrm{m}$ may define the transition from partial to complete silicon burning. This feature is present is all spectra from this group but is more sharply defined in some spectra compared to others. As shown in Figure 16, the spectrum from SN 2004bv at +60 displays this feature but it is smeared out in the spectrum of SN $2001 \mathrm{fe}$, also obtained at $+60 \mathrm{~d}$.

Fe II. Fe II lines at 0.7712, 0.9998, and $1.0863 \mu \mathrm{m}$ appear in absorption beginning about 30 days after maximum light and in all spectra in this group (Figure 17). The Fe II $0.9998 \mu \mathrm{m}$ line is present is spectra from about $+0 \mathrm{~d}$ to $+18 \mathrm{~d}$, it is not detected from $+18 \mathrm{~d}$ to $+24 \mathrm{~d}$, and then reappears after $+30 \mathrm{~d}$ (see Figure 10 ). No other detections from individual Fe II lines are reliable from NIR spectra prior to this later phase.

Co II. Co II lines are clearly detected in wavelength regions where a pseudophotosphere is created at extended radii (1.5-
1.85 and 2.05-2.45 $\mu \mathrm{m}$; see Section 5.3) and Figure 17. Strong absorption features are detected from many Co II lines. The LRS spectra from SNe 2003cd (+45) and 2004bv (+60) as shown in Figures 16 only marginally resolve Co II absorptions for 1.7772 , $1.7462,2.2205,2.4596$, and 2.3613 lines and the group of Co II lines from 1.57 to 1.67 are not resolved at all. Figure 17 shows how the locations of these lines shift to longer wavelengths with increasing time of observation relative to maximum light. The positions of strong Co II and Fe II lines are drawn in the figure so that the indicated velocities are $11,000 \mathrm{~km} \mathrm{~s}^{-1}$ at the top spectrum and $6000 \mathrm{~km} \mathrm{~s}^{-1}$ at the bottom spectrum.

\section{A.5. Summary of Line Identification by Ion for the Entire Sample}

Velocity references are compiled in Table 4 and Figure 19.

He I. He I is not detected from either of the strong lines at 1.0830 or $2.0581 \mu \mathrm{m}$. Unrealistically low Doppler velocities are required to align the $1.0830 \mu \mathrm{m}$ line with the absorption feature found near $1.05 \mu \mathrm{m}$. The 2.0581 line (which is expected to be 612 times weaker than the 1.083 line depending on temperature), is not observed.

C I. C I is not detected. Speculative identifications can be made for lines at $0.9406,1.1331,1.1756$, and $1.6890 \mu \mathrm{m}$ in the very early spectra, but if these lines produce absorption features, the much stronger lines at 1.0693 and 0.9087 should also be detected and they are not. At each location of possible $\mathrm{C}$ I detection there are plausible alternative identifications (see Figure 20).

O I. O I forms a strong feature from the $0.7773 \mu \mathrm{m}$ line producing an absorption near $0.75 \mu \mathrm{m}$. This feature is found in all LRS spectra up to $+10 \mathrm{~d}$ and possibly as late as $+18 \mathrm{~d}$ (SXD spectra lack the wavelength coverage to include this feature). $\mathrm{O}_{\mathrm{I}}$ is also likely as a contributor to the 0.89 feature from the line at $0.9264 \mu \mathrm{m}$. Doppler velocities are $9300-15,700 \mathrm{~km} \mathrm{~s}^{-1}$ with most measurements near $11,000 \mathrm{~km} \mathrm{~s}^{-1}$.

$\mathrm{C}_{\text {II }}$ and $\mathrm{O}_{\text {II. }} \mathrm{C}_{\text {II }}$ and $\mathrm{O}_{\text {II }}$ are not detected. All $\mathrm{C}_{\text {II }}$ lines in the NIR are factors of $10^{2}-10^{4}$ weaker than the $C_{\text {II }}$ at $0.6580 \mu \mathrm{m}$ line which has been tentatively identified in a few optical spectra.

Mg II. Mg II is found in all spectra between $-14 \mathrm{~d}$ and $+5 \mathrm{~d}$, producing strong features from lines at 0.9227 and $1.0927 \mu \mathrm{m}$. Velocities are 9800-14,700 $\mathrm{km} \mathrm{s}^{-1}$ with a few anomalies near $9000 \mathrm{~km} \mathrm{~s}^{-1}$. Measured velocities for the 0.9227 line are $\approx 1000 \mathrm{~km} \mathrm{~s}^{-1}$ slower than for the $1.0927 \mu \mathrm{m}$ line as a result of the enormous $\mathrm{P}$ Cygni emission from Ca II impinging on the absorption feature. Many spectra also contain features from weaker Mg II lines at 1.0092 and $1.6787 \mu \mathrm{m}$.

Si I. Si I is not detected although some spectra exhibit weak features that could possibly be attributed to this ion from lines at $1.2032,1.0827$, or $1.0585 \mu \mathrm{m}$.

Si II. Si II from the $0.9413 \mu \mathrm{m}$ line produces the feature near $0.91 \mu \mathrm{m}$ at velocities from 10,200 to $14,100 \mathrm{~km} \mathrm{~s}^{-1}$ between $-14 \mathrm{~d}$ and $+5 \mathrm{~d}$. After $+5 \mathrm{~d}$ the feature appears intermittently as late as $+18 \mathrm{~d}$, but this may be a blend with Mn II or entirely due to Mn II. The Si II 1.6930 line contributes to the 1.60 feature. The 1.1737 line is not detected so it is unlikely that the 1.1311 line is responsible for the feature found near 1.12 in some of the spectra. The 1.12 feature does not occur in the same spectra as the 0.91 feature so the Si II identification is unlikely.

Si III. Si III is possible in the earliest spectra from the $0.9324 \mu \mathrm{m}$ line at the very low velocity of $8500 \mathrm{~km} \mathrm{~s}^{-1}$. Other possible 
identifications for this ion are the feature at 1.22 (from 1.2541, a blend of 1.2523 and 1.2601, at $9600 \mathrm{~km} \mathrm{~s}^{-1}$ ) and 1.09 (from 1.1341 at $10,500 \mathrm{~km} \mathrm{~s}^{-1}$ ). Si III can be present in the same spectrum with $\mathrm{Si}$ II if the photosphere is deep within the envelope so that temperature and pressure differences between absorbing regions near the photosphere and in the outer layers contain different ionizations of the same element (Section 5.3).

S I. S I at $0.9223 \mu \mathrm{m}$ replaces Mg II as the primary source of the 0.90 feature after maximum light. Velocities begin at 10,300 $13,700 \mathrm{~km} \mathrm{~s}^{-1}$ at $+0 \mathrm{~d}$ and diminish to 8900 at $+18 \mathrm{~d}$. After +10 days, there are a few spectra with no feature at this location. Other possible associations can be made for $\mathrm{S}_{\mathrm{I}}$ at 1.3809 , 0.9423 , and 1.1349 , but they all have serious questions.

Ca II IR triplet. Ca II IR triplet is the strongest feature in most spectra. Velocities are greater than $10,000 \mathrm{~km} \mathrm{~s}^{-1}$ for most spectra, although there are exceptions such as SN $2001 \mathrm{bf}(+4 \mathrm{~d})$ for which the velocity is $7600 \mathrm{~km} \mathrm{~s}^{-1}$ and this feature is very weak in the spectrum from SN 2004bv at -5 d. SN 2001bf has very low velocities for all identified lines and SN 2004bv may be 91T-like. Distinct individual features can be detected in most of the SXD spectra for both the 0.8358 blend and the 0.8662 line. The shape of the absorption feature is consistent with estimates that the 0.8538 blend is $\sim$ twice as strong as the 0.8662 line. Very low excitation values for these lines suggest that absorption features from the Ca II IR triplet may not come from freshly synthesized material.

High-Velocity Ca II. All LRS spectra obtained at $-5 \mathrm{~d}$ or earlier appear to have high-velocity Ca II components with velocities measured between 18,800 and $24,000 \mathrm{~km} \mathrm{~s}^{-1}$.

Other Ca II. The other CaII is not detected from the next strongest lines at 1.1876 or $0.9906 \mu \mathrm{m}$ or from other Ca II lines in any spectrum in our sample. Ca II appears to be absent from the freshly synthesized explosion products.

Mn II. Mn II at $0.9447 \mu \mathrm{m}$ is possibly detected after maximum light. The strongest Mn II lines at 0.7414 and $0.7342 \mu \mathrm{m}$ form features at similar velocities near $0.72 \mu \mathrm{m}$, but there are only two spectra with which to compare this line.

Fe II. Fe II is detected in post-maximum spectra from the $0.9998 \mu \mathrm{m}$ line at velocities near $9500 \mathrm{~km} \mathrm{~s}^{-1}$ at $+0 \mathrm{~d}$, diminishing to less than 3000 by $+18 \mathrm{~d}$. This line may contribute to the feature in pre-maximum spectra attributed to the $\mathrm{Mg}$ II line at $1.0092 \mu \mathrm{m}$. Fe II is likely to be the source of absorptions near 0.75 and 0.72 after $+10 \mathrm{~d}$. Fe lines are more difficult to compare based on our simple line-strength estimates because the actual line strengths are affected by multiple transitions. As a consequence, the simple calculations in our tables for the relative estimated strength of each $\mathrm{Fe}$ II line may be less accurate than the estimates for smaller atoms. In spectra from our sample obtained before $+37 \mathrm{~d}$, the $0.9998 \mu \mathrm{m}$ line is the only Fe II line detected, although the calculated line strengths suggest that lines at $0.7462,0.7712,1.0500$, and $1.0863 \mu \mathrm{m}$ lines should also be detected.

Co II. Co II may be detected soon after maximum light in some of the high-resolution spectra. After two weeks post-maximum, Co II lines are clearly detected at velocities beginning near $10,000 \mathrm{~km} \mathrm{~s}^{-1}$ and diminishing to $6000 \mathrm{~km} \mathrm{~s}^{-1}$. Co II is found in wavelength regions where a pseudophotosphere is created at extended radii (1.5-1.85 and 2.05-2.45 $\mu \mathrm{m})$. Co II is most obvious in the higher resolution (SXD) spectra and individual Co II lines are marginally resolved in many other later time spectra.

\section{REFERENCES}

Abia, C., Domínguez, I., Höflich, P., \& Straniero, O. 2001, ApJ, 557, 126 Branch, D., et al. 2008, PASP, 120, 135

Conley, A., et al. 2008, ApJ, 681, 482

Cushing, M. C., Vacca, W. D., \& Rayner, J. T. 2004, PASP, 116, 362

Gamezo, V. N., Khokhlov, A. M., Oran, E. S., Chtchelkanova, A. Y., \& Rosenberg, R. O. 2003, Science, 299, 77

Garnavich, P., et al. 1998, ApJ, 493, 53

Guy, J., et al. 2007, A\&A, 466, 11

Hamuy, M., Phillips, M. M., Suntzeff, N. B., Schommer, R. A., Maza, J., \& Aviles, A. 1996, AJ, 112, 2398

Hamuy, M., et al. 2002, AJ, 124, 417

Höflich, P. 1995, ApJ, 443, 89

Höflich, P., Gerardy, C., Fesen, R., \& Sakai, S. 2002, ApJ, 568, 791

Höflich, P., Mueller, E., \& Khokhlov, A. 1993, A\&A, 268, 570

Höflich, P., Wheeler, J. C., \& Thielemann, F. K. 1998, ApJ, 495, 617

Hoyle, P., \& Fowler, W. A. 1960, ApJ, I132, 565

Jha, S., Riess, A., \& Kirshner, R. 2007, ApJ, 659, 122

Khokhlov, A. M. 1991, A\&A, 245, 114

Khokhlov, A. M., Oran, E. S., \& Wheeler, J. C. 1997, ApJ, 478, 678

Kowalski, M., et al. 2008, ApJ, 686, 749

Lira, P. 1996, MS thesis, Univ. Chile

Marion, G. H., Höflich, P., Gerardy, C. L., Vacca, W. D., Wheeler, J. C., \& Robinson, E. L. 2006, ApJ, 645, 1392

Marion, G. H., Höflich, P., Vacca, W. D., \& Wheeler, J. C. 2003, ApJ, 591, 316

Meikle, W. P. S., et al. 1996, MNRAS, 281, 263

Perlmutter, S., et al. 1999, ApJ, 517, 565

Phillips, M. M. 1993, ApJ, 413, 105

Rayner, J. T., Toomey, D. W., Onaka, P. M., Denault, A. J., Stahlberger, W. E., Vacca, W. D., Cushing, M. C., \& Wang, S. 2003, PASP, 115, 362

Riess, A. G., Press, W. H., \& Kirshner, R. P. 1995, ApJ, 438, L17

Riess, A. G., et al. 1998, AJ, 116, 1009

Schmidt, B., et al. 1998, ApJ, 507, 46

SUSPECT 2008, Online Supernovae Spectrum Archive, http://bruford.nhn.ou.edu/ $\sim$ suspect/index.html

Tanaka, M., et al. 2008, ApJ, 677, 448

Vacca, W. D., Cushing, M. C., \& Rayner, J. T. 2003, PASP, 115, 389

Wang, L., Höflich, P., \& Wheeler, J. C. 1997, ApJ, 483, L29

Wang, L., \& Wheeler, J. C. 2008, ARA\&A, 46, 433

Wang, L., et al. 2003, ApJ, 591, 1110

Weller, J., \& Albrecht, A. 2002, Phys. Rev. D, 65, 103512

Wheeler, J. C., Höflich, P., Harkness, R. P., \& Spyromilio, J. 1998, ApJ, 496, 908

Yoon, S.-C., \& Langer, N. 2004a, A\&A, 419, 623

Yoon, S.-C., \& Langer, N. 2004b, A\&A, 419, 645 